\title{
Fe-Ti-V-P ore deposits associated with Proterozoic massif-type anorthosites and related rocks
}

\author{
Bernard Charlier $^{\mathrm{a}, \mathrm{b}, *}$, Olivier Namur $^{\mathrm{a}}$, Olivier Bolle ${ }^{\mathrm{b}}$, Rais Latypov ${ }^{\mathrm{c}}$, Jean-Clair Duchesne ${ }^{\mathrm{b}}$ \\ ${ }^{a}$ Institut für Mineralogie, Leibniz Universität Hannover, 30167 Hannover, Germany \\ ${ }^{b}$ Department of Geology, University of Liège, 4000 Sart Tilman, Belgium \\ c School of Geosciences, University of Witwatersrand, PO Wits 2050, South Africa
}

\section{A R T I C L E I N F O}

\section{Article history:}

Received 18 June 2014

Accepted 11 November 2014

Available online 21 November 2014

\section{Keywords:}

Titanium ore

Nelsonite

Ilmenite

Tellnes

Lac Tio

Damiao

\begin{abstract}
A B S T R A C T
Magmatic rocks containing economic concentrations of iron, titanium, vanadium and phosphorous are commonly associated with massif-type anorthosites and related rocks. This rock association is part of the anorthositemangerite-charnockite-(rapakivi-)granite suites that are restricted to the Proterozoic. Understanding the geochemistry and emplacement mechanisms of ilmenite, magnetite and apatite ore deposits is crucial for exploration, efficient mining operations and ore processing. This review discusses the controlling factors on the grade of an ore, its mineralogy, and its major and trace element distribution. We present petrogenetic models of currently mined deposits (Lac Tio, Tellnes, Damiao) and discuss the characteristics of minor ore bodies from anorthosite provinces worldwide (Grenville, North China Craton, East European Craton, Rogaland, Laramie). Models of formation of anorthosite and related rocks are presented, as well as the nature of the possible parental magmas of the suite. A mineralogical classification of Fe-Ti ores is proposed: (1) Gabbro-noritic ilmenite ore \pm apatite \pm magnetite; (2) Ti-magnetite-dominated ore; (3) Nelsonite (Fe-Ti oxides + apatite); and (4) Rutile-ilmenite ore. The stability of ilmenite and magnetite is then critically reviewed and the influence of various factors, particularly oxygen fugacity and crystallization pressure, is examined. We discuss liquidus compositions of Fe-Ti oxides and the behavior of important trace elements such as $\mathrm{Cr}$ and V, both of which are sensitive to $\mathrm{fO}_{2}$ variations. Postcumulus evolution of both oxides can occur due to re-equilibration with trapped liquid, re-equilibration with ferromagnesian silicates, exsolution, oxidation, reaction between ilmenite and magnetite, and metamorphic overprinting. These various processes are described and their effects on the oxide geochemistry are emphasized. Several potential ore-forming processes have been invoked and can explain the formation of huge concentration of ilmenite, \pm magnetite, \pm apatite. Fractional crystallization can be combined with crystal sorting and plagioclase buoyancy to produce relative enrichment of dense ore minerals. Silicate liquid immiscibility can segregate conjugate Si-rich and Fe-rich melts, the latter being enriched in Fe-Ti-P. Magma mixing can produce hybrid magmas located in a single-phase field of the phase diagram and precipitate a pure ilmenite cumulate. Alternative processes are also described, such as ejection of Fe-Ti-enriched residual melts by filter-pressing and compaction, solidstate remobilization of ilmenite in veins, and hydrothermal transport of Fe and Ti from the host anorthosite followed by concentration in veins and lenticular ore bodies. The magnetic properties of Fe-Ti ore deposits present contrasting signatures, depending on whether the natural remanent magnetization is dominated by hemo-ilmenite or multi-domain magnetite. Micro- and macro-scale deformation features of ore rocks are intimately correlated with magma emplacement, and with ballooning of the anorthosite diapir associated with gravitational sagging of dense ore bodies. Exploration perspectives show that oxide-apatite gabbronorites are interesting targets because ilmenite in these rocks is poorer in $\mathrm{Cr}$ and $\mathrm{Mg}$, and because the Ti-resource may be combined with apatite and vanadiferous magnetite.
\end{abstract}

(c) 2014 Elsevier B.V. All rights reserved.

\section{Contents}

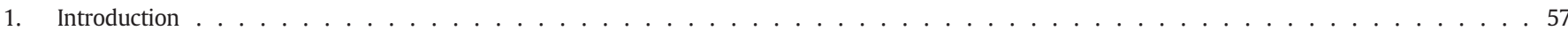

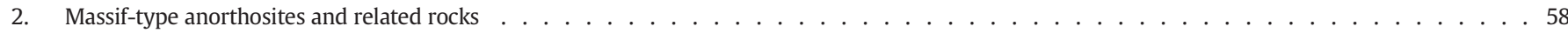

\footnotetext{
* Corresponding author at: Department of Geology, University of Liège, 4000 Sart Tilman, Belgium. Tel.: + 3243662250.

E-mail address: b.charlier@ulg.ac.be (B. Charlier).
} 
2.1. Spatial and temporal distribution . . . . . . . . . . . . . . . . . . . . . . . . . . . . . . . . . . . . 58

2.2. Crystallization conditions of anorthosites . . . . . . . . . . . . . . . . . . . . . . . . . . . . . . . . . . . . . . . . 59

2.3. Composition and source for parental magmas: mantle vs. lower crust . . . . . . . . . . . . . . . . . . . . . . . . . . . . . . . . . 60

2.4. Related rocks: comagmatic or simply coeval with massif-type anorthosites? . . . . . . . . . . . . . . . . . . . . . . . . . . . . . 60

3. Characteristics of major deposits . . . . . . . . . . . . . . . . . . . . . . . . . . . . . . . . . . . . . . . . . . . . 61

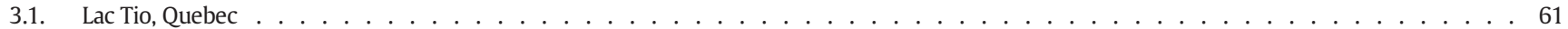

3.2. Tellnes, SW Norway . . . . . . . . . . . . . . . . . . . . . . . . . . . . . . . . . . . . . . . . . . 61

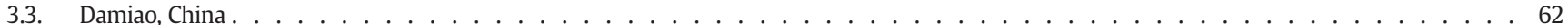

4. Mineralization styles, grades and sizes . . . . . . . . . . . . . . . . . . . . . . . . . . . . . . . . . . . . . . . . . . 62

4.1. Massive deposits .. . . . . . . . . . . . . . . . . . . . . . . . . . . . . . . . . . . . 62

4.2. Stratiform deposits . . . . . . . . . . . . . . . . . . . . . . . . . . . . . . . . . . . . . . . . . . . 62

4.3. Massive to layered tabular bodies . . . . . . . . . . . . . . . . . . . . . . . . . . . . . . . 65

4.4. Lenticular ore bodies . . . . . . . . . . . . . . . . . . . . . . . . . . . . . . . . . . . . . . . . . . . . . . . . . . . . 65

5. Sequence of crystallization in layered intrusions . . . . . . . . . . . . . . . . . . . . . . . . . . . . . . . . . . . . . . . . . . . 65

6. A mineralogical classification for Fe-Ti-V-P deposits . . . . . . . . . . . . . . . . . . . . . . . . . . . . . . . . . 66

6.1. (Gabbro-)noritic ilmenite ore \pm apatite \pm magnetite . . . . . . . . . . . . . . . . . . . . . . . . . . . . . . . . . . 67

6.2. Ti-magnetite- \pm apatite-dominated ore . . . . . . . . . . . . . . . . . . . . . . . . . . . . . . . . . . . . . 67

6.3. Nelsonite . . . . . . . . . . . . . . . . . . . . . . . . . . . . . . . . . . . . . . . . . . 68

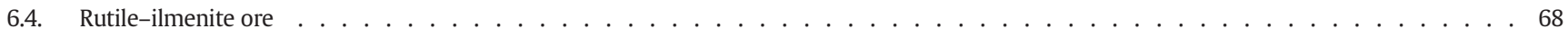

7. The stability of ilmenite and magnetite . . . . . . . . . . . . . . . . . . . . . . . . . . . . . . . . . . . . . . . . . 68

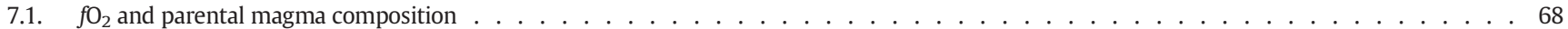

7.2. The role of polybaric crystallization . . . . . . . . . . . . . . . . . . . . . . . . . . . . . . . . . . . . . . 69

7.3. The role of $\mathrm{P}_{2} \mathrm{O}_{5}$. . . . . . . . . . . . . . . . . . . . . . . . . . . . . . . . . . . . . . . . . 69

8. Liquidus compositions of Fe-Ti oxides . . . . . . . . . . . . . . . . . . . . . . . . . . . . . . . . . . . . . . . . . . . . . 69

8.1. Major elements . . . . . . . . . . . . . . . . . . . . . . . . . . . . . . . . . . . . . 69

8.2. Cr and V partitioning. . . . . . . . . . . . . . . . . . . . . . . . . . . . . . . . . . . . . . . . . 69

9. Postcumulus evolution of Fe-Ti oxide minerals . . . . . . . . . . . . . . . . . . . . . . . . . . . . . . . . . . . . . . . . . . . . . 70

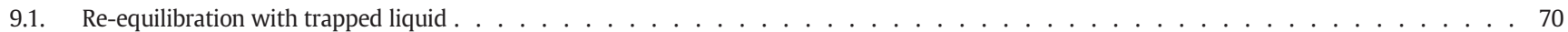

9.2. Re-equilibration with ferromagnesian silicates . . . . . . . . . . . . . . . . . . . . . . . . . . . . . . . . . . . . . . . . . 70

9.3. Exsolution, oxy-exsolution, oxidation . . . . . . . . . . . . . . . . . . . . . . . . . . . . . . . . . . . . . . . . . 71

9.4. Reaction between ilmenite and magnetite . . . . . . . . . . . . . . . . . . . . . . . . . . . . . . . . . . . . . . . . . 73

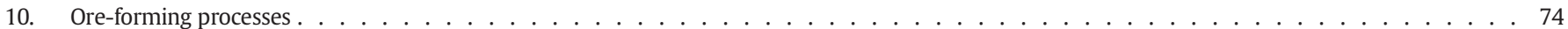

10.1. Fractional crystallization, crystal sorting and plagioclase buoyancy . . . . . . . . . . . . . . . . . . . . . . . . . . . . . . . . 74

10.2. Immiscibility . . . . . . . . . . . . . . . . . . . . . . . . . . . . . . . . . . . . 74

10.3. Magma mixing . . . . . . . . . . . . . . . . . . . . . . . . . . . . . . . . . . . . . . . 74

10.4. Filter-press compaction . . . . . . . . . . . . . . . . . . . . . . . . . . . . . . . . . . . . . . 74

10.5. Solid-state remobilization . . . . . . . . . . . . . . . . . . . . . . . . . . . . . . . . . . . . . . . . . . . . . . . 75

10.6. Hydrothermal remobilization . . . . . . . . . . . . . . . . . . . . . . . . . . . . . . . . . . . . 75

11. Metamorphism: a natural beneficiation processes? . . . . . . . . . . . . . . . . . . . . . . . . . . . . . . . . . . . . 75

12. Magnetic signatures of Fe-Ti-V-P deposits . . . . . . . . . . . . . . . . . . . . . . . . . . . . . . . . . . . . . . 75

13. Structural analysis and deformation . . . . . . . . . . . . . . . . . . . . . . . . . . . . . . . . . . . . 76

14. Conclusions . . . . . . . . . . . . . . . . . . . . . . . . . . . . . . . . . . . . . . . . . . . . . . . 77

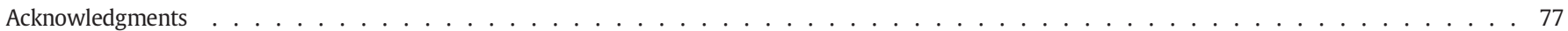

References . . . . . . . . . . . . . . . . . . . . . . . . . . . . . . . . . . . . . . . 77

\section{Introduction}

Ilmenite and rutile are the main sources of titanium with $90 \%$ of commercial $\mathrm{TiO}_{2}$ coming from ilmenite and $10 \%$ from rutile. Hardrock deposits associated with Proterozoic anorthosites provide $30 \%$ of ilmenite while the remaining $70 \%$, and all rutile, comes from placers (Gambogi, 2010). Despite an abundance of world resource for Ti ores, the industry continues to prospect for better quality ilmenite, principally because of processing costs and waste disposal problems associated with the high iron and trace-element contents of ilmenite. The modern chloride process is far less polluting than the older sulfate process because it does not produce waste iron sulfate (Chernet, 1999). Both processes have restrictions on the contents of chromophore elements such as $\mathrm{Cr}$ and $\mathrm{Mn}$, but the chloride process also has much stricter limits on concentrations of the alkali elements, especially $\mathrm{Ca}$ and $\mathrm{Mg}$, as well as on grain-size. Moreover, the chloride process works best with highTi low-Fe ores such as rutile or leucoxene from heavy mineral beach sands.

Three hard rock deposits are currently mined: Lac Tio (Quebec; Charlier et al., 2010b), Tellnes (SW Norway; Charlier et al., 2007), and Damiao (China; Chen et al., 2013). In these mines it is becoming essential to plan ore mixing from different locations in the pit to obtain ilmenite concentrates with a constant composition. It is therefore important to understand compositional variations inside these ore bodies, which a growing database is now documenting.

As a result of mineral processing constraints, oxide-apatite gabbronorites are becoming new targets, partly because the recovery of Ti ore may be combined with that of phosphorous in apatite and vanadium in magnetite, but mostly because ilmenite in these rocks commonly has significantly less $\mathrm{Cr}$ and $\mathrm{Mg}$ than ilmenite from other mines. Examples of these types of deposits associated with anorthositemangerite-charnockite-(rapakivi-)granite (AMCG) suites include the Grader (Charlier et al., 2008), Bjerkreim-Sokndal (Duchesne, 1972; Meyer et al., 2002) and Fedorivka (Duchesne et al., 2006) layered intrusions.

The igneous origin of Fe-Ti deposits is widely accepted, but there are many magmatic processes that produce these oxide concentrations. Major processes commonly invoked are as follows: segregation of an immiscible $\mathrm{Fe}-\mathrm{Ti}-\mathrm{P})$-rich magma; fractional crystallization with oxide settling (possibly accompanied by plagioclase flotation); magma mixing; polybaric crystallization; and solid-state remobilization. These ore-forming processes are not mutually exclusive, and several mechanisms can concentrate ilmenite \pm magnetite \pm apatite during cooling of the parental magma. 
Ti minerals encountered in these deposits are mainly members of two solid solution series: the hematite-ilmenite series $\left(\mathrm{Fe}_{2} \mathrm{O}_{3}-\mathrm{FeTiO}_{3}\right)$ and the magnetite-ulvöspinel-Al-spinel series $\left(\mathrm{Fe}_{3} \mathrm{O}_{4}-\mathrm{Fe}_{2} \mathrm{TiO}_{4}\right.$ $(\mathrm{Fe}, \mathrm{Mg}) \mathrm{Al}_{2} \mathrm{O}_{4}$ ). Rutile is rare. Lenses of Al-spinel in ilmenite and external granule exsolutions are evidence that a small amount of Al-spinel can dissolve in hemo-ilmenite. Since Buddington and lindsley (1964), many experimental studies have clearly shown that the primary compositions of coexisting Fe-Ti oxides depend on temperature and oxygen fugacity. Another factor, the $\mathrm{TiO}_{2}$ content of parental melts, may control the stability of (hemo-)ilmenite and (Ti-)magnetite (Toplis and Carroll, 1995). This is critical for mining considerations, since Ti in magnetite has a low value and a limited market.

Controlling factors on the grade of the ore, the mineralogy and composition of associated minerals, and the distribution of major and trace elements in Fe-Ti oxides are highly variable. The chemistry of the magnetite-ulvöspinel-spinel and hematite-ilmenite series is moreover complicated by various subsolidus processes, mainly exsolution and oxidation, which can drastically modify the primary compositions of high-temperature liquidus phases. The concentration of some polluting elements, particularly $\mathrm{Cr}$ and $\mathrm{Mg}$, which affect industrial ilmenite processing, thus depends on many processes.

In this study, we present a review of typical $\mathrm{Fe}-\mathrm{Ti} \pm \mathrm{V} \pm \mathrm{P}$-rich rocks in several anorthosite provinces. The ore deposits display particular characteristics concerning ore composition and grade, associated minerals and deposit morphology. These case studies thus provide a wide-ranging overview of Fe-Ti ore-forming processes and the factors controlling ore composition. Particular attention is given to their relation to their host rocks, commonly anorthosite plutons or layered intrusions. Liquidus compositions and postcumulus evolution of oxides are also discussed. The objective is to consider the range of ore-forming processes in Proterozoic anorthosites and thereby extract general implications for the origin of Fe-Ti ores. This synthesis provides exploration perspectives and proposes realistic targets for new Fe-Ti-V-P deposits.

\section{Massif-type anorthosites and related rocks}

\subsection{Spatial and temporal distribution}

Massif-type anorthosite plutons and associated mangerite, charnockite and (rapakivi) granite (AMCG suite; Emslie, 1978; Emslie et al., 1994) occur in various terranes but are restricted to the Proterozoic
(Fig. 1; Ashwal, 1993, 2010). A distinction between andesine anorthosites $\left(\mathrm{An}_{23-48}\right)$ and labradorite anorthosites $\left(\mathrm{An}_{45-63}\right)$ was proposed by Anderson and Morin (1969). According to the experimental work of Longhi (2005) and Longhi et al. (1999), these two groups refer to different trends among the anorthosites' parental magmas that are not related by a fractional crystallization process: andesine anorthosites crystallize from magmas with higher concentrations of $\mathrm{TiO}_{2}, \mathrm{~K}_{2} \mathrm{O}$ and $\mathrm{P}_{2} \mathrm{O}_{5}$, and lower Mg\#, while magmas parental to labradorite anorthosites have lower concentrations of those three elements and usually higher $\mathrm{Mg \#}$. The most important $\mathrm{Fe}-\mathrm{Ti}$ ores are associated with andesine anorthosite (Anderson and Morin, 1969), but significant resources of Ti-magnetite also occur in labradorite anorthosites (Hébert et al., 2005; Charlier et al., 2009; Table 1).

The Mesoproterozoic Grenville Province of North America is the largest AMCG suite. Four major pulses of AMCG-type magmatism have been dated around $1320 \mathrm{Ma}, 1160-1140 \mathrm{Ma}, 1080-1050 \mathrm{Ma}$ and 1020-1008 Ma (Higgins and van Breemen, 1996; Corrigan and van Breemen, 1997; Hébert et al., 2005). The province contains many anorthosite plutons and includes the composite Havre-Saint-Pierre anorthosite, which is intruded by the Lac Tio deposit (Lister, 1966; Charlier et al., 2010b) and the Grader layered intrusion (Charlier et al., 2008). The Lac-Saint-Jean anorthosite complex has recently been explored and is dominated by titaniferous magnetite deposits in labradorite anorthosite (Hébert et al., 2005). The Labrieville anorthosite, emplaced around $1010 \mathrm{Ma}$ (Owens et al., 1994), also contains some Fe-Ti-P-rich rocks (Owens and Dymek, 1992). An overview of Fe-Ti-P-V deposits in the Grenville province has been presented by Corriveau et al. (2007).

The Rogaland Anorthosite Province, which covers ca. $1000 \mathrm{~km}^{2}$ in SW Norway (Duchesne and Korneliussen, 2003), intruded the Sveconorwegian orogenic belt of Baltica, which is correlated with the Grenvillian belt of Laurentia (Rivers et al., 1989; Romer, 1996; Rivers and Corrigan, 2000). It comprises three anorthosite plutons dated at ca. 930 Ma (Schärer et al., 1996). The Tellnes ilmenite deposit is situated in the center of the easternmost anorthosite body, the Anna-Sira anorthosite massif (Krause et al., 1985; Charlier et al., 2006).

The Damiao anorthosite was emplaced in the North China Craton at ca. 1.74 Ga (Zhang et al., 2007; Zhao et al., 2009). The area covered by anorthosite is relatively small (ca. $80-120 \mathrm{~km}^{2}$ ) but contains major ore deposits that have been mined for twenty years (Chen et al., 2013).

The AMCG suite of the Adirondack Mountains (New York), emplaced at ca. $1155 \mathrm{Ma}$ (McLelland et al., 2004), is known for the Sanford Hill

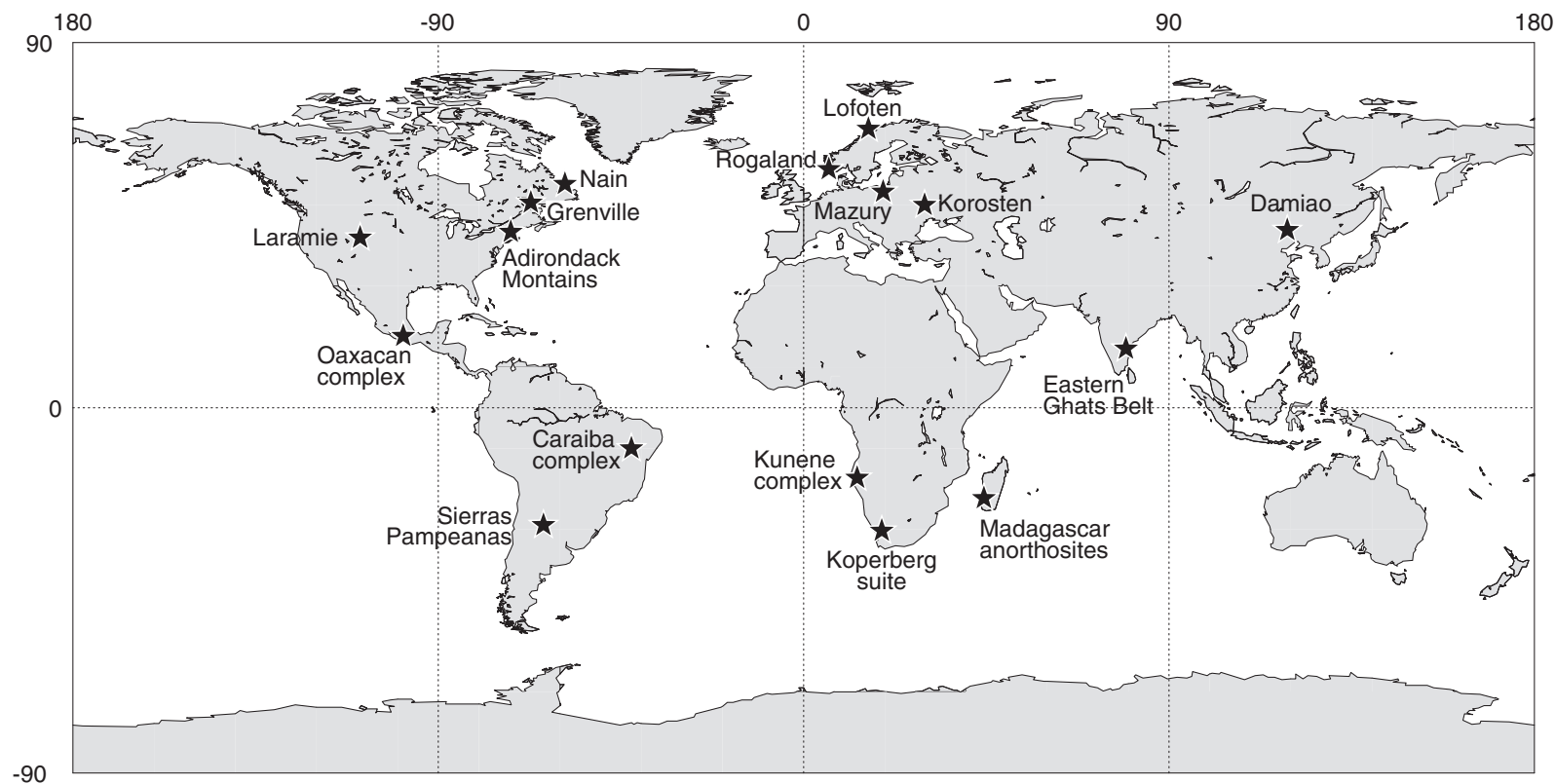

Fig. 1. Location of some anorthosite provinces with proved or potential resources of Fe-Ti and associated Cu-Ni ores (equidistant cylindrical projection). 
Table 1

Major Proterozoic anorthosite provinces containing Fe-Ti-V-P ores.

\begin{tabular}{|c|c|c|c|c|c|c|c|}
\hline $\begin{array}{l}\text { Anorthosite } \\
\text { province }\end{array}$ & Country & $\begin{array}{l}\text { Areal } \\
\text { extent }\end{array}$ & $\begin{array}{l}\text { Age } \\
(\mathrm{Ma})\end{array}$ & Type/An & Main ore deposits & Dominant ore & References \\
\hline Korosten & Ukraine & 2185 & $1789 \pm 2$ & Labradorite $/ 57.5 \pm 2.5$ & Fedorivka & Ilmenite-magnetite & $\begin{array}{l}\text { Amelin et al. (1994), } \\
\text { Duchesne et al. (2006) }\end{array}$ \\
\hline Damiao & China & 80 & $1740 \pm 20$ & Labradorite-andesine/46 \pm 5 & Damiao, Maying, Heishan & Magnetite-ilmenite & $\begin{array}{l}\text { Zhao et al. (2009), Chen } \\
\text { et al. (2013) }\end{array}$ \\
\hline Mazury & Poland & 8000 & $1559 \pm 39$ & Labradorite-andesine/50 \pm 5 & Suwalki & Magnetite-ilmenite & $\begin{array}{l}\text { Wiszniewska et al. (2002), } \\
\text { Charlier et al. (2009) }\end{array}$ \\
\hline Kunene & Angola-Namibia & 18,000 & $1385 \pm 25$ & Labradorite/66.5 \pm 9.5 & Oryeheke $+\mathrm{Ni}-\mathrm{Cu}$ deposits & Ilmenite & Maier et al. (2013) \\
\hline Laramie & Wyoming, USA & 800 & $1434 \pm 3$ & Labradorite-andesine/50 \pm 10 & $\begin{array}{l}\text { Iron Mountain, Sybille, } \\
\text { Strong Creek }\end{array}$ & Ilmenite-magnetite & $\begin{array}{l}\text { Scoates and Chamberlain } \\
\text { (1997), Lindsley (2003) }\end{array}$ \\
\hline $\begin{array}{l}\text { Rivière } \\
\text { Pentecôte }\end{array}$ & Quebec, Canada & 600 & $1354 \pm 3$ & Labradorite-andesine/50 \pm 5 & Rivière Pentecôte & Ilmenite-magnetite & $\begin{array}{l}\text { Martignole et al. (1993), } \\
\text { Francis et al. (2000) }\end{array}$ \\
\hline De La Blache & Quebec, Canada & 1500 & $1327 \pm 16$ & Labradorite/55 \pm 2 & $\begin{array}{l}\text { Hervieux, Schmoo, Lac } \\
\text { Dissimieu }\end{array}$ & Ilmenite-magnetite & Hébert et al. (2005) \\
\hline Lac St Jean & Quebec, Canada & 20,000 & $1156 \pm 2$ & Labradorite/60.5 \pm 1.5 & $\begin{array}{l}\text { Lac a Paul, Saint Charles, } \\
\text { Buttercup }\end{array}$ & Magnetite-ilmenite & $\begin{array}{l}\text { Higgins and van Breemen } \\
\text { (1996), Hébert et al. (2005) }\end{array}$ \\
\hline $\begin{array}{l}\text { Valin } \\
\text { (Labrieville; } \\
\text { Mattawa) }\end{array}$ & Quebec, Canada & 1265 & $1010 \pm 5$ & Andesine $/ 35 \pm 5$ & $\begin{array}{l}\text { Mirepoix, la Hache, Lac } \\
\text { Brule }\end{array}$ & Magnetite-ilmenite & $\begin{array}{l}\text { Hébert et al. (2005), } \\
\text { Morisset et al. (2013) }\end{array}$ \\
\hline Adirondacks & New York, USA & 3000 & $1154 \pm 6$ & Labradorite-andesine/49 \pm 6 & Sanford Lake & Magnetite-ilmenite & $\begin{array}{l}\text { McLelland et al. (2004), } \\
\text { Morisset et al. (2013) }\end{array}$ \\
\hline Morin & Quebec, Canada & 2500 & $1155 \pm 3$ & Labradorite-andesine/48 \pm 6 & $\begin{array}{l}\text { Degrosbois, Ivry, } \\
\text { Saint-Hippolyte }\end{array}$ & Ilmenite-magnetite & $\begin{array}{l}\text { Doig (1991), Morisset et al. } \\
\text { (2013) }\end{array}$ \\
\hline Oaxaca & Mexico & 400 & $1012 \pm 12$ & Andesine $/ 46 \pm 2$ & Pluma Hidalgo & Rutile & $\begin{array}{l}\text { Force (1991), Keppie et al. } \\
\text { (2003) }\end{array}$ \\
\hline Havre St Pierre & Quebec, Canada & 5500 & $1060 \pm 3$ & Andesine $/ 42.2 \pm 2.5$ & $\begin{array}{l}\text { Grader, Allard Lake, Big } \\
\text { Island, Everett }\end{array}$ & Ilmenite & Morisset et al. (2009) \\
\hline Saint-Urbain & Quebec, Canada & 450 & $1053 \pm 3$ & Labradorite-andesine/50 \pm 15 & $\begin{array}{l}\text { Coulombe, Bignel, Dupont, } \\
\text { General Electric }\end{array}$ & Ilmenite & Morisset et al. (2009) \\
\hline Rogaland & Norway & 580 & $931 \pm 2$ & Labradorite-andesine/47.5 \pm 7.5 & $\begin{array}{l}\text { Tellnes, Storgangen, Hesnes, } \\
\text { Bjerkreim-Sokndal }\end{array}$ & Ilmenite & Schärer et al. (1996) \\
\hline $\begin{array}{l}\text { Malagasy } \\
\text { graphite } \\
\text { sequence }\end{array}$ & Madagascar & 250 & $660 \pm 60$ & Labradorite-andesine/47.5 \pm 6.5 & & & Ashwal et al. (1998) \\
\hline
\end{tabular}

deposit (Gross, 1968). The anorthosite complex also contains oxideapatite gabbronorites (Ashwal, 1982; McLelland et al., 1994; Seifert et al., 2010). The Laramie anorthosite complex (SE Wyoming), dated at $1.43 \mathrm{Ga}$ (Scoates and Chamberlain, 2003), is closely associated with the Horse Creek anorthosite complex (1.78 Ga; Scoates and Chamberlain, 1997). Several Fe-Ti ores are present here, of which Iron Mountain, the Sybille pit and Strong Creek have been described (Lindsley et al., 1988; Frost and Simons, 1991).

The East European Craton contains two major AMCG suites: the Mazury complex and the Korosten Pluton. The Mazury complex, situated in northeastern Poland, is covered by a thick sequence of sedimentary rocks and is known only from drill-cores studies. It is a $200 \mathrm{~km} \mathrm{E-W}$ trending complex with two large anorthosite plutons (Wiszniewska et al., 2002; Skridlaite et al., 2003; Duchesne et al., 2010), including the Suwalki anorthosite. Fe-Ti deposits in this anorthosite, dominated by Ti-magnetite (Charlier et al., 2009), are dated at ca. $1560 \mathrm{Ma}$ (Re-Os ages; Morgan et al., 2000). The Korosten Pluton occupies about $12,500 \mathrm{~km}^{2}$ of the northwestern part of the Ukrainian shield. It contains three massif-type anorthosites and associated rocks, which were emplaced between 1800 and $1740 \mathrm{Ma}$ (see the review of Bogdanova et al., 2004). In this province, the Fedorivka layered intrusion has significant potential for Fe-Ti ores (Duchesne et al., 2006).

The Kunene Complex of SW Angola and NW Namibia covers 15,000$18,000 \mathrm{~km}^{2}$ and is probably made up of several individual plutons with small Fe-Ti ore bodies (Ashwal and Twist, 1994; Gleissner et al., 2010, 2011; Maier et al., 2013). U-Pb dating of zircon in a mangerite from this complex gives an age of $1371 \pm 2.5 \mathrm{Ma}$ (Mayer et al., 2004).

Many other anorthosite complexes have been described (see the review of Ashwal, 2010), in which no significant Fe-Ti resources have been studied or discovered. The Lofoten-Vesterålen AMCG suite in northern Norway (Markl et al., 1998; Markl and Frost, 1999) was emplaced ca. 1.8 Ga (Corfu, 2004) into Early Proterozoic supracrustals and Archean gneisses. Massive and disseminated Fe-Ti concentrations occur in gabbros and anorthosites, and the layered Selvåg gabbroic intrusion (Priesemann and Krause, 1985) represents a minor potential for Fe-Ti ore. Massif-type anorthosites also outcrop in southwest Madagascar (Ashwal et al., 1998) and India, which were both part of East Gondwana. The Eastern Ghats Belt of India hosts four massif-type anorthosite complexes (Bhattacharya et al., 1998; Krause et al., 2001; Dobmeier and Simmat, 2002; Dobmeier, 2006; Chatterjee et al., 2008). Other massifs are also described in south India (Janardhan and Wiebe, 1985), but no ore bodies have been discovered. Anorthosites from the northern Oaxacan Complex (S Mexico), dated at ca. 1012 Ma (Keppie et al., 2003), are correlated with Grenvillian AMCG suite. Casquet et al. (2005) have reported the discovery of massif-type anorthosites covering ca. $50 \mathrm{~km}^{2}$ in the Andean basement of the Western Sierras Pampeanas of Argentina, including an occurrence of magnetite-apatite-rich rock (nelsonite). U-Pb zircon dating of a gabbronorite dyke yields an age for these anorthosites of ca. 1070 Ma. The Nain Plutonic Suite of Labrador, Canada (Emslie, 1978; Emslie et al., 1994; Ryan, 2000; Myers et al., 2008) has no Fe-Ti deposits but hosts the giant Ni-Cu-Co deposit of the Voisey's Bay intrusion (Li et al., 2000). Two important cupriferous deposits related to anorthosites are found in the Koperberg Suite in South Africa (Conradie and Schoch, 1986; Gibson et al., 1996; Clifford et al., 2004; Duchesne et al., 2007) and the Caraiba complex in Brazil (Oliveira and Tarney, 1995; Maier and Barnes, 1996, 1999). Magnetite-hypersthene ores have been described in these localities but Ti-resources are absent.

\subsection{Crystallization conditions of anorthosites}

Massif-type anorthosites are thought to crystallize in two stages (Duchesne et al., 1985a, 1999; Emslie, 1985; Ashwal, 1993). The first involves the accumulation of plagioclase at the top of deep-seated 
magma chambers (Kushiro and Fujii, 1977; Kushiro, 1980), followed by the intrusion of buoyant plagioclase mushes into mid-crustal levels. The ascent of the plagioclase crystal mush from the upper part of the deep magma chamber results from gravitational instability due to its low density relative to the crust. Even though the emplacement of an anorthositic massif by simple vertical diapirism has been successfully modeled (Barnichon et al., 1999), the intrusions are probably favored by zones of crustal weakness such as major lineaments (Corrigan and Hanmer, 1997; Scoates and Chamberlain, 1997; Duchesne et al., 1999; Ryan, 2000; Bogdanova et al., 2004).

The polybaric crystallization of anorthosites is largely deduced from the occurrence of high-alumina orthopyroxene megacrysts (HAOM) containing plagioclase exsolutions (Emslie, 1975). Experiments on the stability of HAOM reveal that the Al-richest compositions are stable at pressures of 11-13 kbar (Fram and Longhi, 1992; Longhi et al., 1993, 1999). The alumina content of orthopyroxene commonly displays a continuous range from 2 to 8 wt.\% $\mathrm{Al}_{2} \mathrm{O}_{3}$, supporting continuous polybaric crystallization during diapiric rise of the anorthositic mush (Charlier et al., 2010a). Final pressures of emplacement estimated from mineral assemblages in the contact metamorphic aureoles range from 3 to 6 kbar (Berg, 1977; Jansen et al., 1985; Westphal et al., 2003).

Deformation features in anorthosites, such as strongly foliated margins and the ubiquitous dynamic recrystallization of plagioclase (primary crystals are replaced by smaller recrystallized grains; e.g. Lafrance et al., 1996; Nasipuri and Bhattacharya, 2007), are syn-emplacement features and can be accounted for by anorthosite diapirism and ballooning (Martignole and Schrijver, 1970; Barnichon et al., 1999). Gravitational instabilities are also responsible for the sagging of dense Fe-rich rocks in host anorthosite (Paludan et al., 1994; Bolle et al., 2000, 2002).

Anorthosite plutons in the Laramie complex do not have diapiric structures; they appear to be magma chambers with characteristics typical of mafic layered intrusions. They display a well-defined stratigraphy with alternating anorthosite and leucocratic gabbroic rocks (Lindsley et al., 2010; Scoates et al., 2010). The building of these plutons is interpreted as resulting from successive injections of magma containing large amounts of plagioclase megacrysts. This emplacement scenario is similar to that of the anorthositic base of the Bjerkreim-Sokndal layered intrusion in the Rogaland Anorthosite Province (Wilson et al., 1996; Duchesne and Charlier, 2005).

Various tectonic settings have been proposed for massif-type anorthosites, from anorogenic intraplate to convergent and divergent settings (McLelland et al., 2010). However, most anorthosite provinces seem to be associated with major lineaments (Corrigan and Hanmer, 1997; Scoates and Chamberlain, 1997; Duchesne et al., 1999; Ryan, 2000; Bogdanova et al., 2004), and a consensus is emerging that anorthosites were emplaced in continental arc environments in a post-collisional regime (Duchesne et al., 1999; Zhang et al., 2007; Vander Auwera et al., 2011; Bybee et al., 2014).

Anorthosite complexes are emplaced into high-grade metamorphic rocks and are surrounded by an aureole of contact metamorphism (Berg, 1977) up to $20 \mathrm{~km}$ thick (Westphal et al., 2003; Drüppel et al., 2013). Granulite-facies assemblages with orthopyroxene, pigeonite or osumilite are common (Möller et al., 2003).

\subsection{Composition and source for parental magmas: mantle vs. lower crust}

The compositions of proposed magmas parental to massif-type anorthosite range from high-Al basalt to jotunite (hypersthene-bearing monzodiorite; Emslie, 1980; Fram and Longhi, 1992; Mitchell et al., 1995; Vander Auwera et al., 1998; Charlier et al., 2010a). The major contention concerning the petrogenesis of anorthosite is whether parental magmas are produced by melting the mantle (Emslie, 1978; Ashwal, 1993; Bybee et al., 2014) or by melting a mafic lower crust (Duchesne et al., 1999; Longhi et al., 1999; Longhi, 2005). Rb-Sr and
Sm-Nd isotopic studies (e.g. Taylor et al., 1984; Emslie et al., 1994; Scoates and Frost, 1996) have not been able to unambiguously determine the origin of these parental magmas because they cannot readily distinguish a mantle-derived magma with crustal contamination from a melt of mafic lower crust with only brief crustal residence (Demaiffe et al., 1986). Re-Os isotopic studies on sulfide minerals in the Suwalki anorthosite (Morgan et al., 2000) and the Rogaland Anorthosite Province (Schiellerup et al., 2000) yield high initial ${ }^{187} \mathrm{Os} /{ }^{188}$ Os ratios, which require a crustal source. However, these interpretations have been questioned by Hannah and Stein (2002) who have modeled similar high initial ${ }^{187} \mathrm{Os} /{ }^{188} \mathrm{Os}$ by direct assimilation of crustal sulfide by a mantle-derived magma. Further determination of initial ${ }^{187} \mathrm{Os} /{ }^{188} \mathrm{Os}$ on silicates and oxides would be useful.

Experimental constraints on liquidus equilibria under dry conditions (Longhi et al., 1999) have revealed a thermal divide on the plagioclase + pyroxene liquidus surface at 10-12 kbar. This suggests that a magma parental to anorthosite cannot be a mantle melt contaminated by the crust but should instead result from the direct melting of a gabbronoritic source. Moreover, calculations of fractional crystallization with and without assimilation (Longhi, 2005) have shown that it is not possible to produce a high-Al liquid by melting typical mantle peridotites or even anomalous $\mathrm{Fe}$ - and Al-enriched mantle (Olsen and Morse, 1990) with sufficient $\mathrm{TiO}_{2}$ to make andesine-type anorthosite. The alternative proposed by Longhi et al. (1999) involves partial melting of a mafic lower crust, which is more suitable for producing $\mathrm{TiO}_{2}$-rich magmas.

\subsection{Related rocks: comagmatic or simply coeval with massif-type anorthosites?}

Massif-type anorthosites are commonly associated with rocks of intermediate composition. These are referred to as ferrodiorite (Emslie, 1985; Mitchell et al., 1996), monzonorite (Duchesne et al., 1974, 1985b) and jotunite (Owens et al., 1993; Vander Auwera et al., 1998), and are all significantly enriched in Fe-Ti-P. They occur as fine-grained rocks in dykes, chilled margins of leuconorite plutons (e.g. Hidra body; Demaiffe and Hertogen, 1981) and layered intrusions (e.g. Bjerkreim-Sokndal; Duchesne and Hertogen, 1988), as well as cumulates in (layered) intrusions (Duchesne et al., 1987). Anorthosites can be associated with more felsic compositions: mangerite (hypersthene monzonite), quartz mangerite (hypersthene quartz monzonite) and charnockite (hypersthene granite) (Duchesne and Wilmart, 1997).

Two major hypotheses are invoked for the origin of rocks associated with massif-type anorthosites: (1) they are residual liquids after the crystallization of anorthosite (Emslie, 1978; Morse, 1982; Emslie et al., 1994; Mitchell et al., 1996; Scoates et al., 1996); (2) they are the direct product of partial melting of a lower gabbronoritic crust and are the parental magmas of andesine anorthosites (Duchesne and Demaiffe, 1978; Longhi et al., 1999). These two hypotheses are not mutually exclusive; most authors agree that these rocks represent intermediate compositions in the comagmatic sequence from anorthosite to mangerite and even to granitoids. Experimental studies (Vander Auwera et al., 1998) have shown that evolved monzonitic and mangeritic rocks can be produced by closed-system fractional crystallization from jotuniticferrodioritic magmas, although variable degrees of crustal contamination might also be involved (Bolle et al., 2003). Scoates and Lindsley (2000) suggest an important role for polybaric fractional crystallization in producing compositional diversity in Proterozoic anorthosite plutonic suites. The comagmatic origin of jotunitic to charnockitic melts is also evidenced by the Tellnes dyke, closely associated with the Tellnes ilmenite deposit, which is made up of this continuous series of compositions and can be modeled by closed-system fractional crystallization (Wilmart et al., 1989).

Massif-type anorthosites are also commonly associated with ferroan A-type granite and charnockite (Emslie, 1978; Duchesne and Wilmart, 
1997; Frost and Frost, 2011, 2013). None of these silicic rocks contain any Fe-Ti-V-P ores.

\section{Characteristics of major deposits}

Three rock deposits are currently mined: Lac Tio (Quebec), Tellnes (SW Norway), and Damiao (China). Together, these mines produce 5-6 million tons of ore with contrasting grades and ilmenite compositions (Fig. 2). Each deposit also has its own structure, internal variations and relationships with the host anorthosite as detailed below.

\subsection{Lac Tio, Quebec}

The Lac Tio (or Allard Lake) hemo-ilmenite ore body is situated on the north shore of the Saint Lawrence estuary (Quebec). The deposit outcrops in the $1.06 \mathrm{Ga}$ Lac Allard anorthosite, which is part of the Havre-Saint-Pierre anorthosite suite of the Grenville province in North America (Emslie, 1978; Perreault and Hébert, 2003; Morisset et al., 2009). It was discovered in June 1946 in the Allard Lake area near a small lake named Lac Tio, during the first aeromagnetic survey for ore exploration (Bourret, 1949). Following two years of drilling by Kennco Explorations, it has been continuously exploited since 1951 as an open-pit mine. The ore body is the world's largest known hard-rock ilmenite deposit, with current reserves estimated at ca. $138 \mathrm{Mt}$ at grades exceeding $60 \mathrm{wt}$.\% hemo-ilmenite. The $\mathrm{TiO}_{2}$ content of the ore is variable but is mainly between 32 and $38 \mathrm{wt} . \% \mathrm{TiO}_{2}$, which is much higher than the Tellnes and Damiao deposits (Fig. 2).

The main ore body is a funnel-shaped intrusion measuring $1.03 \times$ $1.10 \mathrm{~km}$ and 100-300 m thick. Two smaller bodies are separated from the main deposit by faults and anorthosite. The ore is an ilmenite-rich norite (or ilmenitite) made up of hemo-ilmenite ( $\mathrm{Hem}_{22.6-29.4}, 66.2 \mathrm{wt} . \%$ on average), andesine plagioclase $\left(\mathrm{An}_{45-50}\right)$, aluminous spinel and locally orthopyroxene. Compared to other deposits, ilmenite in Lac Tio is significantly richer in hematite and has a moderate $\mathrm{MgO}$ content (2.5-3.0 wt.\%; Fig. 3).

The ilmenite-rich body has usually been considered to have formed as an enormous drop of immiscible Fe-Ti-enriched liquid separated from the magma remaining after the crystallization of the andesine anorthosite (Hammond, 1952; Lister, 1966). However, whole-rock compositions are controlled by the proportions of ilmenite and plagioclase \pm orthopyroxene, which supports a cumulate origin for the deposit (Charlier et al., 2010b). Cr concentrations in ilmenite reveal normal and reverse fractionation trends, suggesting multiple episodes of magma emplacement and alternating periods of fractional crystallization and magma mixing. This mixing produced hybrid magmas located in the stability field of ilmenite, resulting in periodic crystallization of ilmenite alone. The unsystematic differentiation trends in the Lac
Tio deposit, arising from a succession of magma pulses, hybridization, and the fractionation of hemo-ilmenite alone or together with plagioclase, suggest that the deposit formed within a magma conduit (Charlier et al., 2010b). This dynamic emplacement mechanism associated with continuous gravity-driven accumulation of Fe-Ti oxides, and possibly plagioclase buoyancy in a fractionating ferrobasalt, explains the huge concentration of hemo-ilmenite.

\subsection{Tellnes, SW Norway}

The Tellnes ore body is an ilmenite-rich norite averaging slightly more than $18 \% \mathrm{TiO}_{2}$. It was discovered in 1954 during an aeromagnetic survey, and has been mined since 1960 by TITANIA A/S as an open-pit (Krause et al., 1985). Reserves are estimated at $57 \mathrm{Mt} \mathrm{TiO}_{2}$, representing $14 \%$ of the world reserves of ilmenite and $12 \%$ of the total world reserves of titanium minerals (ilmenite + rutile). By-products are magnetite and $\mathrm{Ni}-\mathrm{Cu}$ sulfide concentrates. The Tellnes ore body is intruded into the Åna-Sira anorthosite, which is part of the Rogaland anorthosite province in SW Norway. The deposit crosscuts the anorthosite and thus is younger than its host; zircon U-Pb ages are $920 \pm 3$ Ma for the ore body and $932 \pm 3$ Ma for the anorthosite (Schärer et al., 1996). It has a sickleshaped outcrop oriented WNW-ESE to NNW-SSE with a maximum width and length of $400 \mathrm{~m}$ and $2700 \mathrm{~m}$, respectively. Its 3D shape is that of a gently plunging elongated trough. The intrusive character of the ore body is evident from sharp contacts with the host anorthosite, numerous apophyses and anorthosite xenoliths. The $\mathrm{TiO}_{2}$ content of the ore is usually 16-20 wt.\% (Fig. 2b), with concentrations generally the highest in the center of the ore body and decreasing towards the margins (Charlier et al., 2006). The MgO content of ilmenite (1.44.4 wt.\%; Fig. 3b) is systematically lower in samples from the margins of the ore body. This has been interpreted to be due to extensive postcumulus re-equilibration with trapped liquid and ferromagnesian silicates, and is correlated with distance to the host anorthosite (Charlier et al., 2007). The hematite content varies slightly between $\mathrm{Hem}_{10}$ and $\mathrm{Hem}_{14}$ and is systematically higher in samples from the margins of the ore body.

Tellnes was previously described as a rather homogeneous ore body with plagioclase, ilmenite, and orthopyroxene as the major minerals (Gierth and Krause, 1973). However, the deposit displays significant variation in the compositions and proportions of minerals (Charlier et al., 2006). The lower part of the ore body mainly contains plagioclase and ilmenite. Upsection, orthopyroxene and olivine appear. Marginal zones are characterized by high plagioclase and apatite contents and lower proportions of ilmenite. Variations in ilmenite composition with stratigraphic height indicate that fractional crystallization was the major differentiation process in the Tellnes ilmenite deposit. The calculated cotectic proportion of ilmenite in plagioclase-ilmenite cumulates
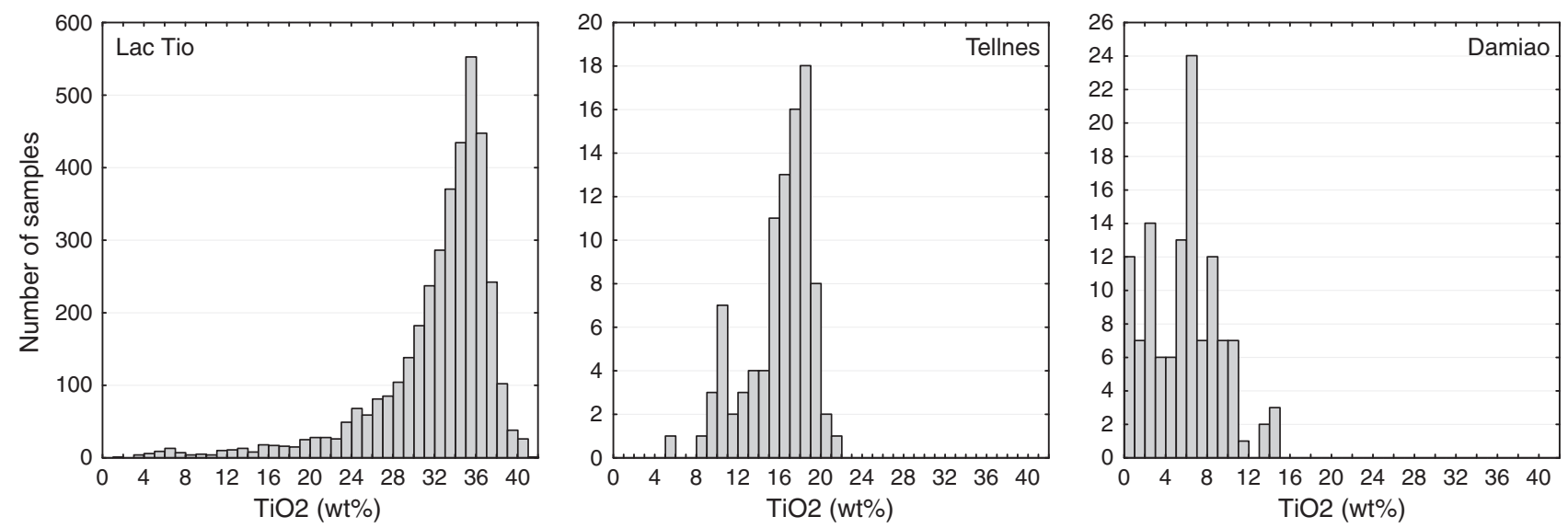

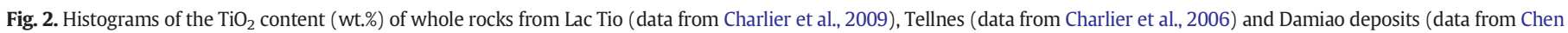
et al., 2013). 

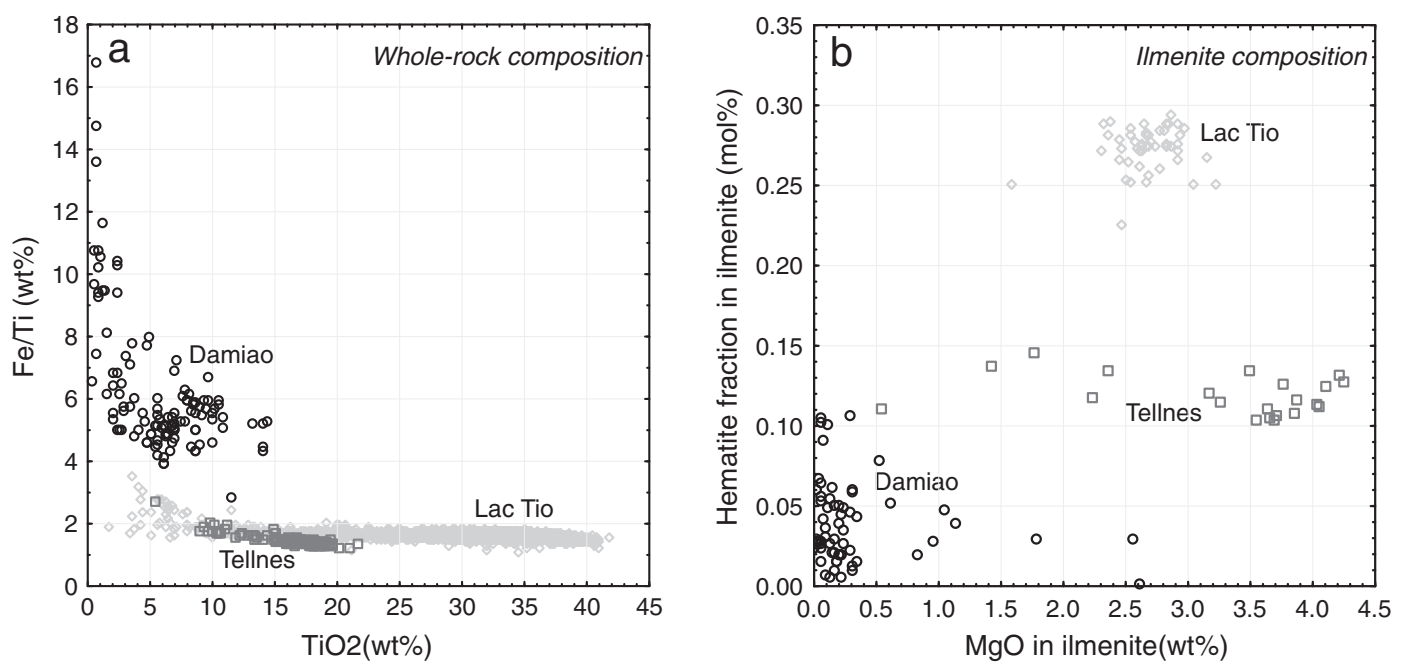

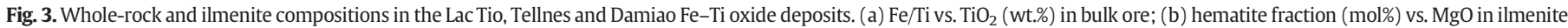
(data from Charlier et al., 2006, 2007, 2009; Chen et al., 2013).

is $17.5 \%$, significantly lower than the proportion of cumulus ilmenite in the bulk deposit (ca. 50\%; Charlier et al., 2007). This implies sorting of ilmenite and its preferential accumulation at the bottom of the chamber, which has been interpreted to have had a sill shape that is now deformed into an elongated trough (Charlier et al., 2006).

\subsection{Damiao, China}

The Damiao deposit is hosted in the ca. 1.74 Ga Damiao andesine anorthosite complex, located in the northern part of the North China Craton (Xie, 1982; Zhao et al., 2009). The magmatic province hosts a complete suite of rocks related to anorthosite, i.e. norite, gabbronorite, ferrodiorite, mangerite, alkali granitoid, and rapakivi granite (Zhang et al., 2007; Zhao et al., 2009; Chen et al., 2013). Trace element and isotopic evidence ( $\mathrm{Sr}-\mathrm{Nd}-\mathrm{Hf}$ ) support derivation of the magmatic suite by fractional crystallization of one parental high-alumina basalt (Zhang et al., 2007; Zhao et al., 2009). The Damiao deposit has been mined in open pits and underground for several decades at an annual production of $2 \mathrm{Mt}$ ore with economic concentrations of Fe, Ti, P and $\mathrm{V}$. The deposit is subdivided into many discordant ore bodies (more than 90) occurring as irregular lenses, veins or pods crosscutting the anorthosite (Sun et al., 2009; Chen et al., 2013; Li et al., 2014). The ore has an average grade of about $36 \mathrm{wt} . \% \mathrm{Fe}_{2} \mathrm{O}_{3 \text { tot }}, 7.0 \mathrm{wt}$.\% $\mathrm{TiO}_{2}, 0.3 \mathrm{wt} . \%$ $\mathrm{V}_{2} \mathrm{O}_{5}$, and 2.0 wt.\% $\mathrm{P}_{2} \mathrm{O}_{5}$ (Sun et al., 2009). The ore is hosted by massive anorthosite and locally by leuconorite. Various types of ore rocks are identified (Li et al., 2014): massive Fe ore with Ti-magnetite and ilmenite (Fe-Ti ore); massive P ore containing more than $50 \%$ apatite (nelsonitic ore); massive Fe-P ore with apatite, Ti-magnetite and ilmenite (oxide-apatite gabbronorite ore); and disseminated $\mathrm{Fe}$ and Fe-P ore representing more than $60 \%$ of the total reserve. Unlike Tellnes and Lac Tio, the ore is dominated by Ti-magnetite, which explains the high $\mathrm{Fe} / \mathrm{Ti}$ ratio (Fig. 3a). The whole-rock $\mathrm{TiO}_{2}$ content of the Damiao deposit is also comparatively low, mainly between 6 and 12 wt.\% (Fig. 2c). However, the composition of the ilmenite is of much higher quality with low $\mathrm{Cr}$ and $\mathrm{Mg}$ (Chen et al., 2013). Ilmenite contains less than 0.5 wt.\% $\mathrm{MgO}$, and has a low hematite fraction $(<7 \mathrm{~mol} \%$; Fig. 3b). Immiscibility, promoted by oxidizing crystallization conditions and high phosphorous concentration in the magma, and formation of a nelsonitic melt (Fe-Ti-P-rich ferrobasalt) has been proposed as a major ore-forming process (Chen et al., 2013). The crystallization products of this melt, enriched in Ti-magnetite, ilmenite \pm apatite, would have been accumulated in residual melt pockets of the host anorthosite. Hydrothermal remobilization of $\mathrm{Fe}$ and $\mathrm{Ti}$ from the host altered anorthosite has also been suggested for the formation of massive Fe-P ores (Li et al., 2014).

\section{Mineralization styles, grades and sizes}

The three major deposits described above have different mineralization styles. Internal variations in Fe-Ti deposits and relationships with the host anorthosite and associated rocks are even more complex when considering the huge number of minor deposits mentioned in the literature. Based on the major characteristics of selected occurrences of Fe-Ti $\pm \mathrm{P}$ (Table 2 ), a few major mineralization styles can be defined. Rose (1969) asserted that these deposits could occur within tabular intrusives, stocks, sills or dykes in massive anorthosites but also "locally as stratified accumulations in layered segments of anorthosite massifs or in layered intrusions". Compared to major deposits, other occurrences have significantly lower economic potential, as shown by lower titanium grades and tonnage (Fig. 4), although some of them have been exploited in the past, and still contain significant resources.

\subsection{Massive deposits}

Hemo-ilmenite ( \pm Ti-magnetite in some deposits) occurs in massive deposits and commonly constitutes more than $90 \%$ of the mineral assemblage. Accompanying phases are typically plagioclase, orthopyroxene, and spinel. The rock is usually coarse-grained, with polygonal, tabular crystals of hemo-ilmenite. Massive deposits are usually discordant with the host anorthosite and can be variably deformed. The Lac Tio deposit is the main example of massive ore, even though more plagioclase-rich parts of the deposit display a conspicuous modal layering. Other examples of massive ore are Jerneld, Blåfjell and part of Kydlandsvatn in the Rogaland province (ilmenitite); part of the Iron Mountain deposit in Wyoming (ilmenite + magnetite); and St Urbain deposits in the Grenville province (ilmenite + rutile).

\subsection{Stratiform deposits}

Stratiform deposits are found in intrusions that are clearly distinct from massif-type anorthosites. Major examples occur in the Layered Series of the Bjerkreim-Sokndal layered intrusion, which outcrops over $230 \mathrm{~km}^{2}$ and consists of a 7000 m-thick sequence of cumulates made up of anorthosite, troctolite, leuconorite, norite, gabbronorite, jotunite and mangerite (Wilson et al., 1996). Fe-Ti-P-enriched rocks occur at the top of the Layered Series, where huge volumes of rocks with 5-7 wt.\% $\mathrm{TiO}_{2}$ and 4-5 wt.\% $\mathrm{P}_{2} \mathrm{O}_{5}$ have been reported (Duchesne 
Table 2

Principal characteristics of representative Fe-Ti oxide deposits.

\begin{tabular}{|c|c|c|c|c|c|c|c|}
\hline Deposit name & Location & Setting & Rock type & Main paragenesis & Oxide composition & Suggested origin & References \\
\hline Jerneld & $\begin{array}{l}\text { Helleren anorthosite, } \\
\text { Rogaland, SW Norway }\end{array}$ & $\begin{array}{l}\text { Veins in massive } \\
\text { anorthosite }\end{array}$ & $\begin{array}{l}\text { Massive } \\
\text { hemo-ilmenite }\end{array}$ & Ilmenite \pm Al-spinel \pm sulfides \pm plag & $\begin{array}{l}\text { Cr-, V-, Mg-rich hemo-ilmenite } \\
\text { (Hem } 18 ; \mathrm{MgO}: 5.3 \% ; \mathrm{Cr}: 0.3 \% ; \mathrm{V} \text { : } \\
0.22 \%)\end{array}$ & $\begin{array}{l}\text { Remobilized ilmenite } \\
\text { cumulate }\end{array}$ & 1 \\
\hline Blåfjell & $\begin{array}{l}\text { Åna-Sira anorthosite, } \\
\text { Rogaland, SW Norway }\end{array}$ & Bodies in noritic pegmatite & Massive ilmenitite & Ilmenite \pm plag $\left(\mathrm{An}_{40-50}\right)$ & $\begin{array}{l}\mathrm{Cr}-, \mathrm{V}-\mathrm{Mg} \text {-rich hemo-ilmenite } \\
\left(\mathrm{Hem}_{20} ; \mathrm{MgO}: 5.7 \% ; \mathrm{Cr}: 0.2 \% ; \mathrm{V}:\right. \\
0.20 \%)\end{array}$ & Cumulate in noritic melt & 1,2 \\
\hline Tellnes & $\begin{array}{l}\text { Åna-Sira anorthosite, } \\
\text { Rogaland, SW Norway }\end{array}$ & $\begin{array}{l}\text { Dyke-like intrusion in } \\
\text { massive anorthosite }\end{array}$ & $\begin{array}{l}\text { Homogeneous } \\
\text { ilmenite norite, } \\
\text { locally laminated }\end{array}$ & $\begin{array}{l}\text { Plag }\left(\mathrm{An}_{42-45}\right)+\mathrm{opx}\left(\mathrm{En}_{77-75}\right) \pm \mathrm{ol}\left(\mathrm{Fo}_{80}\right)+\mathrm{Fe}-\mathrm{Ti} \\
\text { oxides }\end{array}$ & $\begin{array}{l}\text { Hemo-ilmenite }\left(\mathrm{Hem}_{10-15} \text {; MgO: }\right. \\
\text { 1.4-4.2\%; Cr: 0.01-0.21\%; V: } \\
0.10-0.16 \%) \pm \text { magnetite (Cr: } 0.8 \% \\
\text { V: } 0.51 \%)\end{array}$ & $\begin{array}{l}\text { Cumulate enriched by } \\
\text { crystal sorting in a } \\
\text { ferrodioritic liquid }\end{array}$ & 3,4 \\
\hline Storgangen & $\begin{array}{l}\text { Åna-Sira anorthosite, } \\
\text { Rogaland, SW Norway }\end{array}$ & $\begin{array}{l}\text { Concordantly layered dyke } \\
\text { intruded in massive } \\
\text { anorthosite }\end{array}$ & $\begin{array}{l}\text { Melanoritic layers } \\
\text { in slightly deformed } \\
\text { layered norite }\end{array}$ & Plag $\left(\mathrm{An}_{43-55}\right)+\mathrm{opx}\left(\mathrm{En}_{70-75}\right)+\mathrm{Fe}-\mathrm{Ti}$ oxides & $\begin{array}{l}\text { Hemo-ilmenite }\left(\mathrm{Hem}_{13} \text {; MgO: } 3.3 \%\right. \\
\text { Cr: } 0.03 \% \text {; V: } 0.2 \%)+ \text { magnetite (Cr: } \\
0.2-0.7 \% \text {; V: } 0.65 \%)\end{array}$ & $\begin{array}{l}\text { Cumulate in a } \\
\text { differentiated sill }\end{array}$ & $1,5,6,6 \mathrm{~b}$ \\
\hline Kydlandsvatn & Rogaland, SW Norway & $\begin{array}{l}\text { Strongly dipping ilmenite } \\
\text { layers in the contact zone } \\
\text { between Egersund-Ogna } \\
\text { and Helleren anorthosites }\end{array}$ & $\begin{array}{l}\text { Ilmenite layers in } \\
\text { layered anorthosite } \\
\text { and leuconorite, } \\
\text { local nelsonite }\end{array}$ & Ilmenite \pm plag $\left(\mathrm{An}_{40-50}\right) \pm \mathrm{opx} \pm$ apatite & $\begin{array}{l}\text { Hemo-ilmenite }\left(\mathrm{Hem}_{15-25} \text { MgO: }\right. \\
3.5 \% \text {; } \mathrm{Cr}:<0.03 \% ; \mathrm{V} \text { : } \\
0.25 \%) \pm \text { spinel } \pm \text { magnetite }(\mathrm{Cr}: \\
0.2 \% \text {;: } 0.7 \%)\end{array}$ & $\begin{array}{l}\text { Cumulate in a } \\
\text { differentiated sill } \\
\text { plastically deformed by the } \\
\text { anorthosite emplacement } \\
\text { process }\end{array}$ & 1 \\
\hline Bjerkreim-Sokndal & Rogaland, SW Norway & $\begin{array}{l}\text { Gabbronorite layers in a } \\
\text { layered intrusion }\end{array}$ & $\begin{array}{l}\text { Oxide apatite } \\
\text { gabbronorite }\end{array}$ & $\begin{array}{l}\text { Plag }\left(\mathrm{An}_{37-42}\right)+\text { opx }\left(\mathrm{En}_{55-67}\right)+\mathrm{Fe}-\mathrm{Ti} \\
\text { oxides }+\mathrm{cpx}+\text { apatite }\end{array}$ & $\begin{array}{l}\text { Cr-, Mg-poor ilmenite }(\mathrm{Hem}<7 \text {; } \\
\mathrm{MgO}<1 \% ; \mathrm{Cr}<0.007 \%)+\mathrm{V} \text {-rich } \\
\text { magnetite }(\mathrm{V}: 0.3-0.7 \%)\end{array}$ & $\begin{array}{l}\text { Two-poles cumulates in a } \\
\text { ferrodioritic melt }\end{array}$ & 7,8 \\
\hline Hesnes & Rogaland, SW Norway & $\begin{array}{l}\text { Veins in the contact zone } \\
\text { between Egersund-Ogna } \\
\text { and Håland anorthosites }\end{array}$ & $\begin{array}{l}\text { Nelsonite with } \\
\text { planar orientation }\end{array}$ & Fe-Ti oxides + apatite + sulfides \pm zircon & $\begin{array}{l}\text { Ti-rich magnetite }\left(\mathrm{Usp}_{22-31} \text {; } \mathrm{Cr} \text { : }\right. \\
0.01-0.18 \% ; \mathrm{V}: 0.21 \%)+\mathrm{Cr}-, \mathrm{V} \text {-poor } \\
\text { ilmenite }\left(\mathrm{Hem}_{5} ; \mathrm{Cr}<0.01 \% \text {; } \mathrm{V} \text { : }\right. \\
0.02 \%)\end{array}$ & $\begin{array}{l}\text { Cumulate in an immiscible } \\
\text { P-Ti-Fe-rich melt }\end{array}$ & 1 \\
\hline Fedorivka & $\begin{array}{l}\text { Korosten pluton, } \\
\text { Ukraine }\end{array}$ & $\begin{array}{l}\text { Layered intrusion in the } \\
\text { Volodarsk-Volynskyy } \\
\text { massive anorthosite }\end{array}$ & $\begin{array}{l}\text { Fe-rich } \\
\text { olivine-gabbro }\end{array}$ & $\begin{array}{l}\text { Plag }\left(\mathrm{An}_{39-42}\right)+\mathrm{ol}\left(\mathrm{Fo}_{32-42}\right)+\text { augite } \\
\left(\mathrm{En}_{29-35} \mathrm{Fs}_{24-29} \mathrm{Wo}_{42-44}\right)+\mathrm{Fe}-\mathrm{Ti} \text { oxides }+ \text { apatite }\end{array}$ & $\begin{array}{l}\text { V-rich Ti-magnetite }\left(\text { Usp }_{52-78} ; \mathrm{V} \text { : }\right. \\
0.3-1.9 \% ; \mathrm{Cr}<0.01 \%)+ \text { ilmenite } \\
\left(\mathrm{Hem}_{1-6} ; \mathrm{MgO}: 0.5 \%\right)\end{array}$ & $\begin{array}{l}\text { Cumulates from a } \\
\text { ferrodioritic melt }\end{array}$ & 9 \\
\hline Suwalki & $\begin{array}{l}\text { Mazury complex, NE } \\
\text { Poland }\end{array}$ & $\begin{array}{l}\text { Parallel layers and lenses of } \\
\text { ore in massive anorthosite }\end{array}$ & $\begin{array}{l}\text { From Fe-Ti ore-rich } \\
\text { norite to norite }\end{array}$ & $\begin{array}{l}\text { Fe-Ti oxides }+ \text { plag }\left(\mathrm{An}_{43-60}\right)+\text { opx } \\
\left(\mathrm{En}_{60-77}\right)+\text { Al-spinel } \pm \mathrm{cpx} \pm \text { ol } \\
\left(\mathrm{Fo}_{65-75}\right) \pm \text { apatite }\end{array}$ & $\begin{array}{l}\text { Ti-magnetite }\left(\text { Usp }_{0-24} ; \mathrm{V}:\right. \\
0.20-0.67 \% ; \mathrm{Cr}: \\
0.02-0.53 \%)+ \text { ilmenite }\left(\mathrm{Hem}_{1-8} \text {; }\right. \\
\text { MgO: } 0.6-3.6 \%)\end{array}$ & $\begin{array}{l}\text { Cumulate enriched by } \\
\text { crystal sorting during } \\
\text { polybaric crystallization }\end{array}$ & 12 \\
\hline
\end{tabular}




\begin{tabular}{|c|c|c|c|c|c|c|c|}
\hline Deposit name & Location & Setting & Rock type & Main paragenesis & Oxide composition & Suggested origin & References \\
\hline Lac Tio & $\begin{array}{l}\text { Havre St Pierre } \\
\text { anorthosite, Grenville } \\
\text { Province, Quebec }\end{array}$ & $\begin{array}{l}\text { Funnel-shape intrusion of } \\
\text { massive ore in anorthosite }\end{array}$ & $\begin{array}{l}\text { Ilmenitite to } \\
\text { ilmenite-rich norite }\end{array}$ & $\begin{array}{l}\text { Ilmenite }+ \text { plag }\left({ }_{\text {An45-50 }}\right)+\text { Al-spinel }+ \text { opx } \\
\left(\text { En }_{58-76}\right)\end{array}$ & $\begin{array}{l}\text { Cr-, V-, Mg-rich hemo-ilmenite } \\
\text { (Hem } 23-29 ; \text { MgO: } 1.6-3.2 \% ; \mathrm{V} \\
0.18-0.24 \% \text {; Cr: } 0.05-0.23 \%)\end{array}$ & $\begin{array}{l}\text { Cumulate within a magma } \\
\text { conduit. differentiated } \\
\text { from a ferrodioritic melt }\end{array}$ & 10,17 \\
\hline Grader & $\begin{array}{l}\text { Havre St Pierre } \\
\text { anorthosite, Grenville } \\
\text { Province, Quebec }\end{array}$ & $\begin{array}{l}\text { Layered intrusion in } \\
\text { massive anorthosite }\end{array}$ & $\begin{array}{l}\text { Oxide apatite } \\
\text { (gabbro)norite } \\
\text { and massive } \\
\text { oxide layers }\end{array}$ & $\begin{array}{l}\text { Plag }\left(\mathrm{An}_{46-49}\right)+\text { ilmenite } \pm \text { magnetite } \pm \mathrm{opx} \\
\left(\mathrm{En}_{65-67}\right) \pm \text { apatite } \pm \mathrm{cpx}\end{array}$ & $\begin{array}{l}\text { Mg-poor hemo-ilmenite }\left(\mathrm{Hem}_{20-32} \text {; }\right. \\
\text { MgO: 0.9-2.5\%; V: 0.11-0.23\%; Cr: } \\
\text { 0.001-0.087\%) + magnetite (Usp } 1 \text {; } \\
\text { V: } 0.24 \% \text {; Cr: 0.006-0.032\%) }\end{array}$ & $\begin{array}{l}\text { Cumulate enriched by } \\
\text { crystal sorting in a } \\
\text { ferrodioritic liquid }\end{array}$ & 11 \\
\hline Big Island & $\begin{array}{l}\text { Havre St Pierre } \\
\text { anorthosite, Grenville } \\
\text { Province, Quebec }\end{array}$ & $\begin{array}{l}\text { Massive Fe-Ti oxide dyke } \\
\text { crosscutting anorthosite }\end{array}$ & Ilmenitite & Ilmenite + rutile + sapphirine \pm plag & $\begin{array}{l}\text { Hemo-ilmenite }\left(\mathrm{Hem}_{20-30} ; \mathrm{MgO}:\right. \\
\text { 1.9-3.0\%; Cr: } 0.04-0.12 \% ; \mathrm{V}: \\
0.2 \%) \text { + rutile }\end{array}$ & Accumulation in a conduit & 13,21 \\
\hline St Urbain & $\begin{array}{l}\text { Grenville Province, } \\
\text { Quebec }\end{array}$ & $\begin{array}{l}\text { Lenses of massive ore along } \\
\text { foliation planes in } \\
\text { anorthosite }\end{array}$ & $\begin{array}{l}\text { Ilmenitite, with } \\
\text { local nelsonite }\end{array}$ & Ilmenite \pm rutile + biotite + sulfides + sapphirine & $\begin{array}{l}\text { Hemo-ilmenite }\left(\mathrm{Hem}_{11-27} ; \mathrm{MgO}:\right. \\
2.2-3.7 \% ; \mathrm{Cr}: 0.05-0.12 \% ; \mathrm{V}: \\
0.17-0.24 \%)+ \text { rutile }\end{array}$ & $\begin{array}{l}\text { Accumulation at the base } \\
\text { of a magma chamber }\end{array}$ & 13,21 \\
\hline Desgrosbois & $\begin{array}{l}\text { Grenville Province, } \\
\text { Quebec }\end{array}$ & $\begin{array}{l}\text { Irregular intrusion of } \mathrm{Fe}-\mathrm{Ti} \\
\text { oxide rich rock in a } \\
\text { gabbroic anorthosite }\end{array}$ & $\begin{array}{l}\text { Fe-Ti oxide rich } \\
\text { gabbro }\end{array}$ & Fe-Ti oxides + opx + cpx + Al-spinel \pm apatite & Ti-magnetite + ilmenite & Late stage & 17 \\
\hline Ivry & $\begin{array}{l}\text { Grenville Province, } \\
\text { Quebec }\end{array}$ & $\begin{array}{l}\text { Steeply-dipping lenses of } \\
\text { massive ore in massive } \\
\text { anorthosite }\end{array}$ & Ilmenitite & Ilmenite + sulfides + Al-spinel & $\begin{array}{l}\text { Similar to Allard Lake deposit: } \\
\text { hemo-ilmenite (Hem } \text { Hem; }_{33} \text { Mg: } \\
\text { 1-2.4\%; V: } 0.3-0.8 \% ; \mathrm{Cr}: 0.1 \%)\end{array}$ & & 18,19 \\
\hline Lac De La Blache & $\begin{array}{l}\text { La Blache anorthosite, } \\
\text { Grenville Province, } \\
\text { Quebec }\end{array}$ & $\begin{array}{l}\text { Steeply dipping tabular } \\
\text { bodies in anorthosite }\end{array}$ & Magnetitite & Fe-Ti oxides + Al-spinel + ol $\left(\mathrm{Fo}_{61}\right)$ & $\begin{array}{l}\text { Ti-magnetite }\left(\text { Usp }_{55-69} ; \mathrm{MgO}:\right. \\
2.5-4.7 \% ; \mathrm{V}: 0.26 \%) \pm \text { ilmenite } \\
\left(\mathrm{Hem}_{66}\right)\end{array}$ & $\begin{array}{l}\text { Probably cumulate } \\
\text { enriched by crystal sorting }\end{array}$ & $17,20,21$ \\
\hline Iron Mountain & $\begin{array}{l}\text { Laramie anorthosite } \\
\text { complex, Wyoming }\end{array}$ & $\begin{array}{l}\text { Tabular bodies gradational } \\
\text { with an Fe-rich } \\
\text { leucotroctolite intruding an } \\
\text { anorthosite dome }\end{array}$ & $\begin{array}{l}\text { Pure Fe-Ti } \\
\text { oxides Fe-Ti-rich } \\
\text { olivine gabbro } \\
\text { and troctolite }\end{array}$ & Fe-Ti oxides \pm olivine \pm plag \pm cpx & $\begin{array}{l}\text { Ti-magnetite }\left(\mathrm{Usp}_{35} ; \mathrm{V}: 0.75 \% \text {; Cr: }\right. \\
0.14 \%)+\mathrm{Ilm}\left(\mathrm{Hem}_{4} ; \mathrm{MgO}: 2.6 \% ; \mathrm{V}:\right. \\
0.03 \% ; \mathrm{Cr}<0.006 \%)\end{array}$ & $\begin{array}{l}\text { Crystallized from } \\
\text { Fe-Ti-P-rich silicate } \\
\text { liquids and emplaced as } \\
\text { crystal mushes or in the } \\
\text { solid state }\end{array}$ & $14,14 \mathrm{~b}, 15$ \\
\hline Sybille & $\begin{array}{l}\text { Laramie anorthosite } \\
\text { complex, Wyoming }\end{array}$ & $\begin{array}{l}\text { Ore bodies gradational } \\
\text { with an Fe-rich } \\
\text { leucotroctolite intruding an } \\
\text { anorthosite dome }\end{array}$ & Fe-Ti oxide bodies & $\begin{array}{l}\text { Fe-Ti oxides + graphite }(0.5-1 \%)+\text { apatite } \\
(<1-70 \%)+\text { ol }\left(\mathrm{Fo}_{40-60}\right)+\text { plag }\left(\mathrm{An}_{45-98}\right)\end{array}$ & $\begin{array}{l}\text { Possibly a Ti-magnetite } \\
\left(\text { Usp }_{80} \mathrm{Mt}_{15} \mathrm{Herc}_{5}\right) \text { that resulted in } \\
\text { low- to medium } \mathrm{Ti} \\
\text { magnetite }+ \text { interstitial } \\
\text { ilmenite }+ \text { spinel }\end{array}$ & $\begin{array}{l}\text { Crystallized from } \\
\text { Fe-Ti-P-rich silicate liquids } \\
\text { and emplaced as crystal } \\
\text { mushes or in the solid state }\end{array}$ & 15,16 \\
\hline Sanford Lake & $\begin{array}{l}\text { Marcy anorthosite, } \\
\text { Adirondacks, New York }\end{array}$ & $\begin{array}{l}\text { Concordant (ultra)mafic } \\
\text { layers and cross-cutting } \\
\text { dykes in anorthosite }\end{array}$ & $\begin{array}{l}\text { Oxide-rich } \\
\text { metagabbro }\end{array}$ & $\begin{array}{l}\text { Fe-Ti oxides }+ \text { plag } \\
\left(\mathrm{An}_{24-47}\right)+\text { augite }+ \text { opx/Fe-rich } \\
\text { ol }+ \text { apatite }+ \text { garnet }\end{array}$ & $\begin{array}{l}\text { Ilmenite (Hem } 2-8 ; \text { MgO: } 0.9-2.3 \% ; \mathrm{Cr} \text { : } \\
\text { 0.01-0.1\%; V: } \\
\text { 0.09-0.4\%) + magnetite (Uspo.2-10; } \\
\text { Cr: 0.25-2.6\%; V: 0.8-2\%) }\end{array}$ & $\begin{array}{l}\text { Metamorphosed cumulates } \\
\text { and late-stage liquids } \\
\text { (dykes) }\end{array}$ & 22 \\
\hline
\end{tabular}

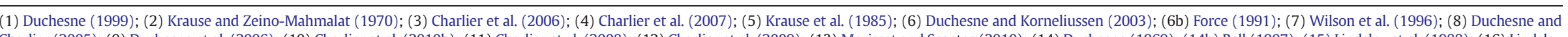

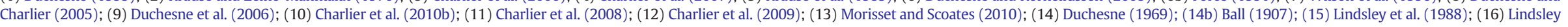
(2003); (17) Corriveau et al. (2007); (18) Rose (1969); (19) Schrijver (pers. comm.); (20) Anderson and Morin (1969); (21) Perreault and Hébert (2003); (22) Ashwal (1982). 


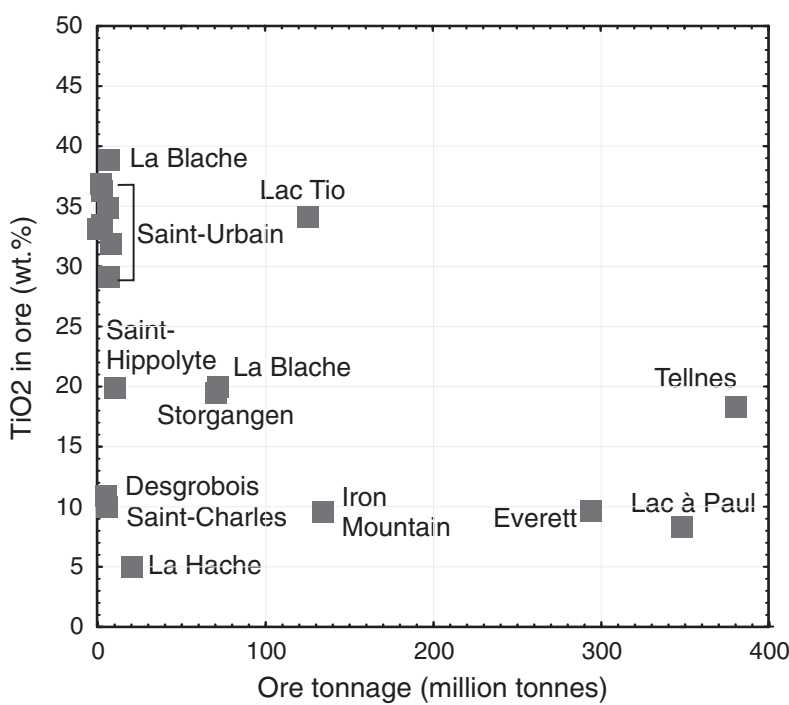

Fig. 4. $\mathrm{TiO}_{2}$ content in ore rocks (wt.\%) as a function of estimate reserves of ore tonnage (in millions of metric tons) for selected iron-titanium deposits. Data compiled by Woodruff et al. (2013).

and Charlier, 2005). The Grader intrusion in the Havre-Saint-Pierre anorthosite (Charlier et al., 2008), the Fedorivka intrusion in the Korosten Complex (Duchesne et al., 2006), and the Bøstølen intrusion in the Rogaland Anorthosite Province (Krause et al., 1985) are other examples. Melanocratic and leucocratic rocks alternate at various scales ( $\mathrm{cm}$ - to decameter), producing highly irregular patterns in the grade of the ore. The rocks are thus characterized by cumulate textures with abrupt changes in modal proportions.

\subsection{Massive to layered tabular bodies}

Some ore bodies in anorthosite plutons take the form of sills, some tens of meters thick but extending for several kilometers. They can be parallel to the foliation of the anorthosite and can be deformed. They have sharp contacts with the host anorthosite and may contain large anorthosite blocks. A major example is the Storgangen intrusion in SW Norway, which is ca. $4 \mathrm{~km}$ long and a few meters to $50-60 \mathrm{~m}$ thick (Schiellerup et al., 2003). Rocks are strongly modally-layered and massive ilmenite occurs at the footwall contact. Upsection cryptic layering is marked by evolving plagioclase composition and decreasing $\mathrm{Cr}$ concentration in Fe-Ti oxides. The Tellnes deposit can also be thought of as a massive, tabular and deformed igneous body. It is extended on both sides for ca. $15 \mathrm{~km}$ by a 5 to 10 m-thick dyke which varies in composition from jotunite to quartz mangerite (Wilmart et al., 1989). The contact is also sharp between the massive ilmenitite and the host anorthosite of the Big Island dyke (Morisset et al., 2010). This structure is $30 \mathrm{~m}$ wide by $250 \mathrm{~m}$ long, with no systematic compositional or mineralogical zoning along or perpendicular to strike.

In these structures, accumulation of oxide minerals in the lower part of a dyke has been invoked (Charlier et al., 2007; Morisset et al., 2010). It has been suggested that the upper, eroded part of the dyke should contain most of the co-crystallizing plagioclase ( \pm orthopyroxene and olivine), or that plagioclase was transported laterally away through the conduit system.

\subsection{Lenticular ore bodies}

Ore bodies intimately associated with the host rock are observed locally in anorthosite plutons. The ore rocks do not represent welldefined discordant intrusions but instead display gradation into the host rocks. In the Suwalki anorthosite, individual ore bodies with morphologies of lenses, veins or layers are common (Charlier et al.,
2009). Their orientations are parallel to the foliation and margins of the anorthosite. Lenticular bodies are commonly deformed. The Blåfjell deposit (Krause and Zeino-Mahmalat, 1970), minor deposits in the Håland-Helleren and Åna-Sira anorthosites of the Rogaland anorthosite province (Duchesne, 1999), and the major Damiao deposit in China (Chen et al., 2013) can also be classified as lenticular ore bodies.

\section{Sequence of crystallization in layered intrusions}

The stratigraphic evolution of cumulus assemblages in layered intrusions associated with massif-type anorthosite provides information about the formation of Fe-Ti oxide ores and the genetic relationships among different types of ore. The crystallization sequence produced during cooling of the parental magma is recorded in the successive appearance of liquidus phases in intrusions with well-defined stratigraphy (Fig. 5).

The Layered Series of the Bjerkreim-Sokndal layered intrusion is subdivided into five megacyclic units (MCUs), the base of each displaying a compositional reversal to more primitive mineral compositions as a result of a new magma influx (Duchesne, 1972; Jensen et al., 1993; Wilson et al., 1996). Each MCU is subdivided into zones according to cumulus mineral assemblages. Plagioclase is the first cumulus mineral to appear and is present throughout the entire sequence. It is followed by ilmenite, which is shortly followed by Ca-poor pyroxene. Magnetite precedes apatite and Ca-rich pyroxene, which appear simultaneously. At the top of MCU IV, hypersthene is replaced by inverted pigeonite. At the base of MCUs III and IV, the magma crystallizes olivine and magnetite instead of hypersthene.

In the Fedorivka layered intrusion, the dominant cumulus assemblage is plagioclase $\left(\mathrm{An}_{39-42}\right)$, iron-rich olivine $\left(\mathrm{Fo}_{32-42}\right)$, augite $\left(\mathrm{En}_{29-35} \mathrm{Fs}_{24-29} \mathrm{Wo}_{42-44}\right)$, ilmenite $\left(\mathrm{Hem}_{1-6}\right)$, and then both Ti-magnetite (Usp $52-78$ ) and apatite (Duchesne et al., 2006) appear simultaneously.

The Grader intrusion in the Havre-Saint-Pierre anorthosite has a distinctive crystallization sequence: plagioclase and ilmenite are followed by apatite, then orthopyroxene together with magnetite, and finally clinopyroxene. This atypical sequence of crystallization resulted in the formation of plagioclase-ilmenite-apatite cumulates or nelsonites in plagioclase-free layers (Charlier et al., 2008).

In all these examples, plagioclase is always on the liquidus. Orthopyroxene (or locally olivine) is usually the second liquidus phase followed by ilmenite. In some intrusions, ilmenite appears before

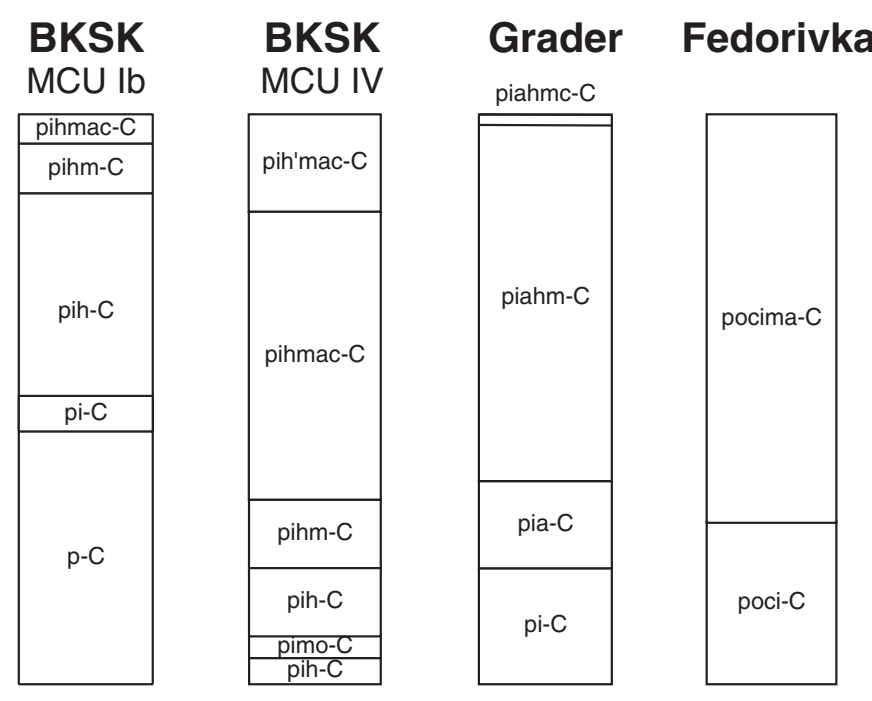

Fig. 5. Comparative stratigraphy and crystallization sequences in the Grader layered intrusion (Charlier et al., 2008), the Bjerkreim-Sokndal layered intrusion (Wilson et al., 1996) and the Fedorivka layered intrusion (Duchesne et al., 2006). Mineral abbreviations: $\mathrm{p}=$ plagioclase; $\mathrm{h}=$ orthopyroxene; $\mathrm{h}^{\prime}=$ inverted pigeonite; $\mathrm{o}=$ olivine; $\mathrm{i}=$ ilmenite; $\mathrm{m}=$ magnetite; $\mathrm{a}=$ apatite; $\mathrm{c}=$ clinopyroxene; $-\mathrm{C}=$ cumulus. 

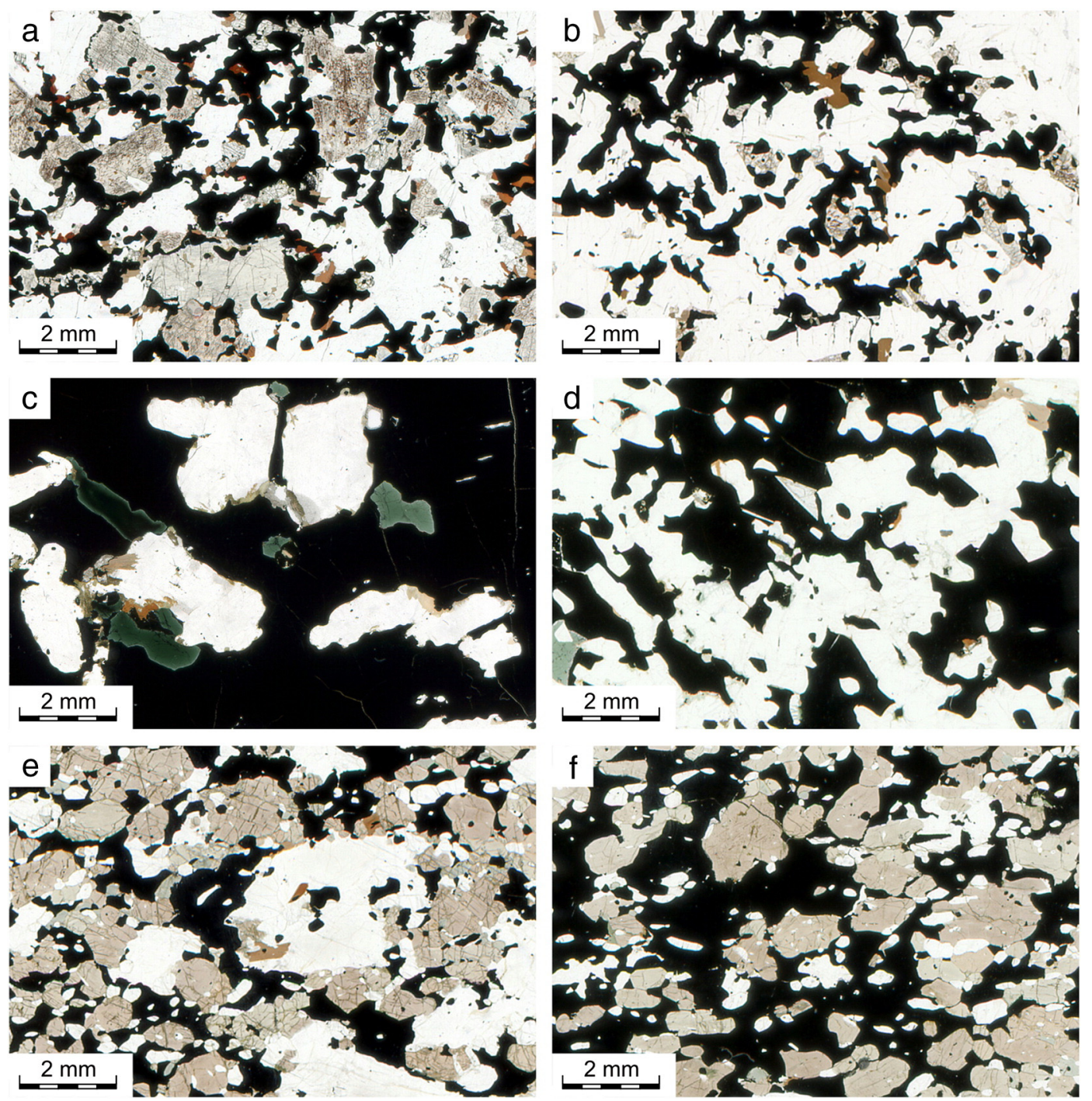

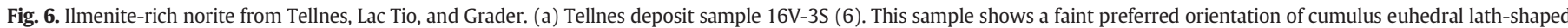

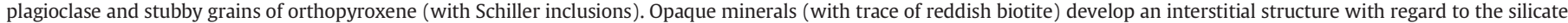

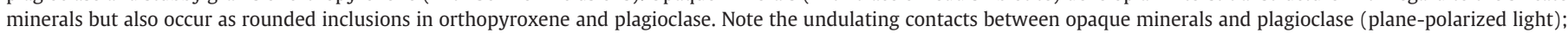

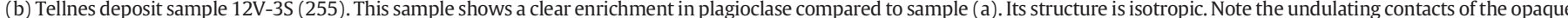

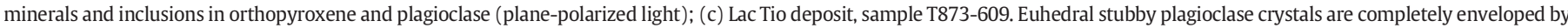

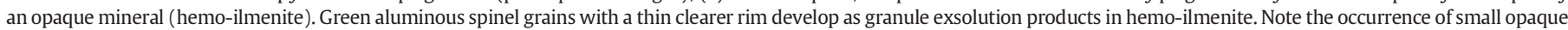

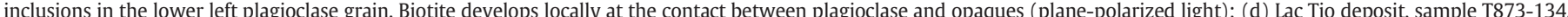

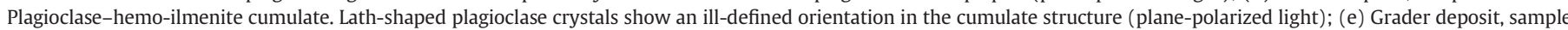

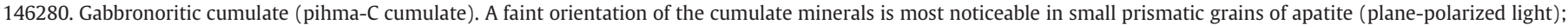

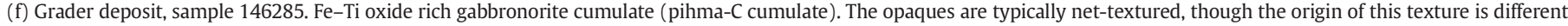

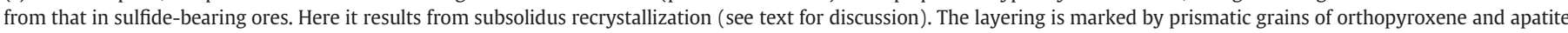
(plane-polarized light).

orthopyroxene. The early saturation of ilmenite, always before clinopyroxene, is characteristic of the evolution of magmas related to anorthosite provinces. Liquidus ilmenite also normally precedes the appearance of liquidus magnetite. Differences between the stratigraphic sequences presented in Fig. 5 are related to parent magma compositions, $\mathrm{fO}_{2}$ and degree of differentiation. For example, the most primitive cumulates in the Fedorivka intrusion are clearly more evolved than in other intrusions.

\section{A mineralogical classification for Fe-Ti-V-P deposits}

Several classification schemes have been proposed for $\mathrm{Fe}-\mathrm{Ti}$ deposits. Most of them stem from the observation of two types of deposits in Grenville anorthosites (Anderson and Morin, 1969). The first type is dominated by hemo-ilmenite and occurs in andesine anorthosite; the second type has titanomagnetite as the principal mineral and is found in labradorite anorthosite. Gross (1996) has proposed a similar division of the magmatic $\mathrm{Fe}-\mathrm{Ti} \pm \mathrm{V}$ deposits based on the principal ore minerals, with emphasis on the petrology and structure of the host intrusion. The first type is mainly made up of ilmenite or hemo-ilmenite with minor magnetite, and forms massive irregular discordant bodies or layered intrusions hosted in massif anorthosites. The second type mainly consists of titaniferous magnetite and minor ilmenite forming complex Fe-Ti oxide mineral assemblages hosted in layered and/or massive intrusions of (leuco-)gabbros, norites and rocks of intermediate composition. Both types can contain resources of apatite and vanadium.

A study of Fe-Ti oxides in the Bjerkreim-Sokndal layered intrusion (Duchesne, 1972) showed a continuous evolution of ore composition, starting with hemo-ilmenite as the only oxide in the lower part of the 

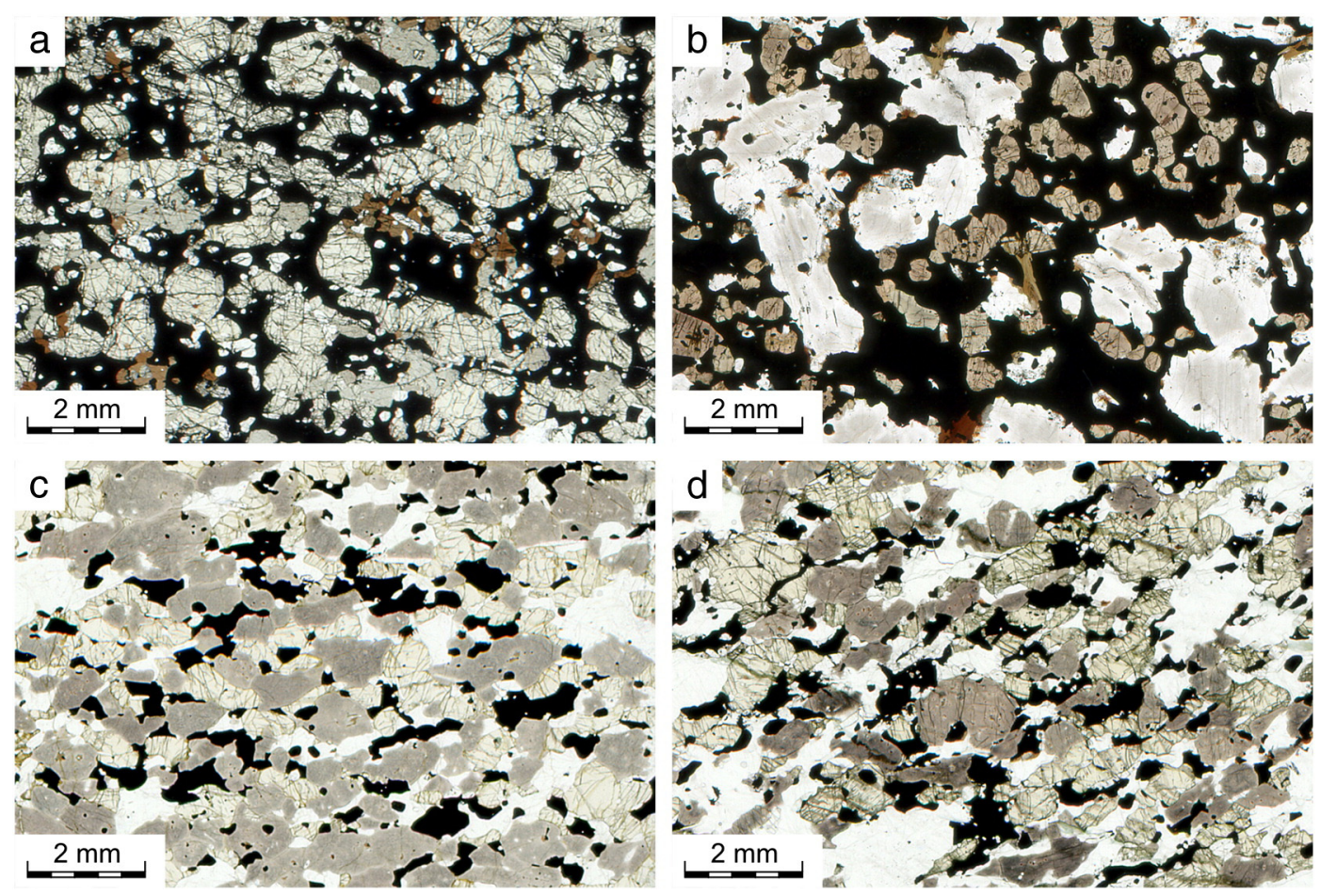

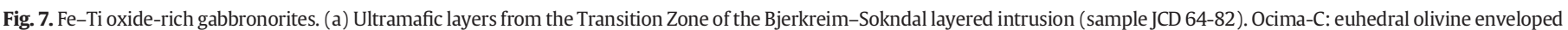

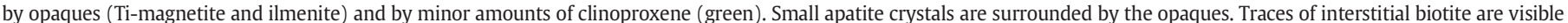

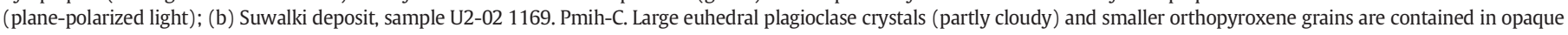

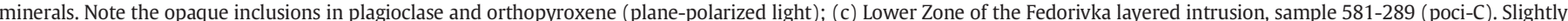

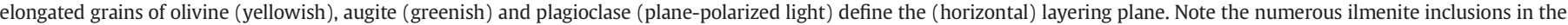

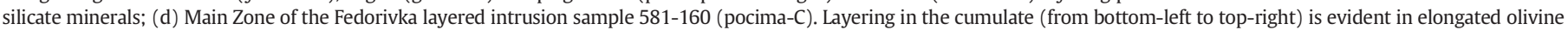

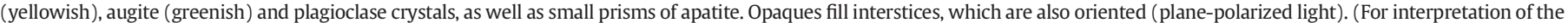
references to color in this figure legend, the reader is referred to the web version of this article.)

macrocyclic units, joined upsection by Ti-poor magnetite, followed by a Ti-magnetite + ilmenite + apatite assemblage in the upper part of the sequence. Both oxides display continuous variation in composition and relative abundance and are associated with andesine. This succession of parageneses was interpreted as a simple fractional crystallization process without being necessarily linked to an andesine or labradorite plagioclase (Duchesne, 1972), which defies the simple classification based on the anorthosite type (andesine vs. labradorite). The following classification was therefore adopted for the Rogaland deposits (Duchesne, 1973 ) on the basis of the principal minerals: Type I: hemo-ilmenite alone; Type II: (hemo)-ilmenite + Ti-poor magnetite; and Type III: Timagnetite + homogeneous ilmenite + apatite, including nelsonite.

Studies of the Lac St. Jean anorthosite suite support the suggestion that titanomagnetite deposits are exclusively found in labradorite anorthosites and are usually devoid of apatite (Perreault, 2003; Hébert et al., 2005). Conversely, andesine anorthosites contain ilmenite + magnetite \pm apatite, and include nelsonite or oxide-rich apatite gabbronorite. This is however not generally true. At Laramie (Wyoming), the Sybille deposit is found in an andesine anorthosite (Poe Mountain; Scoates et al., 2010); while the mainly similar Iron Mountain deposit occurs in a labradorite anorthosite (Chugwater; Lindsley et al., 2010).

Finally, Corriveau et al. (2007) distinguish a first type of disseminated $\mathrm{Fe}-\mathrm{Ti}$ oxides ore from a second type of massive bodies. They also constitute a distinct third type with nelsonite, a fourth type with late injections of magnetitite, ilmenitite and oxide-rich norite, and a fifth type with magnetite-bearing acidic dykes cutting anorthosites (without economic potential).

We propose here a classification that separates ilmenite and $\mathrm{Ti}$ magnetite deposits but does not preclude the existence of transitional types. We do not distinguish between massive, disseminated and layered occurrences. We define nelsonite as a third deposit type although apatite can be found in the first two types. We separate nelsonites from other ore types because the process yielding an $\mathrm{Fe}-\mathrm{Ti}$ oxide + apatite assemblage in the conspicuous absence of silicates requires very specific conditions. Finally, we propose a fourth type: the rare ilmenite + rutile association, which has great potential economic value.

Table 2 summarizes the principal characteristics of representative Proterozoic ore deposits. It includes the composition of the accompanying minerals and the composition of the Fe-Ti oxide minerals. It also reports the various genetic processes that have been proposed in the literature.

\section{1. (Gabbro-)noritic ilmenite ore \pm apatite \pm magnetite}

This type of ores includes the Tellnes deposit and the Lac Tio Mine (Fig. 6). The ore is dominated by ilmenite containing variable amount of hematite exsolutions. Accompanying phases are plagioclase, \pm orthopyroxene, \pm olivine, \pm clinopyroxene, \pm magnetite \pm apatite. One can distinguish two sub-types: massive or layered hemo-ilmenite without magnetite, and (hemo)-ilmenite \pm magnetite \pm apatite.

Other localities with potential economic value occur in the Rogaland anorthosite province: Storgangen, Bøstølen, and Blåfjell (Krause and Zeino-Mahmalat, 1970; Krause and Pape, 1977; Krause et al., 1985; Duchesne, 1999; Schiellerup et al., 2003). Combined with P-resources, the upper part of MCU IV of the Bjerkreim-Sokndal layered intrusion (Wilson et al., 1996) and the Grader layered intrusion (Charlier et al., 2008) are of interest.

\subsection{Ti-magnetite- \pm apatite-dominated ore}

The distinction of this ore type is necessary from an economic perspective because Ti from Ti-magnetite cannot presently be economically 


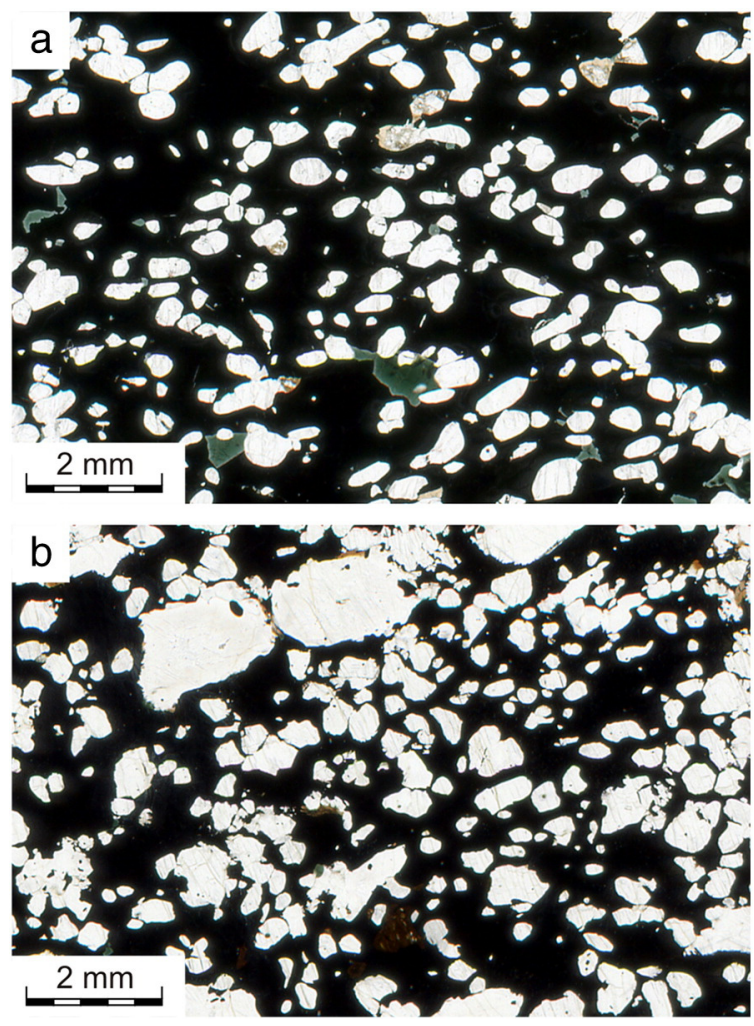

Fig. 8. Nelsonites (a) Kydlansvatn deposit (Rogaland), sample 79-19-1B. Hemo-ilmenite contains euhedral grains of apatite and granules of green aluminous spinel with thin clear rims (plane-polarized light); (b) Grader deposit, sample 146290 (pia-C). The layering is defined by small apatite grains, embedded in ilmenite. Note two larger stubby plagioclase crystals, also parallel to this plane (plane-polarized light).

recovered. However, the magnetite may represent a significant source of $\mathrm{V}$, so the economic potential has to be evaluated, especially when combined with Ti from ilmenite and P from apatite (Fig. 7). Examples of this ore type are present in the Magpie Mountain (Lac St Jean anorthosite, Québec; Perreault, 2003), the Suwalki anorthosite (NE Poland; Charlier et al., 2009), the Fedorivka layered intrusion (Korosten Complex, Ukraine; Duchesne et al., 2006), the Krapivnia deposit (Korosten Complex, Ukraine; Gursky et al., 2003) and in the Transition Zone of the Bjerkreim-Sokndal layered intrusion (Duchesne et al., 1987).

\subsection{Nelsonite}

Nelsonites are Fe-Ti-oxide-apatite rocks (Fig. 8). The term was initially introduced by Watson and Taber (1910) for rutile-apatite and (hemo-)ilmenite-(Ti-)magnetite-apatite rocks, located in the Roseland andesine (antiperthitic) anorthosite in Virginia (Owens and Dymek, 1999). Unlike oxide-apatite gabbronorites (Owens and Dymek, 1992) these rocks are devoid of silicates. Some nelsonites display modal proportions close to one third apatite, two thirds Fe-Ti oxide (Philpotts, 1967; Kolker, 1982) but this is not a general rule. Apatite is commonly fluorine-rich (Dymek and Owens, 2001) and REE-rich (Duchesne, 1999).

\subsection{Rutile-ilmenite ore}

Two localities associated with massif-type anorthosite are known for their rutile-ilmenite associations: several occurrences in the Saint-Urbain anorthosite, and the Big Island ore deposit in the Havre Saint-Pierre anorthosite (Warren, 1912; Morisset and Scoates, 2008; Morisset et al., 2009, 2010). This type of ore may also display an unusual association with sapphirine (Dymek, 1984), which is found in close

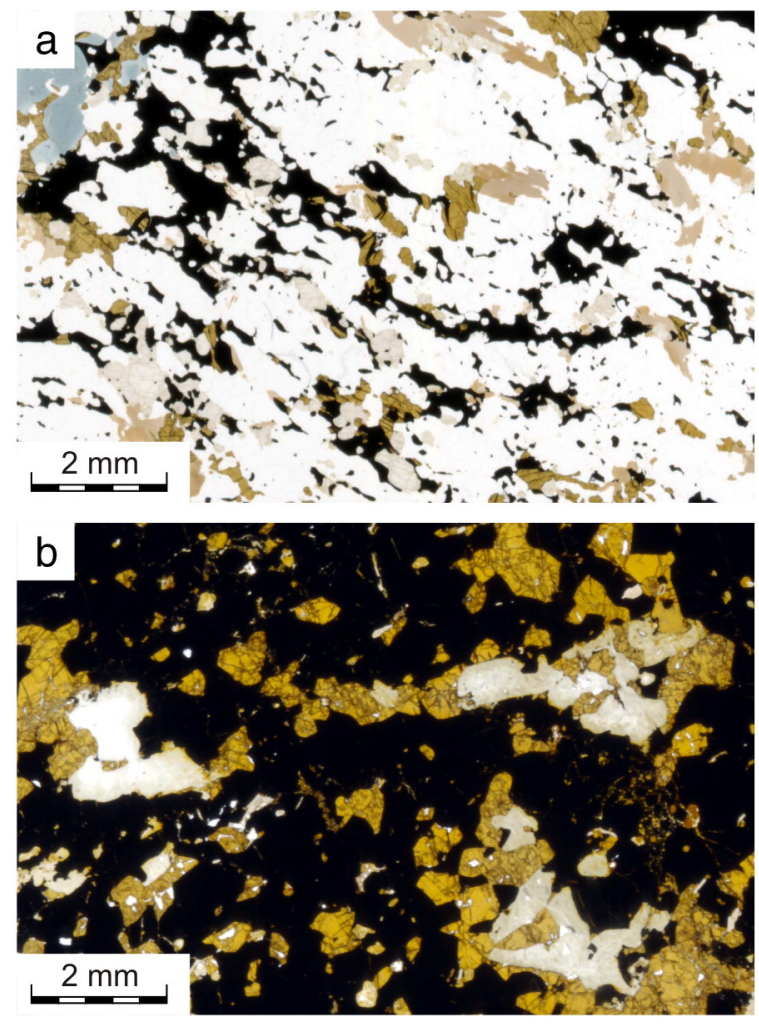

Fig. 9. Rutile-bearing ore (a) Saint Urbain, Coulomb East, sample 2015-A4: leuconorite with ilmenite and rutile grains defining the foliation (plane-polarized light). The blue phase in the upper left corner is sapphirine; (b) Saint Urbain, General Electric, sample 2030-C4: euhedral crystals of rutile and plagioclase wrapped in an ilmenite matrix (plane-polarized light) (samples from Morisset et al., 2010). (For interpretation of the references to color in this figure legend, the reader is referred to the web version of this article.)

association with pleonaste (Fig. 9). At Saint-Urbain and Big Island, both rutile and sapphirine occur with hemo-ilmenite, plagioclase $\left(\mathrm{An}_{39-51}\right)$, high-Al orthopyroxene (5.2-9.1 wt.\% $\left.\mathrm{Al}_{2} \mathrm{O}_{3}\right)$, and aluminous spinel, with trace amounts of apatite, corundum and sulfide minerals. In these rocks, sapphirine is interpreted as forming by subsolidus reactions (e.g., hercynite + orthopyroxene + rutile \pm corundum $=$ sapphirine + ilmenite). Morisset et al. (2010) interpret both ilmenite and the larger rutile crystals to be magmatic phases that segregated and accumulated by gravitational settling from Fe-Ti-enriched residual magmas (ferrobasaltic, jotunitic) following crystallization of the host anorthosite. There are, however, no experimental data supporting a magmatic origin for rutile. Small lenses of rutile (10 to $200 \mu \mathrm{m}$ thick) within ilmenite and surrounded by hematite are related to late oxidation.

\section{The stability of ilmenite and magnetite}

\section{1. $\mathrm{fO}_{2}$ and parental magma composition}

Since the work of Buddington and Lindsley (1964) experimental studies have confirmed the strong influence of oxygen fugacity on the stability fields of Fe-Ti oxides (Hill and Roeder, 1973; Grove and Baker, 1984; Juster et al., 1989; Snyder et al., 1993; Toplis and Carroll, 1995). These one-atmosphere experiments on ferrobasalts show that the stability field of magnetite expands with increasing $\mathrm{fO}_{2}$. Consequently, at high $\mathrm{fO}_{2}$, the appearance of magnetite would precede that of ilmenite. However, Toplis and Carroll (1995) have shown that $\mathrm{fO}_{2}$ only affects the stability field of magnetite whereas the saturation point of ilmenite is controlled by the $\mathrm{TiO}_{2}$ content of the melt. 
Consequently, the larger stability field of ilmenite alone under reduced conditions is a consequence of magnetite destabilization, and thus iron-enrichment in the melt. On the other hand, even in oxidizing conditions, melts with a high $\mathrm{TiO}_{2}$ content might reach ilmenite saturation before magnetite, and in some cases may only crystallize hemo-ilmenite without magnetite (Lattard et al., 2005). This situation is observed in the Grader layered intrusion (Charlier et al., 2008), where the high hematite content in ilmenite is an indicator of the high oxygen fugacity in the melt (ca. NNO +1 ). Ilmenite is the first liquidus Fe-Ti oxide and remains modally more abundant than magnetite when this mineral joins the crystallizing assemblage. The early saturation of hematiterich ilmenite instead of Ti-magnetite in the Grader intrusion is thus controlled by the high $\mathrm{TiO}_{2}$ content of the parental magma.

\subsection{The role of polybaric crystallization}

The dynamic and polybaric crystallization of massif-type anorthosites may have a significant influence on the formation of Fe-Ti oxide ores. The ascent of the anorthosite mush may mechanically sort disseminated crystals of Fe-Ti oxides, while the phase equilibria of the melt depend strongly on the crystallization pressure (Vander Auwera and Longhi, 1994).

Charlier et al. (2009) and Chen et al. (2013) have highlighted the role of polybaric crystallization in the formation of $\mathrm{Fe}$-Ti oxide ores in the Suwalki and Damiao anorthosites. These oxide ores crystallized continuously as the anorthosite diapir ascended, as suggested by several observations including variable $\mathrm{Al}_{2} \mathrm{O}_{3}$ content in orthopyroxene, which is related to the pressure of crystallization (Longhi et al., 1993). The crystallization of parental melts at varying pressure between 13 and 5 kbar also explains the different crystallization sequences observed in anorthosites and related rocks. Experimental liquidus phases of plausible parent magmas of massif-type anorthosite are initially saturated in plagioclase + orthopyroxene at 10-13 kbar (Fram and Longhi, 1992; Vander Auwera et al., 1998; Longhi et al., 1999). However, in many Fe-Ti oxide-rich rocks, plagioclase may occur with Fe-Ti oxides (ilmenite or ilmenite + magnetite) but without orthopyroxene. For example, in the Tellnes and Lac Tio deposits (Charlier et al., 2006, 2010b), orthopyroxene becomes a liquidus phase after plagioclase and ilmenite. As shown in high-Ti mare basalts from the Moon (Delano, 1980), it is more probable that the temperature-pressure slope for the orthopyroxene liquidus is much steeper than that for the ilmenite liquidus (Fig. 10). Consequently, although the assemblage plagioclase + high-alumina orthopyroxene crystallizes at high pressure (13-10 kbar), the assemblage plagioclase + ilmenite ( \pm magnetite) may crystallize at lower pressure (8-5 kbar) from the same parental magma. The occurrence of different cumulus assemblages may thus result from different pressures of crystallization.

\subsection{The role of $\mathrm{P}_{2} \mathrm{O}_{5}$}

The effects of phosphorus on differentiation of ferrobasalts have been investigated experimentally by Toplis et al. (1994a). $\mathrm{P}_{2} \mathrm{O}_{5}$ and FeO mutually enhance their solubilities due to formation of $\mathrm{Fe}^{3+}\left(\mathrm{PO}_{4}\right)^{3-}$ complexes in ferrobasaltic melts. The main role of $\mathrm{P}_{2} \mathrm{O}_{5}$ is thus to permit the formation of silicate melts with much higher $\mathrm{Fe}$ contents than is possible for P-poor compositions, allowing the concentration of Fe and Ti at higher levels. At a given temperature, the proportion of liquid and the modal plagioclase/pyroxene ratio increase with increasing $\mathrm{P}_{2} \mathrm{O}_{5}$ content. The addition of $\mathrm{P}_{2} \mathrm{O}_{5}$ to the melt decreases the abundance of augite and favors the crystallization of pigeonite. It also decreases the stability field of magnetite while the relative abundance of ilmenite remains approximately constant. The high $\mathrm{P}_{2} \mathrm{O}_{5}$ content of ferrodioritic or jotunitic liquids associated with anorthosites (e.g. Mitchell et al., 1996; Vander Auwera et al., 1998) is thus probably responsible for some characteristics of anorthosite suites, such as the

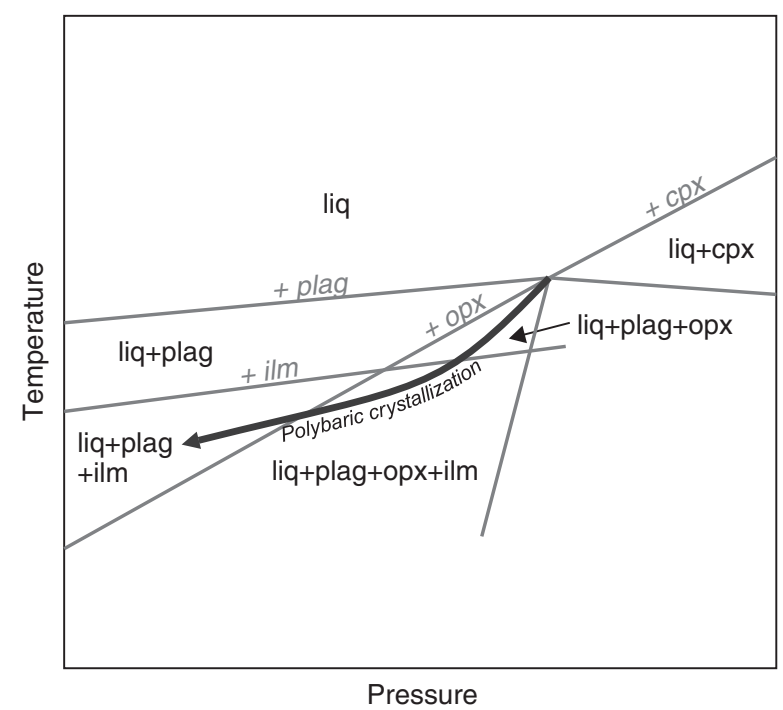

Fig. 10. Schematic phase equilibria in a pressure-temperature diagram showing the effect of pressure on the relative saturation of orthopyroxene and ilmenite.

leuconoritic character of rocks and the common early saturation of ilmenite compared to magnetite.

\section{Liquidus compositions of Fe-Ti oxides}

\subsection{Major elements}

Ilmenite and magnetite are members of two solid solutions (Fig. 11): the hematite-ilmenite series $\left(\mathrm{Fe}_{2} \mathrm{O}_{3}-\mathrm{FeTiO}_{3}\right)$ and the magnetiteulvöspinel series $\left(\mathrm{Fe}_{3} \mathrm{O}_{4}-\mathrm{Fe}_{2} \mathrm{TiO}_{4}\right)$. It has been shown that the magnetite content of magnetite-ulvöspinel series and the hematite content of ilmenite-hematite series both increase with increasingly oxidizing conditions (e.g. Buddington and Lindsley, 1964; Lattard et al., 2005; Sauerzapf et al., 2008). At given $\mathrm{fO}_{2}$ conditions, the magnetite content of magnetite decreases with increasing temperature. The ilmenite composition is less sensitive to temperature but its behavior is more complex due to the transition between the ordered $R \overline{3}$ structure and the disordered $R \overline{3} C$ structure. At oxygen fugacity conditions below $\mathrm{QFM}+2$ (QFM = the quartz-fayalite-magnetite $\mathrm{fO}_{2}$ buffer), the hematite-content of ilmenite increases with increasing temperature (Lattard et al., 2005). Conversely, at more oxidizing conditions, the hematite content of ilmenite decreases with increasing temperature.

The $\mathrm{Al}_{2} \mathrm{O}_{3}$ content in magnetite is positively correlated with the $\mathrm{Al}_{2} \mathrm{O}_{3}$ content of the melt with a crystal/melt partition coefficient of $\sim 0.3$. The $\mathrm{Al}_{2} \mathrm{O}_{3}$ content of ilmenite is generally very low ( $<0.5 \mathrm{wt} . \%$ ). $\mathrm{MnO}$ and $\mathrm{MgO}$ in magnetite and ilmenite are also a function of melt composition, with $\mathrm{MgO}$ partition coefficients $\sim 1$ for and $\mathrm{MnO}$ partition coefficients $\sim 2$ for both minerals. The equilibrium $\mathrm{MgO}$ content of the oxides is dependent on $\mathrm{fO}_{2}$, because it is dependent on $\mathrm{Fe}-\mathrm{Mg}$ exchange with the ferromagnesian silicates, which in turn are strongly dependent on $\mathrm{fO}_{2}$ (see QUILF reactions in Lindsley and Frost, 1992).

\subsection{Cr and V partitioning}

$\mathrm{Cr}$ and $\mathrm{V}$ are compatible in ilmenite and magnetite. However, experiments in ferrobasalts by Toplis and Corgne (2002) have shown that the partition coefficients of these two elements between magnetite and liquid $\left(D_{V}^{\mathrm{mt} / \text { /iq }}\right.$ and $\left.\mathrm{D}_{\mathrm{Cr}}^{\mathrm{mt} / \mathrm{liq}}\right)$ are closely related to oxygen fugacity $\left(f \mathrm{O}_{2}\right)$. In phosphorus-bearing systems, $\mathrm{D}_{\mathrm{Cr}}^{\mathrm{mt} / \mathrm{iq}}$ increases from 27 to 291 with decreasing oxygen fugacity from NNO +2.6 to NNO-0.7 (where NNO is the nickel-nickel oxide buffer), while $D_{V}^{\text {mt/liq }}$ increases from 2 


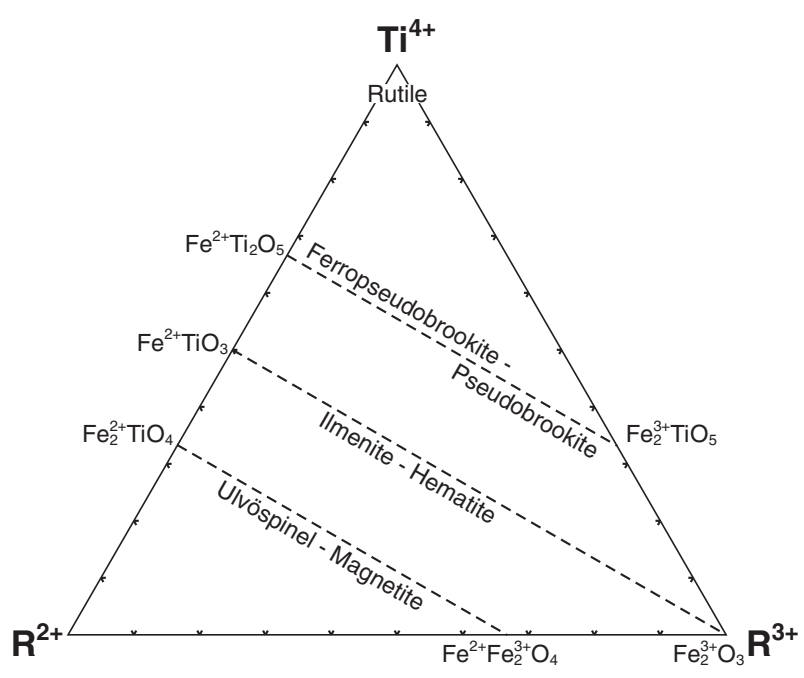

Fig. 11. Ternary plot of cation proportions of $\mathrm{R}^{2+}\left(\mathrm{Fe}^{2+}, \mathrm{Mg}, \mathrm{Mn}^{2+}\right.$, etc.), $\mathrm{R}^{3+}\left(\mathrm{Fe}^{3+}, \mathrm{Cr}^{3+}\right.$ $\mathrm{V}^{3+}, \mathrm{Al}^{3+}$, etc.), and $\mathrm{Ti}^{4+}$, showing the solid-solution series hematite-ilmenite, magnetiteulvöspinel, and pseudobrookite-ferropseudobrookite.

to 29. Consequently, even at high $\mathrm{fO}_{2}, \mathrm{Cr}$ is compatible in the bulk crystallizing assemblage $\left(D_{\mathrm{Cr}}^{\mathrm{mt} / \mathrm{iq}}>1\right)$, as long as the proportion of magnetite is more than $4 \%$. However, for relatively high $\mathrm{fO}_{2}$ and thus low $\mathrm{D}_{\mathrm{V}}^{\mathrm{mt} / \mathrm{iq}}, \mathrm{V}$ could behave overall as an incompatible element. The effect of $\mathrm{fO}_{2}$ on $\mathrm{D}_{\mathrm{V}}^{\mathrm{mt} / \mathrm{liq}}$ can explain the non-systematic relationship between $\mathrm{Cr}$ and $\mathrm{V}$ in various $\mathrm{Fe}-\mathrm{Ti}$ deposits. In the Fedorivka layered intrusion (Duchesne et al., 2006), low $\mathrm{fO}_{2}$ values are responsible for the late appearance of cumulus magnetite and the high $\mathrm{V}^{3+}$-content of the melt, reflected in the high $\mathrm{V}$-content of the initial liquidus magnetite (up to $1.85 \% \mathrm{~V}$ ). The high $\mathrm{fO}_{2}$ in the Suwalki anorthosite (Charlier et al., 2009) is responsible for the relatively low $\mathrm{V}$ content of magnetite (0.37-0.67 wt.\% V). The effect of $\mathrm{fO}_{2}$ also explains the low $\mathrm{V}$ concentration $(<0.5 \mathrm{wt} . \% \mathrm{~V})$ of magnetite from Fe-Ti oxide deposits in the Panxi region that crystallized under relatively high oxygen fugacity (Pang et al., 2010). Conversely, in the Bushveld complex, V in magnetite reaches $1.3 \mathrm{wt}$.\% at the base of magnetite layers that crystallized under more reducing conditions (Cawthorn and Molyneux, 1986).

\section{Postcumulus evolution of Fe-Ti oxide minerals}

\subsection{Re-equilibration with trapped liquid}

The crystallization of trapped liquid is known to have a strong influence on the composition of primary cumulus minerals (Barnes, 1986). However, because Fe-Ti oxides are strongly affected by subsolidus processes, the effect of trapped liquid is not easily deconvolved from other mechanisms. Expected zoning of cumulus phases is likely to be erased by later re-crystallization and intra-mineral diffusion.

The effect of trapped liquid on ilmenite composition in the Tellnes ilmenite deposit has been quantified by Charlier et al. (2007) using the compositions of cumulus and intercumulus ilmenite and their relative proportions. For compatible elements that are enriched in cumulus ilmenite, the trapped liquid mainly has a diluting effect because of its low concentrations of these elements. If the proportion of cumulus ilmenite is high compared to the amount of ilmenite crystallized from the trapped liquid, the influence of trapped liquid crystallization is minor. For industrial purposes, the crystallization of ilmenite from the trapped liquid usefully reduces the $\mathrm{Cr}$ content of ilmenite, as well as other compatible element contents. Incompatible elements are enriched in ilmenite crystallized from the trapped liquid, which in turn increases the concentrations of these elements in re-equilibrated ilmenite. However, trapped liquid crystallization has a negative effect on the grade of the ore: it tends to decrease the $\mathrm{TiO}_{2}$ content of the bulk rock because it is Ti-poorer than the cumulus assemblage.

\subsection{Re-equilibration with ferromagnesian silicates}

Re-equilibration with trapped liquid is prolonged during subsolidus evolution by reaction with other phases, particularly ferromagnesian silicates. The main effect of this re-equilibration is to drive down the
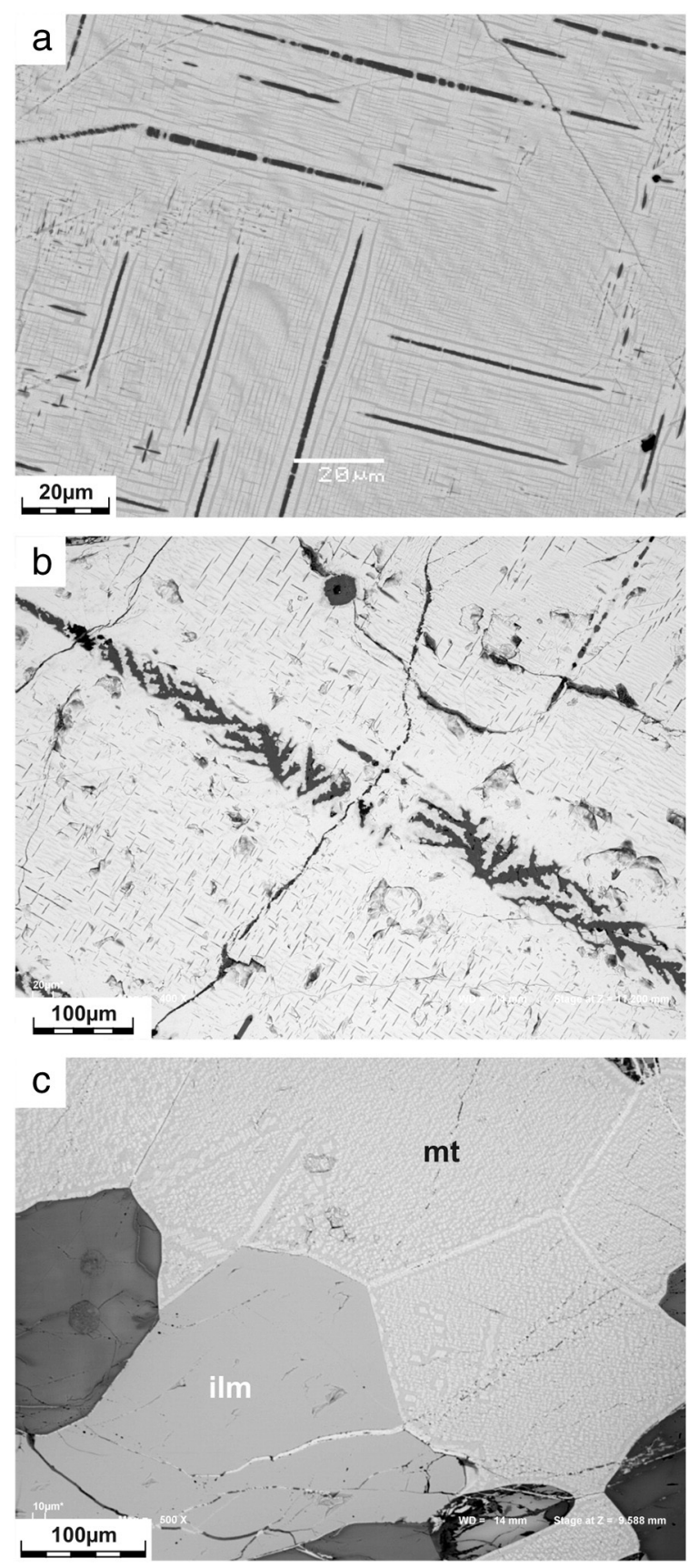

Fig. 12. Exsolutions in magnetite. (a) Cloth-textured magnetite with ulvöspinel and hercynitic spinel exsolutions in the $\{100\}$ planes of magnetite. Coarser ulvöspinel exsolutions around spinel lamellae define the "box-like" structures of Ramdohr (1980). As frequently observed, the ulvöspinel lamellae, although still forming a well-preserved microtexture with magnetite, have been oxidized subsolvus in ilmenite lamellae (Bjerkreim-Sokndal layered intrusion, sample 64-82 of Duchesne, 1972); (b) Dendritic lamellae of hercynitic spinel exsolutions in magnetite (Kagnuden deposit, sample 66-11 of Duchesne, 1999); (c) Cloth-textured magnetite in contact with an ilmenite grain. Note the absence of re-equilibration textures between ilmenite and magnetite (Bjerkreim-Sokndal layered intrusion, sample 66-216 of Duchesne, 1972). ilm ilmenite; $m t$ magnetite; $s p$ hercynitic spinel. 

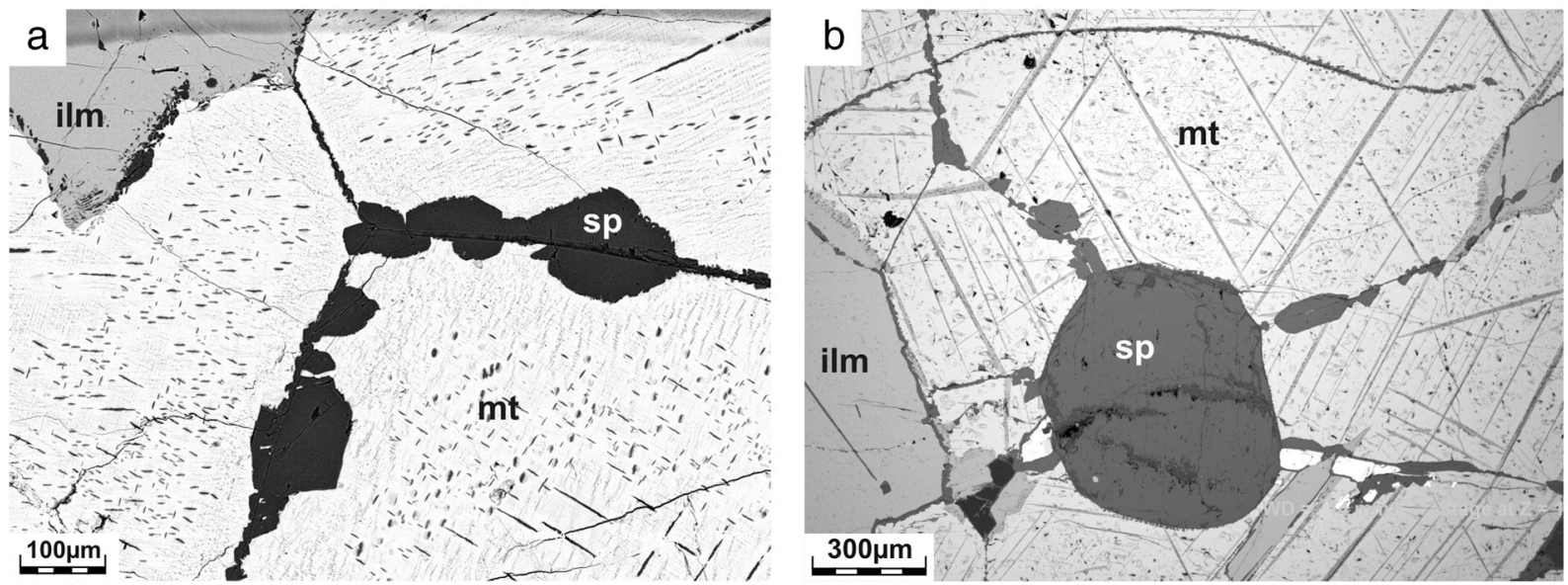

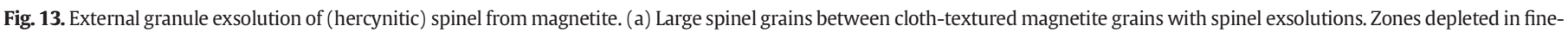

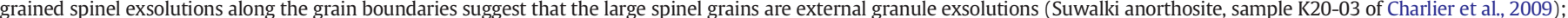

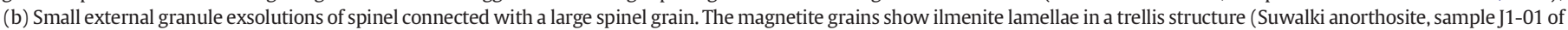
Charlier et al., 2009). ilm ilmenite; $m t$ magnetite; $s p$ hercynitic spinel.

$\mathrm{MgO}$ content of Fe-Ti oxides. Such re-equilibration has been described in the Kiglapait layered intrusion where Ti-magnetite has lost Mg to associated olivine and augite (Morse, 1980). In the Tellnes deposit, re-equilibration of ilmenite with the trapped liquid continued to lower temperatures by re-equilibration with ferromagnesian minerals, particularly orthopyroxene (Charlier et al., 2007). The MgO re-equilibration can be quantified using the QUILF algorithm (e.g. Frost and Lindsley, 1992; Lindsley and Frost, 1992).
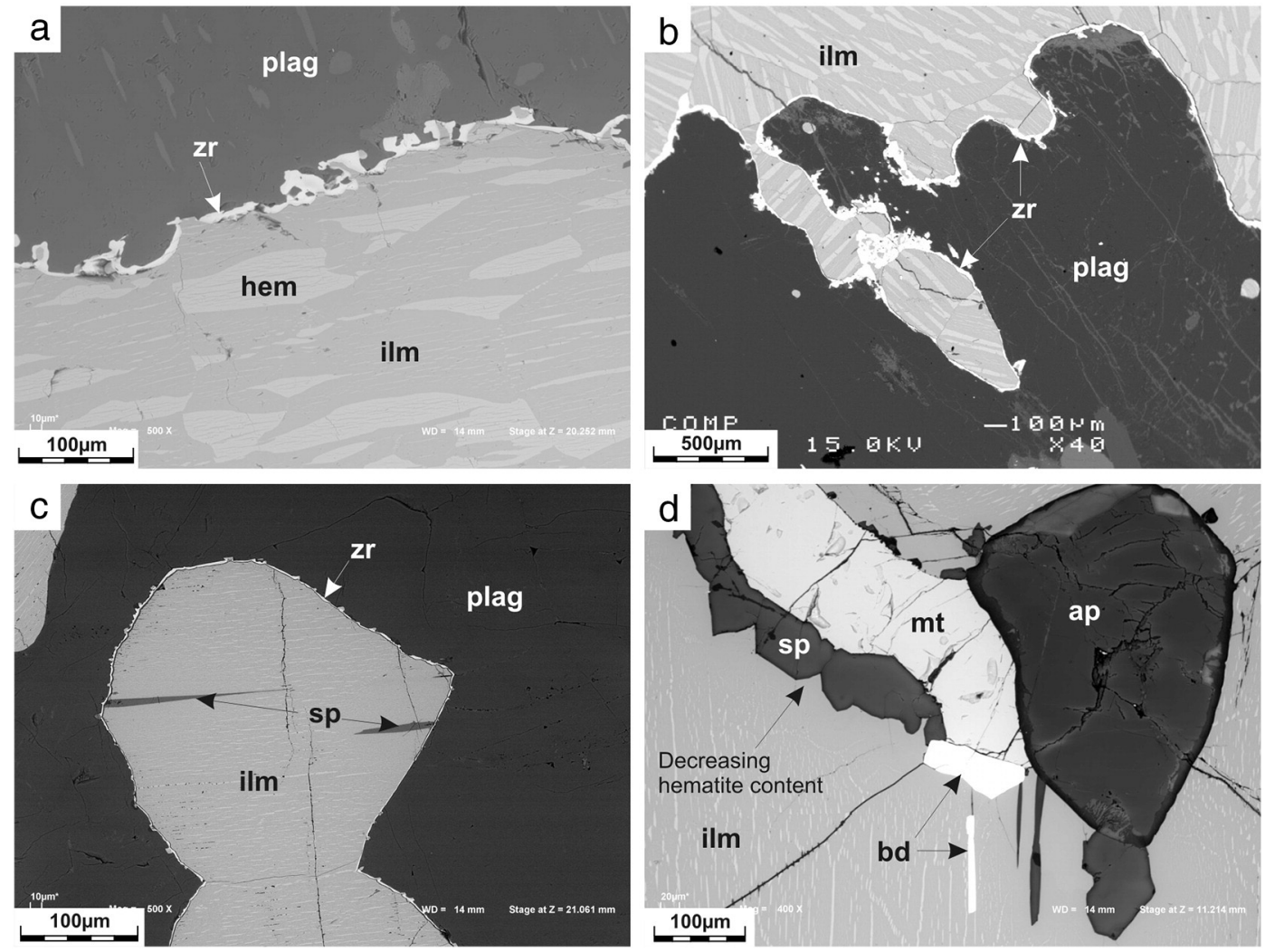

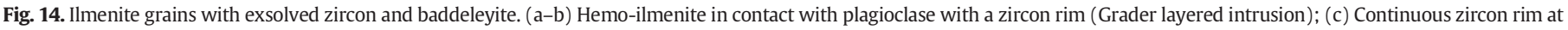

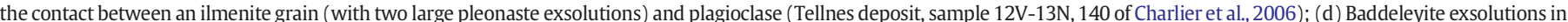

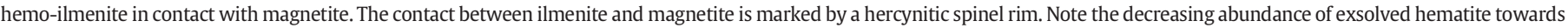

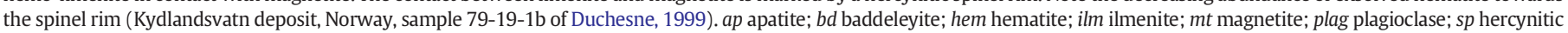
spinel; zr zircon. 

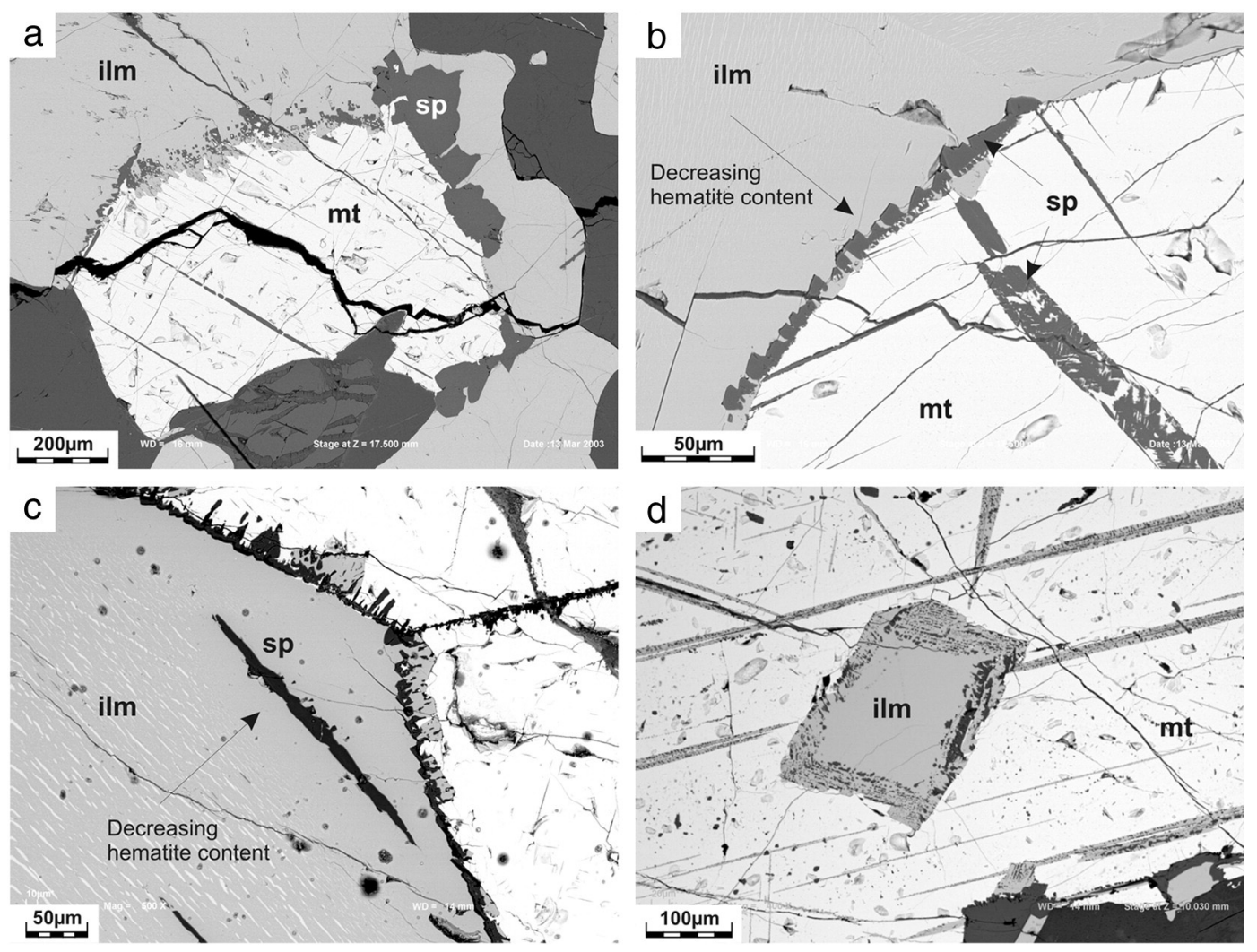

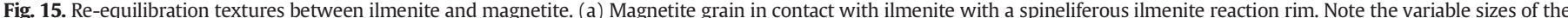

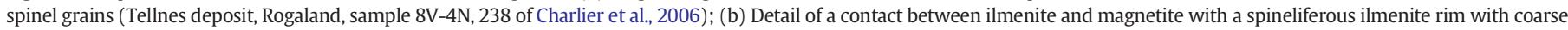

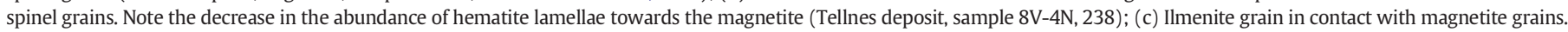

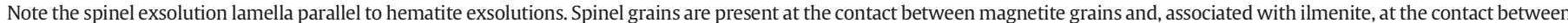

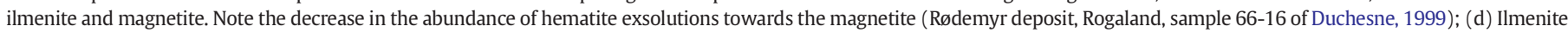

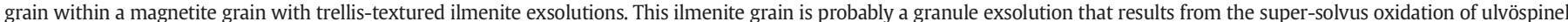

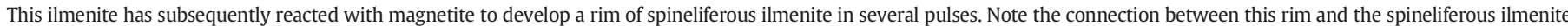
lamellae at the upper right corner of the ilmenite grain (Suwalki anorthosite, sample J1-01 of Charlier et al., 2009). ilm ilmenite; $m t$ magnetite; sp hercynitic spinel.

and hematite-ilmenite solid solutions (Carmichael, 1961). Ulvöspinel exsolutions in magnetite have been documented in various localities, e.g. the Skaergaard intrusion (Vincent and Phillips, 1954; Vincent, 1960), the Fongen-Hyllingen complex (Thy, 1982), and the Fedorivka layered intrusion (Duchesne et al., 2006). However, ilmenite exsolutions in magnetite are more common and result from the oxidation of the ulvöspinel

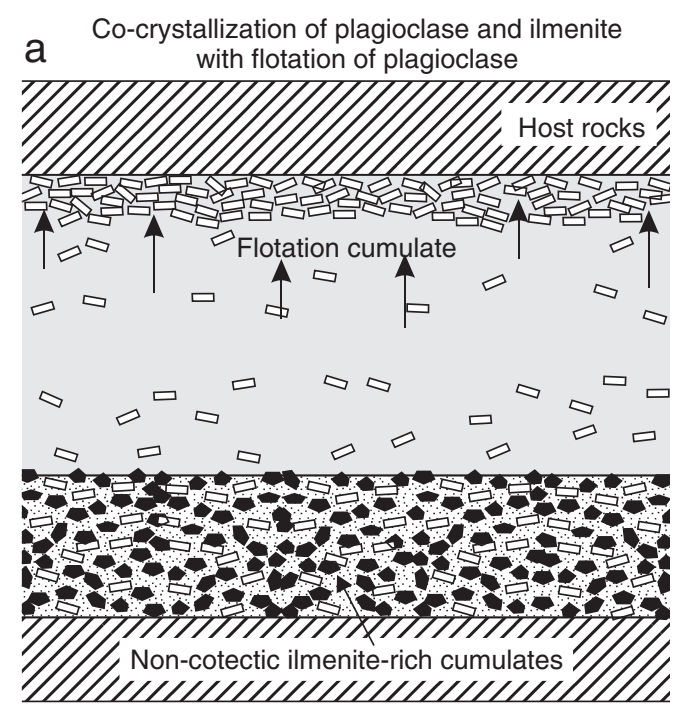

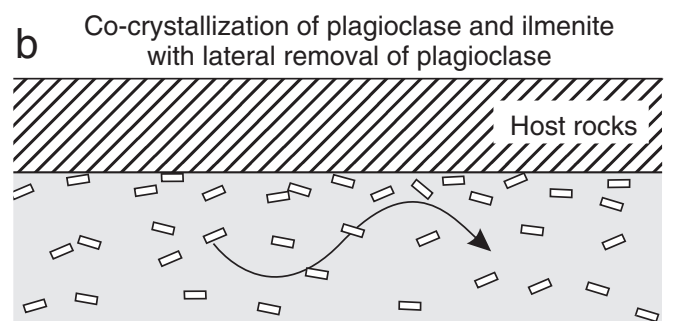

Lateral removal of plagioclase

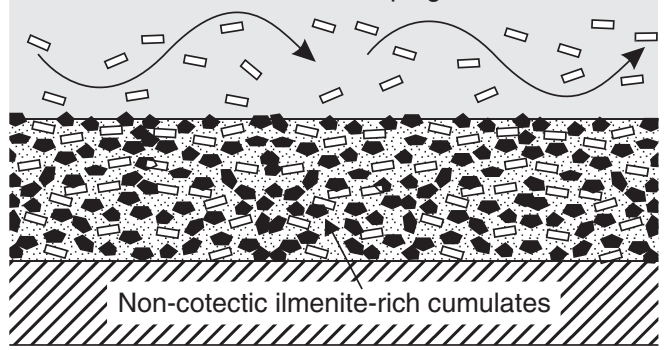

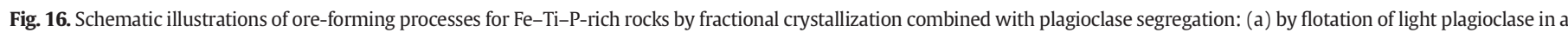
dense Fe-Ti-rich melt; (b) by lateral removal driven by convection. 

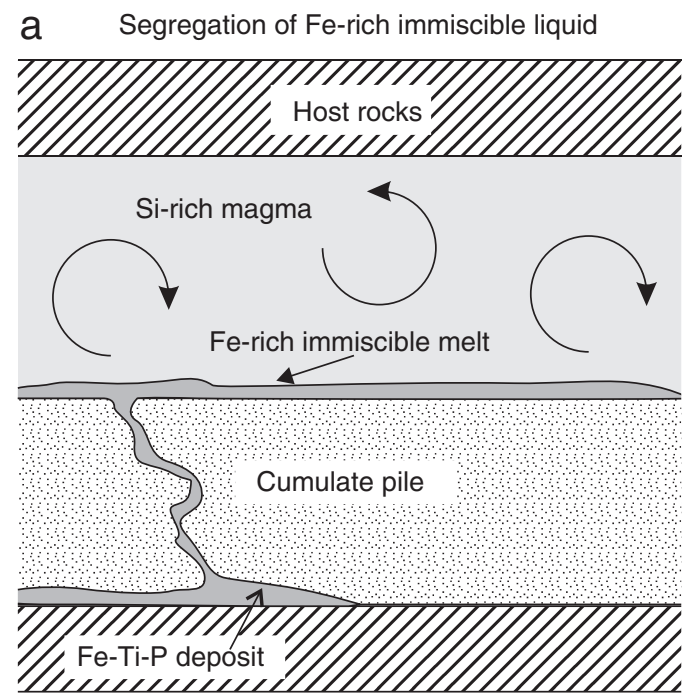

b Segregation of Fe-rich immiscible liquid and

b formation of Fe-Ti-P-ric cumulates

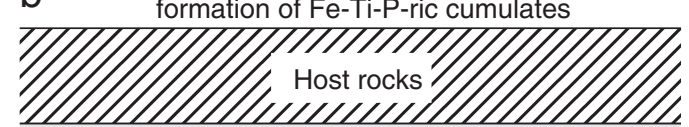

Si-rich magma

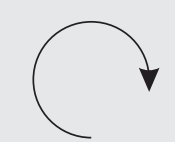

4

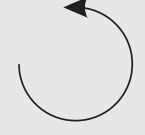

Cumulates from Fe-rich melt
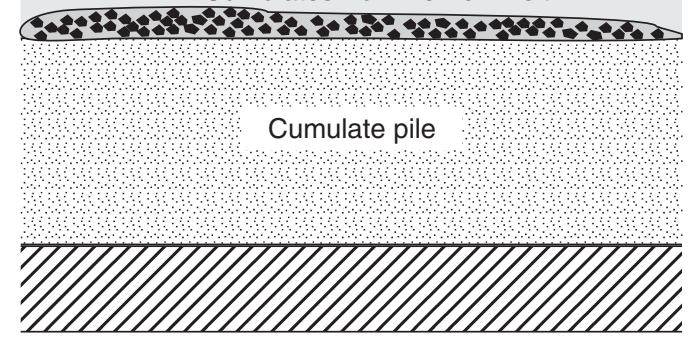

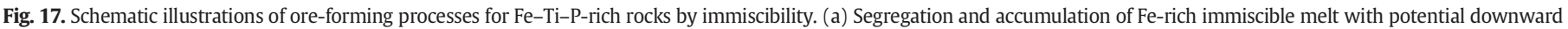
percolation of the dense melt into partially molten cumulate; (b) Segregation of an immiscible Fe-rich melt and crystallization of an Fe-Ti oxide-rich cumulate.

component in the reaction: $3 \mathrm{Fe}_{2} \mathrm{TiO}_{4}+1 / 2 \mathrm{O}_{2} \rightarrow 3 \mathrm{FeTiO}_{3}+\mathrm{Fe}_{3} \mathrm{O}_{4}$. This reaction usually takes place above the magnetite-ulvöspinel solvus to produce a trellis of ilmenite lamellae in magnetite. It is commonly observed that this reaction can also affect cloth intergrowths of ulvöspinel lamellae in magnetite by subsolvus transformation of ulvöspinel into microlamellae of ilmenite without notably affecting the cloth microtexture (Duchesne, 1970).

Ilmenite may exsolve lenses of hematite when the hematite content exceeds 7-9 wt.\%. It is then referred to as hemo-ilmenite (Basley and Buddington, 1958). Two generations of hematite exsolutions may develop above $18 \mathrm{~mol} \%$ of hematite. Exsolutions of aluminous spinel (hercynitic spinel, $(\mathrm{Mg}, \mathrm{Fe}) \mathrm{Al}_{2} \mathrm{O}_{4}$ ) commonly occur in Ti-magnetite (Fig. 13) and, more rarely, in ilmenite (Fig. 14c). Small grains are also observed in treillis lamellae of spineliferous ilmenite in magnetite, in reaction rims at the contact between primary ilmenite and magnetite,

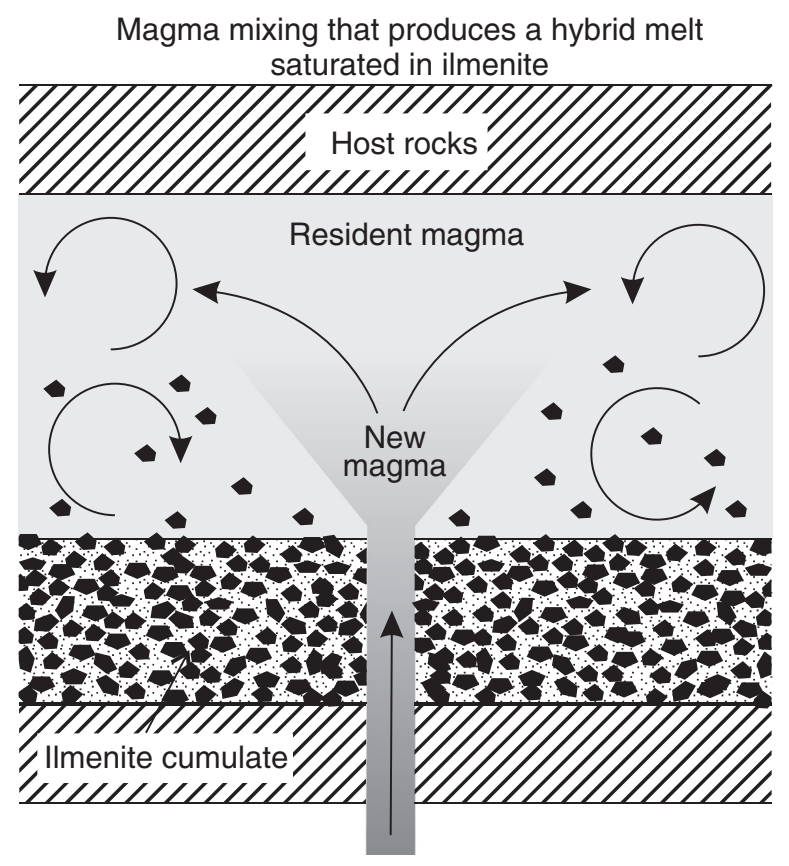

Fig. 18. Schematic illustration of ore-forming process for ilmenite-rich rocks by magma mixing and production of a hybrid magma located in the stability field of ilmenite. and in external granules. This reflects high primary $\mathrm{Al}$ and $\mathrm{Mg}$ contents of the host magnetite, and the low solubility of $\mathrm{Al}_{2} \mathrm{O}_{3}$ in ilmenite (Spencer and Lindsley, 1981). Rare lamellae of magnetite parallel to hematite exsolutions occur in hemo-ilmenite grains of the Lac Tio ore (Robinson et al., 2013; Bolle et al., 2014). They result from a hightemperature, localized reduction-exsolution process that preceded the normal lower-temperature exsolutions of hematite (Robinson et al., 2013).

Naslund (1987) has described baddeleyite lamellae in ilmenite from the tholeiitic Basistoppen sill. This texture is interpreted as resulting from subsolidus exsolution of $\mathrm{Zr}$ from the ilmenite lattice. Similar textures with zircon, baddeleyite and srilankite $\left(\mathrm{Ti}_{2} \mathrm{ZrO}_{6}\right)$ associated with ilmenite have also been described by Bingen et al. (2001) in high-grade meta-anorthositic rocks from Western Norway. They interpret the occurrence of baddeleyite as external granule exsolutions from ilmenite during subsolidus cooling of the magmatic intrusion. Zircon would have been produced by the Sveconorwegian granulitefacies metamorphism due to the reaction of baddeleyite with available silica. The random position of zircon coronas relative to hematite exsolutions in ilmenite suggests that $\mathrm{Zr}$ exsolution occurred before hematite exsolution (Bingen et al., 2001). This is also a common feature in Fe-Ti deposits (Fig. 14; Charlier et al., 2007; Morisset and Scoates, 2008).

\subsection{Reaction between ilmenite and magnetite}

Upon cooling, coexisting Ti-magnetite and ilmenite undergo $\mathrm{Fe}^{2+} \mathrm{Ti}^{4+}$ for $2 \mathrm{Fe}^{3+}$ exchange, with the magnetite gaining $\mathrm{Fe}^{3+}$ and the ilmenite gaining $\mathrm{Fe}^{2+} \mathrm{Ti}^{4+}$, leading to natural "purification" of both minerals (Fig. 15; Buddington and Lindsley, 1964; Duchesne, 1972). Of course, no reaction rim of aluminous spinel is observed when primary ulvöspinel exsolutions in magnetite are preserved (Fig. 12c), though it is possible that some ulvöspinel contents may have reacted to ilmenite above the ulvöspinel-magnetite solvus. This does not exclude that such magnetites do show exsolution of aluminous spinel in the cubic planes, probably formed earlier than the ulvöspinel lamellae. The relative proportions of magnetite and ilmenite are crucial for determining the degree of re-equilibration between these two phases (Frost et al., 1988). If the proportion of magnetite compared to ilmenite is very small, this reaction may completely convert the primary magnetite into Ti-poor magnetite without significantly affecting the composition of ilmenite. 


\section{Ore-forming processes}

\subsection{Fractional crystallization, crystal sorting and plagioclase buoyancy}

Magmas parental to anorthosite, and their residual liquids, are Ti-rich, commonly having more than 4 wt.\% $\mathrm{TiO}_{2}$ (e.g. Mitchell et al., 1996; Vander Auwera et al., 1998). This may promote early ilmenite saturation, as shown by Toplis and Carroll (1995), who have suggested that crystallization of ilmenite as the first oxide is controlled by the $\mathrm{TiO}_{2}$ content of the melt. In the Tellnes deposit (Charlier et al., 2006), the Grader layered intrusion (Charlier et al., 2008) and the Lac Tio mine (Charlier et al., 2010b), plagioclase and ilmenite are the first liquidus phases. In more differentiated cumulates, ilmenite and plagioclase may be accompanied by olivine, orthopyroxene, clinopyroxene, magnetite and apatite. Calculations of the cotectic proportions of ilmenite in ferrobasaltic liquids yield values of ca. 20 wt.\% (Charlier et al., 2007, 2008). This proportion of ilmenite in a cumulate corresponds to ca. $8 \mathrm{wt} . \% \mathrm{TiO}_{2}$ in the rock, which is not sufficient to form a titanium ore. Enrichment processes for ilmenite are therefore necessary.

Melts associated with anorthosite also have high concentrations of $\mathrm{FeO}_{\text {tot }}$, which exert a strong influence on the density of the melt. Several studies have discussed the "plagioclase-magma density paradox" (Morse, 1973; Campbell et al., 1978; Scoates, 2000), i.e. that intermediatecomposition plagioclase $\left(\mathrm{An}_{40-60}\right)$ is less dense than the melt with which it is in equilibrium. This is responsible for the buoyant accumulation of plagioclase at the top of a deep-seated magma chamber in the classical model of Emslie (1980). It also plays an important role in the crystallization of rocks that are associated with anorthosite at the final emplacement depth (Vander Auwera et al., 2006). In melts saturated with plagioclase $+\mathrm{Fe}-\mathrm{Ti}$ oxides, the different densities of these minerals can result in flotation of plagioclase but sinking of the dense Fe-Ti oxides $\left(\rho_{\text {ilmenite }}=4.7, \rho_{\text {magnetite }}=5.2\right.$ ). This cumulate sorting produces Fe-Ti oxide abundance greater than the cotectic proportions would suggest (Charlier et al., 2007, 2009). Plagioclase can float vertically and form anorthosite that is hard to distinguish from the host anorthosite (Fig. 16a), or be transported laterally to form leucocratic rocks in other parts of the magma chamber, such as in the Grader layered intrusion (Fig. 16b; Charlier et al., 2008).

\subsection{Immiscibility}

Immiscibility between two silicate melts (ferrobasalt-rhyolite) has been recognized experimentally and in natural systems as a potential differentiation mechanism in evolved basaltic magmas (Roedder, 1978; Philpotts, 1982; Charlier and Grove, 2012; Charlier et al., 2013). Philpotts (1967) proposed that immiscibility is responsible for the origin of certain Fe-Ti oxide and apatite rocks. Kolker (1982) also favored this model for the origin of nelsonites, mainly to explain field evidence such as discordant veins and dykes intruding the host anorthosite (Fig. 17a). A similar process was invoked by Force (1991) to generate ilmenite deposits in general. However, data presented by Philpotts (1967) for the immiscibility of an Fe-Ti-P-rich melt were obtained at the unrealistically high temperature of $1420{ }^{\circ} \mathrm{C}$. Lindsley (2003) explains that he has never been able to generate Fe-Ti oxide liquids experimentally. He concluded from experimental evidence that oxide bodies crystallize from Fe-Ti-P-rich silicate melts and are emplaced either as a crystal mush or in the solid state. Moreover, the relative proportion of oxides and apatite, ca. $2 / 3$ oxides and 1/3 apatite (Kolker, 1982), does not necessarily indicate an origin related to immiscibility. This could instead simply reflect cotectic proportions of Fe-Ti oxides and apatite crystallizing from a silicate melt, which are not then sorted during the concentration process.

Duchesne (1999) and Dymek and Owens (2001) proposed instead that nelsonites represent cumulates. The absence or low abundance of ferromagnesian silicates could result from earlier saturation of ilmenite and apatite compared to ferromagnesian silicates. Liquidus phases would thus be plagioclase, ilmenite, \pm magnetite, and apatite. This unusual sequence of crystallization, with apatite crystallizing before ferromagnesian silicates, occurs in the Grader layered intrusion (Charlier et al., 2008). Liquidus plagioclase could also be removed by crystal sorting or flotation.

Another hypothesis that has not been fully considered is that nelsonites represent cumulates crystallized from iron-rich immiscible melts, rather than crystallized immiscible melts (Fig. 17b; Namur et al., 2012). In this scenario, the Fe-rich melt segregates from the Sirich melt. However, the ore-rock does not correspond to the immiscible liquid. Instead, the ore is a cumulate crystallized from the Fe-rich melt. The Si-rich melt will produce cumulate rocks with the same cumulus assemblage but different phase proportions (much more leucocratic).

Immiscibility should therefore be carefully considered as an important process in the formation of some Fe-Ti-P deposits. Liquid immiscibility is known to occur in the late evolution of tholeiitic basalt, and can potentially yield an Fe-Ti-P-rich melt that can produce ilmenite-magnetite-apatite-rich rocks associated with evolved plagioclase and low Mg\# ferromagnesian silicates (olivine, clinopyroxene). However, while immiscibility hypotheses for the origin of nelsonites and some evolved ferrogabbros deserve discussion and possibly further experiments, pure ilmenite ore, such as that in Jerneld (Duchesne, 1999) and Lac Tio (Lister, 1966), cannot be produced by immiscibility. Immiscibility in evolved ferrobasalts does not produce melts saturated with a single-phase: a liquid of ilmenite composition would melt at $1360^{\circ}$, an unrealistically high temperature for crustal conditions.

\subsection{Magma mixing}

The $\mathrm{Cr}$ concentration in ilmenite from the Lac Tio deposit displays normal and reverse fractionation trends upsection. Charlier et al. (2010b) interpreted these compositional characteristics as evidence for multiple pulses of magma emplacement and alternating periods of fractional crystallization and magma mixing. By analogy with the classical magma mixing hypothesis for the origin of some chromite deposits (Irvine, 1977), mixing of magmas would have produced hybrid magmas located in the stability field of ilmenite, resulting in crystallization of ilmenite alone after each mixing event. It is highly probable that the natural plagioclase-ilmenite cotectic is curved and that mixing of two magmas lying on the cotectic can produce a hybrid located in the stability field of ilmenite, which will therefore crystallize only ilmenite until the liquid joins the cotectic. This ilmenite would accumulate due to a high density contrast with the liquid. However, the paucity of rocks with cotectic proportions of ilmenite and plagioclase (1525 wt.\% ilmenite and 85-75 wt.\% plagioclase) leads us to suggest that the liquid did not follow the cotectic during major periods of fractionation (Fig. 18).

Robinson et al. (2003) suggested that magma mixing was responsible for the high proportion of ilmenite in Tellnes. However, in this deposit, ilmenite does not occur as the sole liquidus mineral and is usually associated at least with plagioclase. Additionally, the very narrow range of $\mathrm{Sr}$ isotope values in the Tellnes deposit (Charlier et al., 2006) does not favor such mixing as a mechanism for ilmenite enrichment in Tellnes.

\subsection{Filter-press compaction}

Anorthosite plutons are emplaced as diapirs and crystallize during the ascent of the plagioclase mush. This dynamic emplacement provides the conditions necessary to produce stress-driven melt segregation in partially molten rocks (Kohlstedt et al., 2010). The result is the formation of pure anorthosite (commonly $>95 \%$ plagioclase) that is segregated from the residual melt (enriched in Fe-Ti-P). The residual melt can be collected in the margins of massif anorthosites, where mafic intrusions are commonly observed (Fig. 19; Vander Auwera et al., 2006). 


\subsection{Solid-state remobilization}

It is not rare to observe veins or seams of pure hemo-ilmenite in massif-type anorthosites. Their emplacement as a melt is highly questionable because the melting point of hemo-ilmenite (at least $1365{ }^{\circ} \mathrm{C}$, depending on the amount of dissolved hematite in the solid solution) is hotter than typical magmatic temperature. In Fe-Ti-rich cumulate rocks, ilmenite and magnetite are always interstitial to the silicate minerals, even when they crystallize on the liquidus after plagioclase and before pyroxenes, as observed in the Bjerkreim-Sokndal layered intrusion (Wilson et al., 1996) and in experiments (Vander Auwera and Longhi, 1994). In these rocks, ilmenite tends to concentrate in low-stress zones such as pressure shadows (Paludan et al., 1994; Duchesne, 1996). It is thus observed that ilmenite can easily recrystallize and migrate by diffusion creep in subsolidus conditions. It can be inferred that such a migration process also occurs on a larger scale during high-temperature deformation linked to anorthosite emplacement, giving rise to massive veins of ilmenite (Duchesne, 1996, 1999).

\subsection{Hydrothermal remobilization}

Plagioclase in anorthosite massifs is commonly altered to various degrees and significant volumes of anorthosite can be affected. The alteration produces white-colored altered anorthosite, as opposed to dark-colored fresh anorthosite. This process has been observed in the Damiao anorthosite by Li et al. (2014), who show that alteration of plagioclase reduces the Fe and $\mathrm{Ti}$ contents of the anorthosite. They thus interpret the formation of high-grade Fe-Ti-P ores as a hydrothermal process involving $\mathrm{P}$ - and $\mathrm{F}$-rich fluids that migrate, alter the anorthosite and transport Fe and Ti. These elements would precipitate in other parts of the anorthosite and form veined or lenticular massive ore bodies (Fig. 20). This interpretation is further supported by low homogenization temperatures of fluid inclusions in apatite, ranging from $180{ }^{\circ} \mathrm{C}$ to $420{ }^{\circ} \mathrm{C}$ ( Li et al., 2010). Simple mass balance calculations taking into account the compositions of the altered and fresh anorthosite make this hypothesis reasonable. However, more insights are needed into the mobility of titanium and other immobile elements by fluids, although Ti has been shown to be mobile in chloride and fluoride-bearing fluids (Rapp et al., 2010).

Segregation of Fe-Ti-rich interstitial melt from uprising anorthosite mush

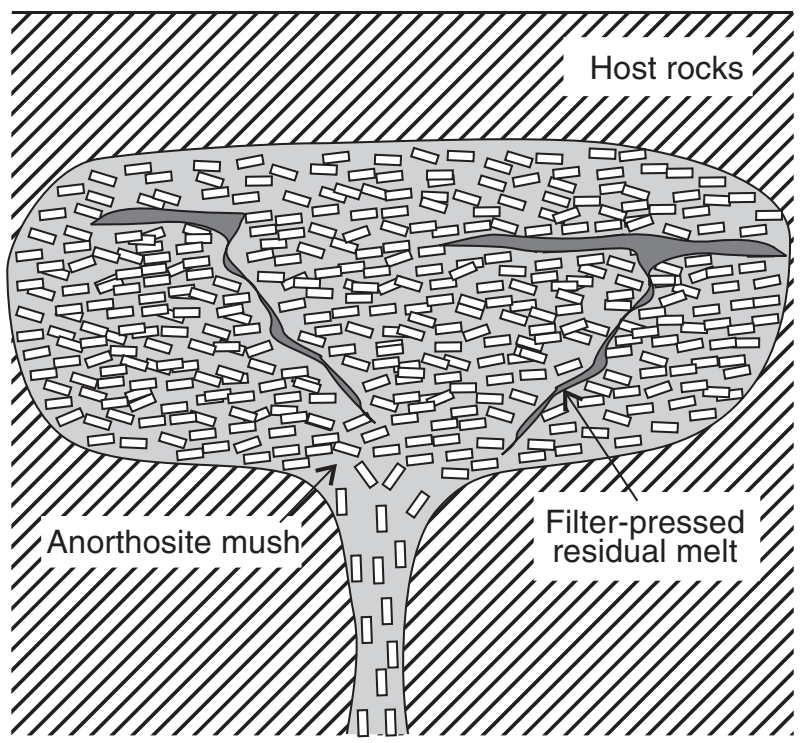

Fig. 19. Schematic illustration of ore-forming process for Fe-Ti-P-rich rocks by segregation of Fe-Ti-enriched residual melts from uprising anorthosite mush.
Hydrothermal remobilization of $\mathrm{Fe}$ and $\mathrm{Ti}$ from plagioclase

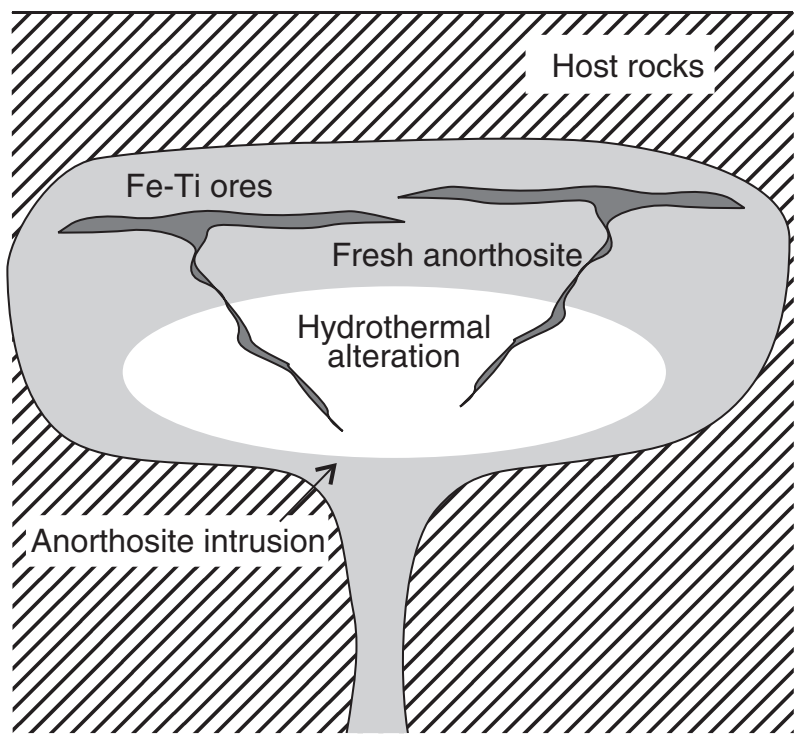

Fig. 20. Schematic illustration of ore-forming process for Fe-Ti-P-rich rocks by hydrothermal remobilization of Fe and Ti followed by fluid concentration in veins and other discordant bodies.

\section{Metamorphism: a natural beneficiation processes?}

Fe-Ti oxide minerals undergo substantial chemical modification through the various processes described in the previous sections. In general, during subsolidus cooling, magnetite tends to lose $\mathrm{Ti}, \mathrm{Al}$ and $\mathrm{Mg}$ through external granule exsolution of spinel-bearing ilmenite and primary ilmenite progressively reduces its hematite and hercynite contents. Both oxides thereby approach their pure end-member compositions and purge some of their minor elements. To what extent these subsolidus modifications can increase the industrial value of an ore is an interesting question to examine because it can influence exploration strategy. One immediately thinks of deposits that have been metamorphosed at greenschist-facies conditions and thus spent a lot of time in the appropriate temperature range. Moreover, deformation could also have favored strain-induced exsolution and recrystallization of the minerals, as seen in the Rooiwater ore body (Reynolds, 1986). However, it must be stressed on theoretical grounds that this natural purification process has its limits. The final major element contents of both oxides will be largely controlled by the $\mathrm{T}-\mathrm{fO}_{2}$ conditions during re-equilibration. The ilmenite $\mathrm{Mg}$ content will depend on complex interactions with the accompanying silicates minerals, if any. The trace elements will be redistributed between ilmenite, magnetite and spinel. The bulk $\mathrm{V}$ and $\mathrm{Cr}$ contents of the ore body will not change, but $\mathrm{V}$ and $\mathrm{Cr}$ contents in ilmenite will decrease with the ferric iron content; these elements will probably then become concentrated in magnetite or a spinel phase. These phenomena require further investigation.

\section{Magnetic signatures of Fe-Ti-V-P deposits}

Aeromagnetic surveying is an essential geophysical tool for the exploration of Fe-Ti-V-P ore bodies, as exemplified by the discoveries of the world-class Lac Tio and Tellnes deposits, both of which were found during aeromagnetic measurements campaigns (Bourret, 1949; Gierth and Krause, 1973). The interpretation of such surveys strongly depends on the magnetic properties of the rocks being explored, especially the ore deposits themselves. Characterization of rock magnetic properties in the Rogaland Anorthosite Province led McEnroe et al. (2001) to distinguish between two groups of Fe-Ti mineralization 
types that produce large and contrasting anomalies on aeromagnetic maps, a classification that can be extended to Fe-Ti oxide deposits worldwide.

The first group of Fe-Ti mineralizations encompasses noritic rocks with relatively abundant coarse (multi-domain) magnetite and homogeneous (near-end-member) ilmenite. Ores from this group have high values of natural remanent magnetization (NRM) and magnetic susceptibility $(K)$, coupled with low values of coercivity and Koenigsberger ratios $(Q$, the ratio of NRM to induced magnetization i.e. $K$ multiplied by the ambient magnetic field). They produce an induced-current magnetic response parallel to the Earth's present-day magnetic field, giving rise to positive anomalies on aeromagnetic maps. The magnetic properties of these rocks are dominated by magnetite; in particular, the viscous NRM behavior is "more or less as predicted from the common behavior of multi-domain magnetite" (McEnroe et al., 2001). Evolved cumulates from the upper part of the layered series of the Bjerkreim-Sokndal intrusion are typical examples of magnetiterich noritic rocks with homogeneous ilmenite giving rise to induced magnetic highs. Mean NRM, $K$ and $Q$ values for these layered units are in the range of $2-10 \mathrm{~A} / \mathrm{m}, 90-280 \times 10^{-3}$ SI and $0.5-1$, respectively (calculated from McEnroe et al., 1996, 2009).

The second group of Fe-Ti ore deposits, with a magnetic signature drastically different from the former group, includes hemo-ilmeniterich noritic rocks and massive hemo-ilmenite ores, containing no or minor multi-domain magnetite. Rocks from this group have high NRM and Q values, and moderate to high coercivities and susceptibilities. They produce remanence-influenced to remanence-dominated anomalies, and are thus strongly dependent on the orientation of the Earth's magnetic field at the time of emplacement and cooling. The strong and stable NRM of this group results primarily from hemo-ilmenite; however, oxide exsolutions in silicates, chiefly exsolved blades and rods of hemo-ilmenite and/or magnetite with ilmenite oxy-exsolution in pyroxenes may contribute significantly to NRM in some cases (McEnroe et al., 2001). The contributions of hemoilmenite and pyroxene with oxide exsolutions may both be explained by a peculiar ferrimagnetic substructure created in the exsolution intergrowths (McEnroe et al., 2002, 2004, 2007), following the theory of lamellar magnetism (e.g. Robinson et al., 2002). Most other magnetic properties of hemo-ilmenite ores are also strongly influenced by lamellar magnetism, with the notable exception of magnetic susceptibility: at the grain-scale, the exsolution lamellae and their host contribute to the intrinsic magnetic susceptibility according to their respective proportions and susceptibilities.

The Tellnes and Lac Tio ore bodies are typical examples of Fe-Ti deposits with NRM dominated by hemo-ilmenite. Both ore bodies produce negative anomalies (magnetic lows) related to NRM acquired during a time of reversed magnetic polarity (McEnroe et al., 2001, 2007). The anomaly over the Tellnes open-pit is hardly distinguishable from the negative anomaly generated by the surrounding Åna-Sira anorthosite. It is worth noting that not all anorthosite bodies generate negative anomalies; anorthosites have quite variable magnetic properties and therefore, aeromagnetic signatures, depending on their oxide mineralogy (Brown et al., 2011). The Tellnes and Lac Tio deposits have very different average NRM and $Q$ values $(7 \mathrm{~A} / \mathrm{m}$ and 7.5 , and $47 \mathrm{~A} / \mathrm{m}$ and 100 , respectively), with similar average $K$ values $\left(29 \times 10^{-3} \mathrm{SI}\right.$ and $32 \times 10^{-3} \mathrm{SI}$, respectively; calculated from Hargraves, 1959; Diot et al., 2003; McEnroe et al., 1996, 2007; Bolle et al., 2014). The $K$ values in both deposits are strongly influenced by the proportions of magnetite, when present either as discrete primary grains, exsolution lamellae in pyroxenes or hemo-ilmenite, or secondary minerals, since this oxide has a very strong intrinsic magnetic susceptibility. For example, in the Lac Tio deposit, massive hemo-ilmenite samples with no or negligible magnetite have $K$ values lower than $7-8 \times 10^{-3}$ SI, whereas ilmenitites and hemo-ilmenite-rich noritic rocks containing minor magnetite have susceptibilities up to $113 \times 10^{-3}$ SI (data from Hargraves, 1959; McEnroe et al., 2007; Bolle et al., 2014).
The magnetic signature may vary across a single deposit, as shown by the Storgangen ore body. The magnetic anomaly over this deposit grades westward from a negative remanence-dominated anomaly to a positive induced anomaly, most likely related to an increase in the magnetite/hemo-ilmenite ratio from east to west (McEnroe et al., 2001). There are also Fe-Ti mineralizations that depart somewhat from the classification of McEnroe et al. (2001). For example, a cumulate unit from the upper part of the layered series of the Bjerkreim-Sokndal intrusion has high NRM, $K$ and $Q$ values (average of $19 \mathrm{~A} / \mathrm{m}, 112 \times 10^{-3}$ SI and 5.8, respectively; calculated from McEnroe et al., 2009) and produces a striking negative remanent magnetic anomaly, known as the Heskestad anomaly, reflecting the coexistence of, and competition between, multi-domain magnetite and hemo-ilmenite + oxide exsolutions in pyroxenes (McEnroe et al., 2004, 2009). A synthesis of the different types of Fe-Ti mineralization according to their magnetic properties is presented in Table 3.

\section{Structural analysis and deformation}

The most conspicuous structural elements observed at the outcrop scale in many $\mathrm{Fe}-\mathrm{Ti}$ ore bodies are igneous layering and/or mineral lamination (mostly defined by plagioclase and orthopyroxene crystals). Fe-Ti oxides, especially hemo-ilmenite, commonly display a shapepreferred orientation, with a flattening plane usually parallel to the mineral lamination. This can be coupled with a lattice-preferred orientation of the grains, as demonstrated using electron backscattered diffraction on samples from the Lac Tio deposit and a nearby minor hemoilmenite deposit (Robinson et al., 2013; Bolle et al., 2014). At the microscale, evidence of intracrystalline deformation (undulose extinction, subgrains, bending, kinking) is common in silicates (mostly plagioclase and orthopyroxene) from all types of Fe-Ti deposits, as is partial dynamic recrystallization into small grains (Paludan et al., 1994; Duchesne, 1999; Diot et al., 2003; Bolle et al., 2014). Ilmenite shows virtually no evidence of intracrystalline deformation, which reflects its aptitude for recrystallizing, as discussed above. This recrystallization does not only occur only during deformation, but commonly continues after deformation has ceased (static recrystallization), as demonstrated by the texture of massive ores: aggregated hemoilmenite grains have polygonal shapes with boundaries tending to make triple junctions with dihedral angles of $\mathrm{ca} .120^{\circ}$, and they have been coarsened, as suggested by the smaller size of ilmenite grains included in plagioclase. Such a texture is typical of grain boundary area reduction (also called textural coarsening in igneous rocks; Higgins, 1998), a process of grain boundary migration resulting in grain growth and straightening of grain boundaries, which is the principal mechanism of static recrystallization (Passchier and Trouw, 2005).

The magnetic fabrics measured in the Tellnes and Lac Tio deposits (Diot et al., 2003; Bolle et al., 2014) are dominated by shape-preferred orientation of magnetite and/or lattice-preferred orientation of hemoilmenite. The magnetic foliation, which proxies for igneous layering and mineral lamination, displays patterns that (1) mimic the 3D shape of the two deposits (namely an elongated trough for the Tellnes ore body and a funnel for the Lac Tio deposit) and (2) express their cylindrical folding around a gently-plunging axis. The magnetic lineation reveals in both deposits a linear arrangement of the rock-forming minerals, most probably a mineral lineation that approximates the axis of maximum finite stretching. On average, it is parallel in both deposits to the gently-plunging fold axis revealed by the layout of the magnetic foliation. Bolle et al. (2014) also showed that the basinshaped Grader layered intrusion, which crops out only ca. $4 \mathrm{~km} \mathrm{SW}$ of the Lac Tio deposit, is folded around an axis with an orientation similar to the stretching direction suggested by the AMS analysis of the Lac Tio deposit. The Bjerkreim-Sokndal layered intrusion in the Rogaland anorthosite province is also folded into a complex synformal syncline, 
Table 3

Types of Fe-Ti mineralization according to magnetic properties.

\begin{tabular}{|c|c|c|c|c|c|}
\hline \multicolumn{2}{|l|}{ Type $^{\mathrm{a}, \mathrm{b}}$} & \multicolumn{4}{|l|}{ Example $^{\mathrm{c}}$} \\
\hline \multirow[t]{2}{*}{ Magnetic mineralogy } & \multirow[t]{2}{*}{ Magnetic properties } & \multirow[t]{2}{*}{ Mineralization } & \multirow{2}{*}{$\frac{\mathrm{NRM}}{(\mathrm{A} / \mathrm{m})}$} & \multirow{2}{*}{$\frac{\mathrm{K}}{\left(10^{-3} \mathrm{SI}\right)}$} & \multirow[t]{2}{*}{ Q } \\
\hline & & & & & \\
\hline \multirow[t]{3}{*}{ Mag + homogeneous Ilm } & \multirow{3}{*}{$\begin{array}{l}\text { High NRM and } K \text {, low coercivity and } Q \\
\text { (induced, positive magnetic anomaly) }\end{array}$} & Mydland (BKSK SE lobe) ${ }^{\mathrm{d}}$ & 5 & 181 & 0.7 \\
\hline & & Bakka (BKSK S lobe) ${ }^{\mathrm{d}}$ & 10.5 & 279 & 1 \\
\hline & & MCUIVf (BKSK N lobe) ${ }^{\mathrm{b}}$ & 2 & 90 & 0.5 \\
\hline \multirow{2}{*}{ Hem-Ilm \pm Mag \pm Ox in Px } & \multirow{2}{*}{$\begin{array}{l}\text { High NRM and } Q \text {, moderate to high coercivity and } K \\
\text { (remanence-influenced to remanence-dominated magnetic anomaly, } \\
\text { with sign dependent on the paleofield orientation) }\end{array}$} & Tellnes mine $\mathrm{d}^{\mathrm{d}-\mathrm{f}}$ & 7 & 29 & 7.5 \\
\hline & & Lac Tio deposit ${ }^{\mathrm{e}, \mathrm{g}-\mathrm{i}}$ & 47 & 32 & 100 \\
\hline $\begin{array}{l}\text { Mag }+ \text { Hem-Ilm }+ \text { Ox in Px } \\
\quad \text { (peculiar case) }\end{array}$ & $\begin{array}{l}\text { High NRM, } K \text { and } Q \\
\text { (remanence-dominated magnetic anomaly) }\end{array}$ & $\begin{array}{l}\text { MCUIVe', Heskestad } \\
\text { (BKSK N lobe) }^{\mathrm{b}, \mathrm{e}}\end{array}$ & 19 & 112 & 5.8 \\
\hline
\end{tabular}

Symbols for minerals: Hem-Ilm, hemo-ilmenite; Ilm, ilmenite; Mag, magnetite; Ox, oxide; Px, pyroxene.

Other: NRM, natural remanent magnetization; $K$, magnetic susceptibility; $Q$ Koenigsberger ratio; BKSK, Bjerkreim-Sokndal layered intrusion.

a McEnroe et al. (2001).

b McEnroe et al. (2009).

c NRM, $K$ and $Q$ values are averages calculated from raw data.

d McEnroe et al. (1996).

e The associated magnetic anomaly is negative.

f Diot et al. (2003) (only for $K$ ).

g Hargraves (1959) (data recalculated).

h McEnroe et al. (2007).

i Bolle et al. (2014) (only for K).

divided into three lobes intersecting in a central funnel-shaped trough (Paludan et al., 1994; Bolle et al., 2000, 2002).

From the ideas developed above, it can be concluded that a hightemperature, syn- to post-emplacement deformation overprinting the primary magmatic structure is very common in $\mathrm{Fe}-\mathrm{Ti}$ ore bodies associated with anorthosites. According to Duchesne (1999), macroto microstructural deformation features observed in many deposits enclosed in anorthosite bodies from the Rogaland anorthosite province (e.g. Storgangen) would have been induced by the diapiric emplacement of the anorthosite plutons (Barnichon et al., 1999). The morphology, microstructures and magnetic fabric of the Tellnes ilmenite deposit were interpreted by Diot et al. (2003) as resulting from the synemplacement deformation of a noritic crystal mush injected into a strike-slip weakness zone cutting across the Åna-Sira anorthosite. This interpretation has been challenged by Charlier et al. (2006), who proposed that the Tellnes ore body represents a sill deformed during gravity-induced subsidence due to the higher density of the Fe-Ti oxide-rich rocks compared to the less-dense host anorthosite, possibly coupled with up-doming of the latter. This deformation mechanism is similar to that proposed to explain the deformation of the BjerkreimSokndal layered intrusion (Paludan et al., 1994; Bolle et al., 2000, 2002). Syn-emplacement, gravitational subsidence has also been invoked to explain the boudinage of anorthosite layers and the occurrence of meter-scale isoclinal slump folds in the Lac Tio and Grader ore bodies (Bolle et al., 2014). However, the main deformation event in both deposits, producing their folding and recorded by the magnetic fabric in Lac Tio, would correspond to ballooning of the anorthosite, which probably occurred during the gravitational deformation of the ore bodies (Bolle et al., 2014).

\section{Conclusions}

Proterozoic massif-type anorthosites and associated rocks are common hosts for Fe-Ti-V-P-enriched rocks. Major deposits are dominated by hemo-ilmenite, but Ti-magnetite and more rarely rutile are other important Ti-bearing minerals. Accompanying phases are plagioclase, orthopyroxene, clinopyroxene, olivine, and aluminous spinel. Apatite is common in the more evolved rock types. A range of magmatic processes may be responsible for the formation of these deposits. Fractional crystallization coupled with early saturation of ilmenite and plagioclase flotation is an efficient mechanism that adequately explains compositional variations in many deposits. Immiscibility of an Fe-Ti-(P)-rich melt has commonly been invoked, but more careful examination of this process should be undertaken, taking into account the most recent work on phase equilibria. Additional processes such as magma mixing, compaction, solid-state and hydrothermal remobilization deserve consideration. The large diversity of Fe-Ti ores associated with massiftype anorthosites is in accordance with the variety of their geological environments and the controlling factors on Fe-Ti oxide stability, composition and microtexture. Variables are as follows: parental magma composition, oxygen fugacity, morphology of host magma chamber and its deformation conditions, pressure of crystallization, and postcumulus evolution.

The dominant, less polluting chloride process for the extraction of $\mathrm{Ti}$ metal and $\mathrm{TiO}_{2}$ from ilmenite and rutile requires low $\mathrm{MgO}$ and $\mathrm{Cr}$ contents of the feedstock minerals. Unfortunately these elements are relatively abundant in ilmenite from the Tellnes and Lac Tio deposits, particularly in the lower part of the Tellnes deposit. Oxide-apatite gabbronorites are now considered as interesting targets because the composition of ilmenite in these rocks is $\mathrm{Cr}$ - and $\mathrm{Mg}$-poorer and the Ti-resource may be combined with phosphorous-bearing apatite. Ores from low-degree metamorphosed rocks should also be considered as important targets.

\section{Acknowledgments}

Titania A/S and Rio Tinto Iron and Titanium are gratefully acknowledged for their financial support. This paper has benefited from constructive comments by Kerry Stanaway and Ben Mandler. Reviews by Don Lindsley and an anonymous referee helped us improve the manuscript. Editorial handling by Arturo Gómez-Tuena is acknowledged.

\section{References}

Amelin, Y.V., Heaman, L.M., Verchogliad, V.M., Skobelev, V.M., 1994. Geochronological constraints on the emplacement history of an anorthosite - rapakivi granite suite: $\mathrm{U}-\mathrm{Pb}$ zircon and baddeleyite study of the Korosten complex, Ukraine. Contrib. Mineral. Petrol. 116, 411-419.

Anderson, A.T., Morin, M., 1969. Two types of massif anorthosite and their implications regarding the thermal history of the crust. In: Isachsen, Y.W. (Ed.), Origin of Anorthosite and Related Rocks. NY State Mus Sci Serv Mem, pp. 57-69.

Ashwal, L.D., 1982. Mineralogy of mafic and Fe-Ti oxide-rich differentiates of the Marcy anorthosite massif, Adirondacks, NY. Am. Mineral. 67, 14-27.

Ashwal, L.D., 1993. Anorthosites. Springer, Heidelberg.

Ashwal, L.D., 2010. The temporality of anorthosites. Can. Mineral. 48, 711-728.

Ashwal, L., Twist, D., 1994. The Kunene complex, Angola/Namibia: a composite massiftype anorthosite complex. Geol. Mag. 131, 579-591. 
Ashwal, L.D., Hamilton, M.A., Morel, V.P.I., Rambeloson, R.A., 1998. Geology, petrology and isotope geochemistry of massif-type anorthosites from southwest Madagascar. Contrib. Mineral. Petrol. 133, 389-401.

Ball, S.H., 1907. Titaniferous iron ores of Iron Mountain, Wyoming. U.S. Geol. Surv. Bull. 315, 206-212.

Barnes, S.J., 1986. The effect of trapped liquid crystallization on cumulus mineral compositions in layered intrusions. Contrib. Mineral. Petrol. 93, 524-531.

Barnichon, J.-D., Havenith, H., Hoffer, B., Charlier, R., Jongmans, D., Duchesne, J.C., 1999. The deformation of the Egersund Ogna massif, South Norway: finite element modelling of diapirism. Tectonophysics 303, 109-130.

Basley, J.R., Buddington, A.F., 1958. Iron-titanium oxide minerals, rocks and aeromagnetic anomalies of the Adirondacks area, N.Y. Econ. Geol. 53, 777-805.

Berg, J.H., 1977. Dry granulite mineral assemblages in the contact aureoles of the Nain complex, Labrador. Contrib. Mineral. Petrol. 64, 33-52.

Bhattacharya, A., Raith, M., Hoernes, S., Banerjee, D., 1998. Geochemical evolution of the massif-type anorthosite complex at Bolangir in the Eastern Ghats Belt of India. J. Petrol. 39, 1169-1195.

Bingen, B., Austrheim, H., Whitehouse, M., 2001. Ilmenite as a source for zirconium during high-grade metamorphism? Textural evidence from the Caledonides of Western Norway and implications for zircon geochronology. J. Petrol. 42, 355-375.

Bogdanova, S.V., Pashkevich, I.K., Buryanov, V.B., Makarenko, I.B., Orlyuk, M.I., Skobelev, V.M., Starostenko, V.I., Legostaeva, O.V., 2004. The 1.80-1.74-Ga gabbro-anorthositerapakivi Korosten Pluton in the Ukrainian Shield: a 3-D geophysical reconstruction of deep structure. Tectonophysics 381, 5-27.

Bolle, O., Diot, H., Duchesne, J.C., 2000. Magnetic fabric and deformation in charnockitic igneous rocks of the Bjerkreim-Sokndal layered intrusion (Rogaland, Southwest Norway). J. Struct. Geol. 22, 647-667.

Bolle, O., Trindade, R.I.F., Bouchez, J.L., Duchesne, J.C., 2002. Imaging downward granitic magma transport in the Rogaland Igneous Complex, SW Norway. Terra Nova 14, 87-92.

Bolle, O., Demaiffe, D., Duchesne, J.C., 2003. Petrogenesis of jotunitic and acidic members of an AMC suite (Rogaland anorthosite province, SW Norway): a Sr and Nd isotopic assessment. Precambrian Res. 124, 185-214.

Bolle, O., Charlier, B., Bascou, J., Diot, H., McEnroe, S.A., 2014. Anisotropy of magnetic susceptibility versus lattice- and shape-preferred orientation in the Lac Tio hemoilmenite ore body (Grenville province, Quebec). Tectonophysics 629, 87-108.

Bourret, W., 1949. Aeromagnetic survey of the Allard Lake district, Quebec. Econ. Geol. 44, $732-740$.

Brown, L.L., McEnroe, S.A., Peck, W.H., Nilsson, L.P., 2011. Anorthosites as sources of magnetic anomalies. In: Petrovský, E., Herrero-Bervera, E., Harinarayana, T., Ivers, D. (Eds.), The Earth's Magnetic Interior. IAGA Special Sopron Book Series 1, pp. 321-342.

Buddington, A.F., Lindsley, D.H., 1964. Iron-titanium oxide minerals and synthetic equivalents. J. Petrol. 5, 310-357.

Bybee, G.M., Ashwal, L.D., Shirey, S.B., Horan, M., Mock, T., Andersen, T.B., 2014. Pyroxene megacrysts in Proterozoic anorthosites: implications for tectonic setting, magma source and magmatic processes at the Moho. Earth Planet. Sci. Lett. 389, 74-85.

Campbell, I.H., Roeder, P.L., Dixon, J.M., 1978. Plagioclase buoyancy in basaltic liquids as determined with a centrifuge furnace. Contrib. Mineral. Petrol. 67, 369-377.

Carmichael, I.S.E., 1961. The magnetic properties of ilmenite-heamatite crystals. Proc. R. Soc. A 263, 508-530.

Casquet, C., Pankhurst, R.J., Rapela, C.W., Galindo, C., Dahlquist, J., Baldo, E., Saavedra, J., González Casado, J.M., Fanning, C.M., 2005. Grenvillian massif-type anorthosites in the Sierras Pampeanas. J. Geol. Soc. 162, 9-12.

Cawthorn, R.G., Molyneux, T.G., 1986. Vanadiferous magnetite deposits of the Bushveld Complex. In: Anhaeusser, C.R., Maske, S. (Eds.), Mineral Deposits of Southern Africa. Geol Soc S Afr, pp. 1251-1266.

Charlier, B., Grove, T.L., 2012. Experiments on liquid immiscibility along tholeiitic liquid lines of descent. Contrib. Mineral. Petrol. 164 (1), 27-44.

Charlier, B., Duchesne, J.-C., Vander Auwera, J., 2006. Magma chamber processes in the Tellnes ilmenite deposit (Rogaland Anorthosite Province, SW Norway) and the formation of Fe-Ti ores in massif-type anorthosites. Chem. Geol. 234, 264-290.

Charlier, B., Skår, Ø., Korneliussen, A., Duchesne, J.-C., Vander Auwera, J., 2007. Ilmenite composition in the Tellnes Fe-Ti deposit, SW Norway: fractional crystallization, postcumulus evolution and ilmenite-zircon relation. Contrib. Mineral. Petrol. 154, 119-134.

Charlier, B., Sakoma, E., Sauvé, M., Stanaway, K., Vander Auwera, J., Duchesne, J.-C., 2008. The Grader layered intrusion (Havre-Saint-Pierre Anorthosite, Quebec) and genesis of nelsonite and other Fe-Ti-P ores. Lithos 101, 359-378.

Charlier, B., Namur, O., Duchesne, J.C., Wiszniewska, J., Parecki, A., Vander Auwera, J., 2009. Cumulate origin and polybaric crystallization of Fe-Ti oxide ores in the Suwalki anorthosite, northeastern Poland. Econ. Geol. 104, 205-221.

Charlier, B., Duchesne, J.C., Vander Auwera, J., Storme, J.Y., Maquil, R., Longhi, J., 2010a. Polybaric fractional crystallization of high-alumina basalt parental magmas in the Egersund-Ogna massif-type anorthosite (Rogaland, SW Norway) constrained by plagioclase and high-alumina orthopyroxene megacrysts. J. Petrol. 51, 2515-2546.

Charlier, B., Namur, O., Malpas, S., de Marneffe, C., Duchesne, J.C., Vander Auwera, J., Bolle, O., 2010b. Origin of the giant Allard Lake ilmenite ore deposit (Canada) by fractional crystallization, multiple magma pulses and mixing. Lithos 117, 119-134.

Charlier, B., Namur, O., Grove, T.L., 2013. Compositional and kinetic controls on liquid immiscibility in ferrobasalt-rhyolite volcanic and plutonic series. Geochim. Cosmochim. Acta 113, 79-93.

Chatterjee, N., Crowley, J.L., Mukherjee, A., Das, S., 2008. Geochronology of the 983-Ma Chilka Lake Anorthosite, Eastern Ghats Belt, India: implications for pre-Gondwana tectonics. J. Geol. 116, 105-118.
Chen, W., Zhou, M.-F., Zhao, T.-P., 2013. Differentiation of nelsonitic magmas in the formation of the 1.74 Ga Damiao Fe-Ti-P ore deposit, North China. Contrib. Mineral. Petrol. 165, 1341-1362.

Chernet, T., 1999. Effect of mineralogy and texture in the $\mathrm{TiO}_{2}$ pigment production process of the Tellnes ilmenite concentrate. Mineral. Petrol. 67, 21-32.

Clifford, T.N., Barton, E.S., Stern, R.A., Duchesne, J.-C., 2004. U-Pb zircon calendar for Namaquan (Grenville) crustal events in the granulite-facies terrane of the O'okiep copper district of South Africa. J. Petrol. 45, 669-691.

Conradie, J.A., Schoch, A.E., 1986. Iron-titanium oxide equilibria in copper-bearing diorites, Namaqualand. Trans. Geol. Soc. S. Afr. 89, 29-34.

Corfu, F., 2004. U-Pb age, setting and tectonic significance of the anorthosite-mangeritecharnockite-granite suite, Lofoten-Vesterålen, Norway. J. Petrol. 45, 1799-1819.

Corrigan, D., Hanmer, S., 1997. Anorthosites and related granitoids in the Grenville orogen: a product of convective thinning of the lithosphere? Geology 25, 61-64.

Corrigan, D., van Breemen, O., 1997. U-Pb age constraints for the lithotectonic evolution of the Grenville Province along the Mauricie transect, Quebec. Rev. Can. Sci. Terre 34, 299-316.

Corriveau, L., Perreault, S., Davidson, A., 2007. Prospective metallogenic settings of the Grenville Province. In: Goodfellow, W.D. (Ed.), Mineral Deposits of Canada: A Synthesis of Major Deposit-Types, District Metallogeny, The Evolution of Geological Provinces, and Exploration Methods. Geological Survey of Canada, Mineral Deposits Division, Special Publication, pp. 819-847.

Delano, J.W., 1980. Chemistry and liquidus phase relations of Apollo 15 red glass implications for the deep lunar interior. Proc. Lunar and Planetary Science Conference 11th 1 pp. 251-288.

Demaiffe, D., Hertogen, J., 1981. Rare earth element geochemistry and strontium isotopic composition of a massif-type anorthositic-charnockitic body: the Hidra massif (Rogaland, SW Norway). Geochim. Cosmochim. Acta 45, 1545-1561.

Demaiffe, D., Weis, D., Michot, J., Duchesne, J.C., 1986. Isotopic constraints on the genesis of the anorthosite suite of rocks. Chem. Geol. 57, 167-179.

Diot, H., Bolle, O., Lambert, J.-M., Launeau, P., Duchesne, J.C., 2003. The Tellnes ilmenite deposit (Rogaland, South Norway): magnetic and petrofabric evidence for emplacement of a Ti-enriched noritic crystal mush in a fracture zone. J. Struct. Geol. 25, 481-501.

Dobmeier, C., 2006. Emplacement of Proterozoic massif-type anorthosite during regional shortening: evidence from the Bolangir anorthosite complex (Eastern Ghats Province, India). Int. J. Earth Sci. 95, 543-555.

Dobmeier, C., Simmat, R., 2002. Post-Grenvillean transpression in the Chilka Lake area, Eastern Ghats Belt - implications for the geological evolution of peninsular India. Precambrian Res. 113, 243-268.

Doig, R., 1991. U-Pb zircon ages of Morin massif anorthosite suite rocks, Grenville province, Quebec. J. Geol. 99, 729-738.

Drüppel, K., Elsäßer, L., Brandt, S., Gerdes, A., 2012. Sveconorwegian mid-crustal ultrahigh-temperature metamorphism in Rogaland, Norway: U-Pb LA-ICP-MS geochronology and pseudosections of sapphirine granulites and associated paragneisses. J. Petrol. 54 (2), 305-350.

Duchesne, J.C., 1969. Les minerais noirs dans le massif magmatique stratiforme de Bjerkrem-Sogndal (Rogaland) et leur évolution dans la différenciation. PhD Thesis, University of Liège, 206 p.

Duchesne, J.C., 1970. Microstructures of Fe-Ti oxide minerals in the South Rogaland anorthositic complex (Norway). Ann. Soc. Geol. Belg. 93, 527-544.

Duchesne, J.C., 1972. Iron-titanium oxide minerals in the Bjerkrem-Sogndal Massif, South-western Norway. J. Petrol. 13, 57-81.

Duchesne, J.C., 1973. Les gisements d'oxides de Fe et Ti dans les roches anorthositiques du Rogaland (Norvège méridionale). In: Masson (Ed.), Les roches plutoniques dans leur rapport avec les gîtes minéraux, Paris, pp. 241-248.

Duchesne, J.C., 1996. Liquid ilmenite or liquidus ilmenite: a comment on the nature of ilmenite vein deposits. In: Demaiffe, D. (Ed.), Petrology and Geochemistry of Magmatic Suites of Rocks in the Continental and Oceanic Crusts. A Volume Dedicated to Professor Jean MichotRoyal Museum for Central Africa (Tervuren). Université Libre de Bruxelles, pp. 73-82.

Duchesne, J.C., 1999. Fe-Ti deposits in Rogaland anorthosites (South Norway): geochemical characteristics and problems of interpretation. Mineral. Deposita 34, 182-198.

Duchesne, J.C., Demaiffe, D., 1978. Trace elements and anorthosite genesis. Earth Planet Sci. Lett. 38, 249-272.

Duchesne, J.C., Hertogen, J., 1988. Le magma parental du lopolithe de Bjerkreim-Sokndal (Norvège méridionale). C. R. Acad. Sci. Paris 306, 45-48.

Duchesne, J.C., Wilmart, E., 1997. Igneous charnockites and related rocks from the Bjerkreim-Sokndal layered intrusion (Southwest Norway): a jotunite (hypersthene monzodiorite)-derived A-type granitoid suite. J. Petrol. 38, 337-369.

Duchesne, J.-C., Korneliussen, A., 2003. Ilmenite deposits and their geological environment. Norges Geol. Unders. Spec. Publ. 9, 134.

Duchesne, J.C., Roelandts, I., Demaiffe, D., Hertogen, J., Gijbels, R., De Winter, J., 1974 Rare-Earth data on monzonoritic rocks related to anorthosites and their bearing on the nature of the parental magma of the anorthositic series. Earth Planet. Sci. Lett. 24 325-335.

Duchesne, J.C., Charlier, B., 2005. Geochemistry of cumulates from the Bjerkreim-Soknda layered intrusion (S. Norway). Part I: constraints from major elements on the mechanism of cumulate formation and on the jotunite liquid line of descent. Lithos 83, 229-254.

Duchesne, J.C., Maquil, R., Demaiffe, D., 1985a. The Rogaland anorthosites: facts and speculations. In: Tobi, A.C., Touret, J.L.R. (Eds.), The Deep Proterozoic Crust in the North Atlantic Province. NATO Adv. Stud. Inst, Dordrecht, pp. 449-476.

Duchesne, J.C., Roelandts, I., Demaiffe, D., Weis, D., 1985b. Petrogenesis of monzonoritic dykes in the Egersund-Ogna anorthosite (Rogaland, S.W. Norway): trace elements and isotopic (Sr, Pb) constraints. Contrib. Mineral. Petrol. 90, 214-225. 
Duchesne, J.C., Denoiseux, B., Hertogen, J., 1987. The norite-mangerite relationships in the Bjerkreim-Sokndal layered lopolith (southwest Norway). Lithos 20, 1-17.

Duchesne, J.C., Liégeois, J.P., Vander Auwera, J., Longhi, J., 1999. The crustal tongue melting model and the origin of massive anorthosites. Terra Nova 11, 100-105.

Duchesne, J.C., Shumlyanskyy, L., Charlier, B., 2006. The Fedorivka layered intrusion (Korosten Pluton, Ukraine): an example of highly differentiated ferrobasaltic evolution. Lithos 89, 353-376.

Duchesne, J.-C., Vander Auwera, J., Liegeois, J.-P., Barton, E.S., Clifford, T.N., 2007. Geochemical constraints of the petrogenesis of the O'okiep Koperberg Suite and granitic plutons in Namaqualand, South Africa: a crustal source in Namaquan (Grenville) times. Precambrian Res. 153, 116-142.

Duchesne, J.C., Martin, H., Baginski, B., Wiszniewska, J., Vander Auwera, J., 2010. The origin of ferroan-potassic A-type granitoids: the case of the hornblende-biotite granite suite of the Mesoproterozoic Mazury complex, northeastern Poland. Can. Mineral. 48, 947-968.

Dymek, R.F., 1984. Sapphirine of possible igneous origin from the St-Urbain anorthosite massif, Quebec. Eos 65 (16), 295

Dymek, R.F., Owens, B.E., 2001. Petrogenesis of apatite-rich rocks (nelsonites and oxideapatite gabbronorites) associated with massif anorthosites. Econ. Geol. 96, 797-815.

Emslie, R.F., 1975. Pyroxene megacrysts from anorthositic rocks: a new clue to the sources and evolution of the parent magmas. Can. Mineral. 13, 138-145.

Emslie, R.F., 1978. Anorthosite massifs, rapakivi granites and late Proterozoic rifting of North America. Precambrian Res. 7, 61-98.

Emslie, R.F., 1980. Geology and petrology of the Harp Lake Complex, central Labrador: an example of Elsonian magmatism. Geol. Surv. Can. Bull. 293, 1-136.

Emslie, R.F., 1985. Proterozoic anorthosite massifs. In: Tobi, A.C., Touret, J.L.R. (Eds.), The Deep Proterozoic Crust in the North Atlantic Provinces. Reidel, Dordrecht, pp. 39-60.

Emslie, R.F., Hamilton, M.A., Theriault, R.J., 1994. Petrogenesis of a Midproterozoic anorthosite-mangerite-charnockite-granite (AMCG) complex - isotopic an chemical evidence from the Nain Plutonic Suite. J. Geol. 120, 539-558.

Force, E.R., 1991. Geology of titanium-mineral deposits. US Geological Survey Special Paper, p. 112.

Fram, M., Longhi, J., 1992. Phase equilibria of dikes associated with Proterozoic anorthosite complexes. Am. Mineral. 77, 605-616.

Francis, D., Scowen, P., Panneton, G., Doig, R., 2000. Contrasting Si-saturation in troctoliteanorthosite intrusions along the Manicouagan corridor of the Abitibi-Grenville transect. Can. J. Earth Sci. 37, 271-289.

Frost, C.D., Frost, B.R., 2011. On ferroan (A-type) granitoids: their compositional variability and modes of origin. J. Petrol. 52, 39-53.

Frost, C.D., Frost, B.R., 2013. Proterozoic ferroan feldspathic magmatism. Precambrian Res. $228,151-163$

Frost, B.R., Lindsley, D.H., 1992. Equilibria among Fe-Ti oxides, pyroxenes, olivine, and quartz: part II. Application. Am. Mineral. 77, 1004-1020.

Frost, B.R., Simons, J.P., 1991. Fe-Ti oxide deposits of the Laramie anorthosite complex: their geology and proposed economic utilization. In: Frost, B.R., Roberts, S. (Eds.) Wyoming Geological Association 42nd Annual Field Conference Guidebook, pp. 41-48.

Frost, B.R., Lindsley, D.H., Andersen, D.J., 1988. Fe-Ti oxide-silicate equilibria: assemblages with fayalitic olivine. Am. Mineral. 73, 727-740.

Gambogi, J., 2010. Titanium, U.S. Geological Survey. Minerals Yearbook 2008, p. 16.

Gibson, R.L., Robb, L.J., Kisters, A.F.M., Cawthorn, R.G., 1996. Regional setting and geological evolution of the Okiep Copper District, Namaqualand, South Africa. S. Afr. J. Geol. 99 107-120.

Gierth, E., Krause, H., 1973. Die ilmenitlagerstätte Tellnes (Süd-Norwegen). Nor. Geol. Tidsskr. 53, 359-402.

Gleissner, P., Drüppel, K., Taubald, H., 2010. Magmatic evolution of anorthosites of the Kunene intrusive complex, NW Namibia: evidence from oxygen isotope data and trace element zoning. J. Petrol. 51, 897-919.

Gleissner, P., Drüppel, K., Romer, R.L., 2011. The role of crustal contamination in massiftype anorthosites, new evidence from $\mathrm{Sr}-\mathrm{Nd}-\mathrm{Pb}$ isotopic composition of the Kunene Intrusive Complex, NW Namibia. Precambrian Res. 185, 18-36.

Gross, S.O., 1968. Titaniferous ores of the Lake Sanford district, New York. In: Ridge, J.D. (Ed.), Ore Deposits of the United States. Am. Inst. Mining Metall. Petrol. Eng, New York, pp. 140-153.

Gross, G., 1996. Mafic intrusion-hosted titanium-iron. In: Eckstrand, O.R, Sinclair, W.D. Thorpe, R.I. (Eds.), Geology of Canadian Mineral Deposit Types. Geologica Survey of Canada, Geology of Canada, pp. 573-582.

Grove, T.L., Baker, M.B., 1984. Phase equilibrium controls on the tholeiitic versus calcalkaline differentiation trends. J. Geophys. Res. 89, 3253-3274.

Gursky, D., Nechaev, S., Bobrov, A., 2003. Titanium deposits in Ukraine focused on the Proterozoic anorthosite-hosted massifs. In: Duchesne, J.C., Korneliussen, A. (Eds.) Ilmenite Deposits and Their Geological Environment. Norges Geol. Unders. Spec. Publ., pp. 21-26.

Haggerty, S.E., 1991. Oxide textures - A mini-atlas. In: Lindsley, D.H. (Ed.), Oxide Minerals: Petrologic and Magnetic Significance. Mineralogical Society of America, Reviews in Mineralogy 25, pp. 129-219.

Hammond, P., 1952. Allard Lake ilmenite deposits. Econ. Geol. 47, 634-649.

Hannah, J.L., Stein, H.J., 2002. Re-Os model for the origin of sulfide occurrences in Proterozoic anorthosites complex. Econ. Geol. 97, 371-383.

Hargraves, R.B., 1959. Magnetic anisotropy and remanent magnetization in hemoilmenite from ore deposits of Allard Lake, Quebec. J. Geophys. Res. 64, 1565-1573.

Hébert, C., Cadieux, A.-M., van Breemen, O., 2005. Temporal evolution and nature of TiFe-P mineralization in the anorthosite-mangerite-charnockite-granite (AMCG) suites of the south-central Grenville Province, Saguenay-Lac St. Jean area, Quebec, Canada. Can. J. Earth Sci. 42, 1865-1880.

Higgins, M.D., 1998. Origin of anorthosite by textural coarsening: quantitative measurements of a natural sequence of textural development. J. Petrol. 39, 1307-1323.
Higgins, M.D., van Breemen, O., 1996. Three generations of anorthosite-mangeritecharnockite-granite magmatism, contact metamorphism and tectonism in the Saguenay-Lac-St-Jean region, Grenville Province, Canada. Precambrian Res. 79, 347-362.

Hill, R., Roeder, P.L., 1973. The crystallization of spinel from basaltic liquid as a function of oxygen fugacity. J. Geol. 82, 709-729.

Irvine, T.N., 1977. Origin of chromitite layers in the Muskox intrusion and other stratiform intrusions: a new interpretation. Geology 5, 273-277.

Janardhan, A.S., Wiebe, R.A., 1985. Petrology and geochemistry of the Oddanchatram anorthosite and associated basic granulites, Tamil Nadu, South India. J. Geol. Soc. India 26, 163-176.

Jansen, J.B.H., Blok, R.J.P., Bos, A., Scheelings, M., 1985. Geothermometry and geobarometry in Rogaland and preliminary results from the Bamble area. In: Tobi, A.C., Touret, J.L.R. (Eds.), The Deep Proterozoic Crust in the North Atlantic Provinces. Reidel, Dordrecht, pp. 499-516.

Jensen, J.C., Nielsen, F.M., Duchesne, J.C., Demaiffe, D., Wilson, J.R., 1993. Magma influx and mixing in the Bjerkreim-Sokndal layered intrusion, South Norway: evidence from the boundary between two megacyclic units at Storeknuten. Lithos 29, 311-325.

Juster, T.C., Grove, T.L., Perfit, M.R., 1989. Experimental constraints on the generation of FeTi basalts, andesites, and rhyodacites at the Galapagos spreading center, $85^{\circ} \mathrm{W}$ and $95^{\circ}$ W. J. Geophys. Res. 94, 9251-9274.

Keppie, J.D., Dostal, J., Cameron, K.L., Solari, L.A., Ortega-Gutierrez, F., Lopez, R., 2003. Geochronology and geochemistry of Grenvillian igneous suites in the northern Oaxacan Complex, southern Mexico: tectonic implications. Precambrian Res. 120, 365-389.

Kohlstedt, D.L., Zimmerman, M.E., Mackwell, S.J., 2010. Stress-driven melt segregation in partially molten feldspathic rocks. J. Petrol. 51, 9-19.

Kolker, A., 1982. Mineralogy and geochemistry of Fe-Ti oxide and apatite (nelsonite) deposits and evaluation of the liquid immiscibility hypothesis. Econ. Geol. 77, $1146-1158$

Krause, H., Pape, H., 1977. Untersuchungen zum geologischen und petrographischen aufbau des Storgangen-ilmeniterzkörpers und seiner nebengesteinseinheiten (Süd-Norwegen). Nor. Geol. Tidsskr. 57, 263-284.

Krause, H., Zeino-Mahmalat, R., 1970. Untersuchungen an erz und nebengestein der grube Blåfjell in SW-Norwegen. Nor. Geol. Tidsskr. 50, 45-88.

Krause, H., Gierth, E., Schott, W., 1985. Ti-Fe deposits in the South Rogaland igneous complex, especially in the anorthosite-massif of Åna-Sira. Nor. Geol. Unders. 402, $25-37$.

Krause, O., Dobmeier, C., Raith, M.M., Mezger, K., 2001. Age of emplacement of massiftype anorthosites in the Eastern Ghats Belt, India: constraints from U-Pb zircon dating and structural studies. Precambrian Res. 109, 25-38.

Kushiro, I., 1980. Viscosity, density and structure of silicate melts at high pressures, and their petrological applications. In: Hargraves, R.B. (Ed.), Physics of Magmatic Processes. Princeton University Press, Princeton, pp. 93-120.

Kushiro, I., Fujii, T., 1977. Floatation of plagioclase in magma at high pressures and its bearing on the origin of anorthosite. Proc. Jpn. Acad. Ser. B 53, 262-266.

Lafrance, B., John, B.E., Scoates, J.S., 1996. Syn-emplacement recrystallization and deformation microstructures in the Poe Mountain anorthosite, Wyoming Contrib. Mineral. Petrol. 122, 431-440.

Lattard, D., Sauerzapf, U., Käsemann, M., 2005. New calibration data for the Fe-Ti oxide thermo-oxybarometers from experiments in the Fe-Ti-O system at 1 bar, 1,000$1,300{ }^{\circ} \mathrm{C}$ and a large range of oxygen fugacities. Contrib. Mineral. Petrol. 149, 735-754.

Li, C., Lightfoot, P.C., Amelin, Y., Naldrett, A.J., 2000. Contrasting petrological and geochemical relationships in the Voisey's Bay and Mushuau intrusions, Labrador, Canada: implications for ore genesis. Econ. Geol. 95, 771-799.

Li, L., Li, H., Chen, Z., Wang, D., Chen, W., 2010. Hydrothermal mineralization and fluid inclusion study on the Heishan iron deposit, Chengde County, Hebei Province, China. Acta Petrol. Sin. 26, 858-870.

Li, H., Li, L., Zhang, Z., Santosh, M., Liu, M., Cui, Y., Yang, X., Chen, J., Yao, T., 2014. Alteration of the Damiao anorthosite complex in the northern North China Craton: implications for high-grade iron mineralization. Ore Geol. Rev. 57, 574-588.

Lindsley, D.H., 1981. Some experiments pertaining to the magnetite ulvöspinel miscibility gap. Am. Mineral. 66, 759-762.

Lindsley, D.H., 1991. Experimental studies of oxide minerals. In: Lindsley, D.H. (Ed.) Oxide Minerals: Petrologic and Magnetic Significance. Mineralogical Society of America, Reviews in Mineraly 25, pp. 69-106

Lindsley, D.H., 2003. Do Fe-Ti oxide magmas exist? Geology: yes; Experiments: no! Norges Geol. Unders. Spec. Publ. 9, 34-35.

Lindsley, D.H., Frost, B.R., 1992. Equilibria among Fe-Ti oxides, pyroxenes, olivine, and quartz. 1. Theory. Am. Mineral. 77, 987-1003.

Lindsley, D.H., Epler, N.A., Bolsover, LR., 1988. Nature and Origin of the Sybille Fe-Ti Oxide Deposit, Laramie Anorthosite Complex, Wyoming, Penrose Conference: The Origin and Evolution of Anorthosites and Associated Rock (14-19 August 1988, Wyoming)

Lindsley, D.H., Frost, B.R., Frost, C.D., Scoates, J.S., 2010. Petrology, geochemistry, and structure of the Chugwater Anorthosite, Laramie Anorthosite Complex, Southeastern Wyoming. Can. Mineral. 48, 887-923.

Lister, G.F., 1966. The composition and origin of selected iron-titanium deposits. Econ. Geol. 61, 275-310.

Longhi, J., 2005. A mantle or mafic crustal source for Proterozoic anorthosites? Lithos 83, 183-198.

Longhi, J., Vander Auwera, J., Fram, M., Monthieth, J.N., 1993. Pressure effects, kinetics and rheology of anorthositic and related magmas. Am. Mineral. 78, 1016-1030.

Longhi, J., Vander Auwera, J., Fram, M.S., Duchesne, J.C., 1999. Some phase equilibrium constraints on the origin of Proterozoic (massif) anorthosites and related rocks. J. Petrol. 40, 339-362. 
Maier, W.D., Barnes, S.-J., 1996. Unusually high concentrations of magnetite at Caraiba and other Cu-sulfide deposits in the Curaca valley, Bahia, Brazil. Can. Mineral. 34, 717-731.

Maier, W.D., Barnes, S.-J., 1999. The origin of Cu sulfide deposits in the Curaca Valley, Bahia, Brazil: evidence from $\mathrm{Cu}, \mathrm{Ni}$, Se and platinum-group element concentrations. Econ. Geol. 94, 165-183.

Maier, W.D., Rasmussen, B., Fletcher, I.R., Li, C., Barnes, S.-J., Huhma, H., 2013. The Kunene anorthosite complex, Namibia, and its satellite intrusions: geochemistry, geochronology, and economic potential. Econ. Geol. 108, 953-986.

Markl, G., Frost, B.R., 1999. The origin of anorthosites and related rocks from the Lofoten islands, northern Norway: II. Calculation of parental liquid compositions for anorthosites. J. Petrol. 40, 61-77.

Markl, G., Frost, B.R., Bucher, K., 1998. The origin of anorthosites and related rocks from the Lofoten islands, northern Norway: I. Field relations and estimation of intrinsic variables. J. Petrol. 39, 1425-1452.

Martignole, J., Schrijver, K., 1970. Tectonic significance and evolution of the Morin anorthosite, Grenville Province, Quebec. Bull. Geol. Soc. Finl. 42, 165-209.

Martignole, J., Machado, N., Nantel, S., 1993. Timing of intrusion and deformation of the Riviere-Pentecote anorthosite (Grenville Province). J. Geol. 101, 652-658.

Mayer, A., Hofmann, A.W., Sinigoi, S., Morais, E., 2004. Mesoproterozoic Sm-Nd and U-Pb ages for the Kunene Anorthosite Complex of SW Angola. Precambrian Res. 133, 187-206.

McEnroe, S.A., Robinson, P., Panish, P.T., 1996. Rock-magnetic properties, oxide mineralogy, and mineral chemistry in relation to aeromagnetic interpretation and the search of ilmenite reserves. Norges Geologiske Undersøkelse Report 96.060, p. 148.

McEnroe, S.A., Robinson, P., Panish, P.T., 2001. Aeromagnetic anomalies, magnetic petrology, and rock magnetism of hemo-ilmenite- and magnetite-rich cumulate rocks from the Sokndal Region, South Rogaland, Norway. Am. Mineral. 86, 1447-1468.

McEnroe, S.A., Harrison, R.J., Robinson, P., Langenhorst, F., 2002. Nanoscale haematiteilmenite lamellae in massive ilmenite rock: an example of 'lamellar magnetism' with implications for planetary magnetic anomalies. Geophys. J. Int. 151, 890-912.

McEnroe, S.A., Langenhorst, F., Robinson, P., Bromiley, G.D., Shaw, C.S.J., 2004. What is magnetic in the lower crust? Earth Planet. Sci. Lett. 226, 175-192.

McEnroe, S.A., Robinson, P., Langenhorst, F., Frandsen, C., Terry, M.P., Boffa Ballaran, T., 2007. Magnetization of exsolution intergrowths of hematite and ilmenite: mineral chemistry, phase relations, and magnetic properties of hemo-ilmenite ores with micron- to nanometer-scale lamellae from Allard Lake, Quebec. J. Geophys. Res. 112, B10103.

McEnroe, S.A., Brown, L.L., Robinson, P., 2009. Remanent and induced magnetic anomalies over a layered intrusion: effects from crystal fractionation and magma recharge. Tectonophysics 478, 119-134.

McLelland, J., Ashwal, L., Moore, L., 1994. Composition and petrogenesis of oxide-, apatiterich gabbronorites associated with Proterozoic anorthosite massifs: examples from the Adirondack Mountains, New York. Contrib. Mineral. Petrol. 116, 225-238.

McLelland, J.M., Bickford, M.E., Hill, B.M., Clechenko, C.C., Valley, J.W., Hamilton, M.A., 2004. Direct dating of Adirondack massif anorthosite by U-Pb SHRIMP analysis of igneous zircon: implications for AMCG complexes. Geol. Soc. Am. Bull. 116, 1299-1317.

McLelland, J.M., Selleck, B.W., Hamilton, M.A., Bickford, M.E., 2010. Late- to post-tectonic setting of some major Proterozoic anorthosite-mangerite-charnockite-granite (AMCG) suites. Can. Mineral. 48, 729-750.

Meyer, G.B., Schiellerup, H., Tegner, C., 2002. Chemical characterisation of ilmenite, magnetite and apatite in the Bjerkreim-Sokndal Layered Intrusion, Rogaland, South Norway. Nor. Geol. Unders. Rep. 2002 (042), 1-18.

Mitchell, J.N., Scoates, J.S., Frost, C.D., 1995. High-Al gabbros in the laramie anorthosite complex, Wyoming - implications for the composition of melts parental to Proterozoic anorthosite. Contrib. Mineral. Petrol. 119, 166-180.

Mitchell, J.N., Scoates, J.S., Frost, C.D., Kolker, A., 1996. The geochemical evolution of anorthosite residual magmas in the Laramie Anorthosite Complex, Wyoming. J. Petrol. 37, 637-660.

Möller, A., O'Brien, P.J., Kennedy, A., Kröner, A., 2003. Linking growth episodes of zircon and metamorphic textures to zircon chemistry: an example from the ultrahightemperature granulites of Rogaland (SW Norway). Geol. Soc. Lond., Spec. Publ. 220, 65-81.

Morgan, J.W., Stein, H.J., Hannah, J.L., Markey, R.J., Wiszniewska, J., 2000. Re-Os study of Fe-Ti-V oxide and Fe-Cu-Ni sulfide deposits, Suwalki anorthosite massif, northeast Poland. Mineral. Deposita 35, 391-401.

Morisset, C.E., Scoates, J.S., 2008. Origin of zircon rims around ilmenite in mafic plutonic rocks of Proterozoic anorthosite suites. Can. Mineral. 46, 289-304.

Morisset, C.-E., Scoates, J.S., Weis, D., Friedman, R.M., 2009. U-Pb and ${ }^{40} \mathrm{Ar} /{ }^{39} \mathrm{Ar}$ geochronology of the Saint-Urbain and Lac Allard (Havre-Saint-Pierre) anorthosites and their associated Fe-Ti oxide ores, Québec: evidence for emplacement and slow cooling during the collisional Ottawan orogeny in the Grenville Province. Precambrian Res. 174, 95-116.

Morisset, C.-E., Scoates, J.S., Weis, D., Sauve, M., Stanaway, K.J., 2010. Rutile-bearing ilmenite deposits associated with the Proterozoic Saint-Urbain and Lac Allard anorthosite massifs, Grenville Province, Québec. Can. Mineral. 48, 821-849.

Morisset, C.-E., Williamson, M.-C., Hipkin, V., 2013. Investigation of three Fe-Ti oxide deposits associated with Grenvillian anorthosite massifs as potential source for lunar analogue ilmenite. Can. J. Earth Sci. 50 (1), 64-77.

Morse, S.A., 1973. The feldspar/magma density paradox. In: Morse, S.A. (Ed.), The Nain Anorthosite Project, Labrador: Field Report 1972. University of Massachusetts at Amherst, Amherst, MA, pp. 113-116.

Morse, S.A., 1980. Kiglapait mineralogy II: Fe-Ti oxide minerals and the activities of oxygen and silica. J. Petrol. 21, 685-719.

Morse, S.A., 1982. A partisan review of Proterozoic anorthosites. Am. Mineral. 67 1087-1100.
Myers, J.S., Voordouw, R.J., Tettelaar, T.A., 2008. Proterozoic anorthosite-granite Nain batholith: structure and intrusion processes in an active lithosphere-scale fault zone, northern Labrador. Can. J. Earth Sci. 45, 909-934.

Namur, O., Charlier, B., Holness, M.B., 2012. Dual origin of Fe-Ti-P gabbros by immiscibility and fractional crystallization of evolved tholeiitic basalts in the Sept Iles layered intrusion. Lithos 154, 100-114.

Nasipuri, P., Bhattacharya, A., 2007. Melt-assisted interior to margin switch from dislocation to diffusion creep in coarse grained plagioclase: evidence from a deformed anorthosite pluton. J. Struct. Geol. 29, 1327-1338.

Naslund, H.R., 1987. Lamellae of baddeleyite and Fe-Cr-spinel in ilmenite from the Basistoppen sill, East Greenland. Can. Mineral. 25, 91-96.

Oliveira, E.P., Tarney, J., 1995. Genesis of the Precambrian copper-rich Caraiba hypersthenite-norite complex, Brazil. Mineral. Deposita 30, 351-373.

Olsen, K.E., Morse, S.A., 1990. Regional Al-Fe mafic magmas associated with anorthositebearing terranes. Nature 344, 760-762.

Owens, B.E., Dymek, R.F., 1992. Fe-Ti-P-rich rocks and massif anorthosite: problems of interpretation illustrated from the Labrieville and St-Urbain plutons, Quebec. Can. Mineral. 30, 163-190.

Owens, B.E., Dymek, R.F., 1999. A geochemical reconnaissance of the Roseland anorthosite complex, Virginia, and comparisons with andesine anorthosites from the Grenville Province, Quebec. In: Sinha, A.K. (Ed.), Basement Tectonics 13. Springer, pp. 217-232.

Owens, B.E., Rockow, M.W., Dymek, R.F., 1993. Jotunites from the Grenville Province, Quebec: petrological characteristics and implications for massif anorthosite petrogenesis. Lithos 30, 57-80.

Owens, B.E., Dymek, R.F., Tucker, R.D., Brannon, J.C., Podosek, F.A., 1994. Age and radiogenic isotopic composition of a late- to posttectonic anorthosite in the Grenville Province - the Labrieville massif, Quebec. Lithos 31, 189-206.

Paludan, J., Hansen, U.B., Olesen, N.Ø., 1994. Structural evolution of the Precambrian Bjerkreim-Sokndal intrusion, South Norway. Nor. Geol. Tidsskr. 74, 185-198.

Pang, K.-N., Zhou, M.-F., Qi, L., Shellnutt, G., Wang, C.Y., Zhao, D., 2010. Flood basalt-related Fe-Ti oxide deposits in the Emeishan large igneous province, SW China. Lithos 119 $123-136$.

Passchier, C.W., Trouw, R.A.J., 2005. Microtectonics. 2d ed. Springer-Verlag, Berlin, Heidelberg.

Perreault, S., 2003. Contrasting styles of Fe-Ti mineralization in the Havre-Saint-Pierre anorthosite suite, Quebec's North Shore, Canada. Norges Geol. Unders. Spec. Publ. 9, 87-90.

Perreault, S., Hébert, C., 2003. Review of Fe-Ti $\pm \mathrm{V}$ and $\mathrm{Fe}-\mathrm{Ti}-\mathrm{P}_{2} \mathrm{O}_{5} \pm \mathrm{V}$ deposits associated with anorthosite suites in the Grenville Province, Québec. Norges Geol. Unders. Spec. Publ. 9, 83-84.

Philpotts, A.R., 1967. Origin of certain iron-titanium oxide and apatite rocks. Econ. Geol 62, 303-315.

Philpotts, A.R., 1982. Compositions of immiscible liquids in volcanic rocks. Contrib. Mineral. Petrol. 80, 201-218.

Priesemann, F.-D., Krause, H., 1985. The Selvåg deposit: a Proterozoic magmatic Fe-Ti-V occurrence on Vesterålen, northern Norway. Nor. Geol. Unders. 402, 51-64.

Ramdohr, P., 1980. The Ore Minerals and Their Intergrowths. Pergamon Press, Oxford, UK 1207 p.

Rapp, J.F., Klemme, S., Butler, I.B., Harley, S.L., 2010. Extremely high solubility of rutile in chloride and fluoride-bearing metamorphic fluids: an experimental investigation. Geology 38, 323-326.

Reynolds, I., 1986. Vanadium-bearing titaniferous iron ores of the Rooiwater Complex north-eastern Transvaal. In: Anhaeusser, C.R., Maske, S. (Eds.), Mineral Deposits of Southern Africa. Geological Society of South Africa, Johannesburg, pp. 451-460.

Rivers, T., Corrigan, D., 2000. Convergent margin on southeastern Laurentia during the Mesoproterozoic: tectonic implications. Can. J. Earth Sci. 37, 359-383.

Rivers, T., Martignole, J., Gower, C.F., Davidson, A., 1989. New tectonic divisions of the Grenville Province, southeastern Canadian Shield. Tectonics 8, 63-84.

Robinson, P., Harrison, R.J., McEnroe, S.A., Hargraves, R.B., 2002. Lamellar magnetism in the haematite-ilmenite series as an explanation for strong remanent magnetization. Nature 418, 517-520.

Robinson, P., Kullerud, K., Tegner, C., Robins, B., McEnroe, S.A., 2003. Could the Tellnes ilmenite deposit have been produced by in-situ magma mixing? Norges Geol. Unders. Spec. Publ. 9, 107-108.

Robinson, P., Fabian, K., McEnroe, S.A., Heidelbach, F., 2013. Influence of lattice-preferred orientation with respect to magnetizing field on intensity of remanent magnetization in polycrystalline hemo-ilmenite. Geophys. J. Int. 192, 514-536.

Roedder, E., 1978. Silicate liquid immiscibility in magmas and in the system $\mathrm{K}_{2} \mathrm{O}-\mathrm{FeO}$ $\mathrm{Al}_{2} \mathrm{O}_{3}-\mathrm{SiO}_{2}$ : an example of serendipity. Geochim. Cosmochim. Acta 42, 1597-1617.

Romer, R.L., 1996. Contiguous Laurentia and Baltica before the Grenvillian-Sveconorwegian orogeny? Terra Nova 8, 173-181.

Rose, E.R., 1969. Geology of titanium and titaniferous deposits of Canada. Geol. Surv. Can. Econ. Geol. Rep. 25, 177.

Ryan, B., 2000. The Nain-Churchill boundary and the Nain Plutonic Suite: a regional perspective on the geologic setting of the Voisey's Bay Ni-Cu-Co deposit. Econ. Geol. 95, 703-724.

Sauerzapf, U., Lattard, D., Burchard, M., Engelmann, R., 2008. The titanomagnetiteilmenite equilibrium: new experimental data and thermo-oxybarometric application to the crystallization of basic to intermediate rocks. J. Petrol. 49, 1161-1185.

Schärer, U., Wilmart, E., Duchesne, J.C., 1996. The short duration and anorogenic character of anorthosite magmatism: U-Pb dating of the Rogaland complex, Norway. Earth Planet. Sci. Lett. 139, 335-350.

Schiellerup, H., Lambert, D.D., Prestvik, T., Robins, B., McBride, J.S., Larsen, R.B., 2000. Re-Os isotopic evidence for a lower crustal origin of massif-type anorthosites. Nature $405,781-784$ 
Schiellerup, H., Korneliussen, A., Heldal, T., Marker, M., Bjerkgård, T., Nilsson, L.-P., 2003. Mineral resources in the Rogaland Anorthosite Province, South Norway: origins, history and recent developments. Norges Geol. Unders. Spec. Publ. 9, 116-134.

Scoates, J.S., 2000. The plagioclase-magma density paradox re-examined and the crystallization of Proterozoic anorthosites. J. Petrol. 41, 627-649.

Scoates, J.S., Chamberlain, K.R., 1997. Orogenic to post-orogenic origin for the $1.76 \mathrm{Ga}$ Horse Creek anorthosite complex, Wyoming, USA. J. Geol. 105, 331-343.

Scoates, J.S., Chamberlain, K.R., 2003. Geochronologic, geochemical and isotopic constraints on the origin of monzonitic and related rocks in the Laramie anorthosite complex, Wyoming, USA. Precambrian Res. 124, 269-304.

Scoates, J.S., Frost, C.D., 1996. A strontium and neodymium isotopic investigation of the Laramie anorthosites, Wyoming, USA: implications for magma chamber processes and the evolution of magma conduits in Proterozoic anorthosites. Geochim. Cosmochim. Acta 60, 95-107.

Scoates, J.S., Lindsley, D.H., 2000. New insights from experiments on the origin of anorthosite. Eos 81, F1300.

Scoates, J.S., Frost, C.D., Mitchell, J.N., Lindsley, D.H., Frost, B.R., 1996. Residual-liquid origin for a monzonitic intrusion in a mid-Proterozoic anorthosite complex: the Sybille intrusion, Laramie anorthosite complex, Wyoming. GSA Bull. 108, 1357-1371.

Scoates, J.S., Lindsley, D.H., Frost, B.R., 2010. Magmatic and structural evolution of an anorthositic magma chamber: the Poe mountain intrusion, Laramie Anorthosite complex, Wyoming. Can. Mineral. 48, 851-885.

Seifert, K.E., Dymek, R.F., Whitney, P.R., Haskin, L.A., 2010. Geochemistry of massif anorthosite and associated rocks, Adirondack Mountains, New York. Geosphere 6, 855-899.

Skridlaite, G., Wiszniewska, J., Duchesne, J.-C., 2003. Ferro-potassic A-type granites and related rocks in NE Poland and S Lithuania: west of the East European Craton. Precambrian Res. 124, 305-326.

Snyder, D., Carmichael, I.S.E., Wiebe, R.A., 1993. Experimental study of liquid evolution in an Fe-rich, layered mafic intrusion: constrains of Fe-Ti oxide precipitation on the $\mathrm{T}-\mathrm{fO}_{2}$ and $\mathrm{T}-\rho$ paths of tholeiitic magmas. Contrib. Mineral. Petrol. 113, 73-86.

Spencer, K.J., Lindsley, D.H., 1981. A solution model for coexisting iron-titanium oxides, Am. Mineral. 66, 1189-1201.

Sun, J., Du, W., Wang, D., Li, J., Xu, G., Han, Y., 2009. Geological characteristic and genesis of the Heishan V-Ti magnetite deposit in Damiao, Chengde Hebei Province. Acta Geol. Sin. 83, 1344-1363.

Taylor, S.R., Campbell, I.H., McCulloch, M.T., McLennan, S.M., 1984. A lower crustal origin for massif-type anorthosites. Nature 311, 372-374.

Thy, P., 1982. Titanomagnetite and ilmenite in the Fongen-Hyllingen basic complex Norway. Lithos 15, 1-16

Toplis, M.J., Carroll, M.R., 1995. An experimental study of the influence of oxygen fugacity on Fe-Ti oxide stability, phase relations, and mineral-melt equilibria in ferro-basaltic systems. J. Petrol. 36, 1137-1170.

Toplis, M., Corgne, A., 2002. An experimental study of element partitioning between magnetite, clinopyroxene and iron-bearing silicate liquids with particular emphasis on vanadium. Contrib. Mineral. Petrol. 144, 22-37.
Toplis, M.J., Dingwell, D., Libourel, G., 1994a. The effect of phosphorous on the iron redox ratio, viscosity, and density of an evolved ferrobasalt. Contrib. Mineral. Petrol. 117, 293-304.

Vander Auwera, J., Longhi, J., 1994. Experimental study of a jotunite (hypersthene monzodiorite): constraints on the parent magma composition and crystallization conditions $\left(P, T, \mathrm{fO}_{2}\right)$ of the Bjerkreim-Sokndal layered intrusion (Norway). Contrib. Mineral. Petrol. 118, 60-78.

Vander Auwera, J., Longhi, J., Duchesne, J.C., 1998. A liquid line of descent of the jotunite (hypersthene monzodiorite) suite. J. Petrol. 39, 439-468.

Vander Auwera, J., Weis, D., Duchesne, J.C., 2006. Marginal mafic intrusions as indicators of downslope draining of dense residual melts in anorthositic diapirs? Lithos 89, 329-352.

Vander Auwera, J., Bolle, O., Bingen, B., Liégeois, J.P., Bogaerts, M., Duchesne, J.C., De Waele, B., Longhi, J., 2011. Sveconorwegian massif-type anorthosites and related granitoids result from post-collisional melting of a continental arc root. Earth Sci. Rev. 107, 375-397.

Vincent, E.A., 1960. Ulvöspinel in the Skaergaard intrusion. Greenland. Neues Jahrb. Mineral. Abh. 94, 993-1016.

Vincent, E.A., Phillips, R., 1954. Iron titanium oxide minerals in layered gabbros of the Skaergaard intrusion, East Greenland. Geochimica et Cosmochimica Acta 6, 1-26.

Warren, C.H., 1912. The ilmenite rock near St. Urbain: a new occurrence of rutile and sapphirine. Am. J. Sci. 33, 263-277.

Watson, T.L., Taber, S., 1910. Nelsonite, a new rock type; its occurrence, association, and composition. Geol. Soc. Am. Bull. 21, 787.

Westphal, M., Schumacher, J.C., Boschert, S., 2003. High-temperature metamorphism and the role of magmatic heat sources at the Rogaland Anorthosite Complex in Southwestern Norway. J. Petrol. 44, 1145-1162.

Wilmart, E., Demaiffe, D., Duchesne, J.C., 1989. Geochemical constraints on the genesis of the Tellnes ilmenite deposit, Southwest Norway. Econ. Geol. 84, 1047-1056.

Wilson, J.R., Robins, B., Nielsen, F.M., Duchesne, J.C., Vander Auwera, J., 1996. The Bjerkreim-Sokndal layered intrusion, Southwest Norway. In: Cawthorn, R.G. (Ed.), Layered Intrusions. Elsevier, Amsterdam, pp. 231-255.

Wiszniewska, J., Claesson, S., Stein, H.J., Vander Auwera, J., Duchesne, J.C., 2002. The northeastern Polish anorthosite massifs: petrological, geochemical and isotopic evidence for a crustal derivation. Terra Nova 14, 1-10.

Woodruff, L.G., Nicholson, S.W., Fey, D.L., 2013. A deposit model for magmatic irontitanium-oxide deposits related to Proterozoic massif anorthosite plutonic suites. U.S. Geological Survey Scientific Investigations Report 2013-5091 (47 pp.).

Xie, G., 1982. Petrological characteristics of the Damiao anorthosite complex in Hebei Province, China. Geochemistry 1, 369-386.

Zhang, S.-H., Liu, S.-W., Zhao, Y., Yang, J.-H., Song, B., Liu, X.-M., 2007. The 1.75-1.68 Ga anorthosite-mangerite-alkali granitoid-rapakivi granite suite from the northern North China Craton: magmatism related to a Paleoproterozoic orogen. Precambrian Res. 155, 287-312.

Zhao, T.-P., Chen, W., Zhou, M.-F., 2009. Geochemical and Nd-Hf isotopic constraints on the origin of the $\sim 1.74$-Ga Damiao anorthosite complex, North China Craton. Lithos $113,673-690$. 